\title{
THE FRASER-HORN AND APPLE PROPERTIES
}

\author{
JOEL BERMAN AND W. J. BLOK
}

\begin{abstract}
We consider varieties $\mathscr{V}$ in which finite direct products are skew-free and in which the congruence lattices of finite directly indecomposables have a unique coatom. We associate with $\mathscr{V}$ a family of derived varieties, $d(\mathscr{V})$ : a variety in $d(\mathscr{V})$ is generated by algebras $\mathbf{A}$ where the universe of $\mathbf{A}$ consists of a congruence class of the coatomic congruence of a finite directly indecomposable algebra $\mathbf{B} \in \mathscr{V}$ and the operations of $\mathbf{A}$ are those of $\mathbf{B}$ that preserve this congruence class. We also consider the prime variety of $\mathscr{V}$, denoted $\mathscr{V}_{0}$, generated by all finite simple algebras in $\mathscr{V}$. We show how the structure of finite algebras in $\mathscr{V}$ is determined to a considerable extent by $\mathscr{V}_{0}$ and $d(\mathscr{V})$. In particular, the free $\mathscr{V}$-algebra on $n$ generators, $\mathbf{F}_{\mathscr{V}}(n)$, has as many directly indecomposable factors as $\mathbf{F}_{\mathscr{V}_{0}}(n)$ and the structure of these factors is determined by the varieties $d(\mathscr{V})$. This allows us to produce in many cases explicit formulas for the cardinality of $\mathbf{F}_{\mathscr{V}}(n)$. Our work generalizes the structure theory of discriminator varieties and, more generally, that of arithmetical semisimple varieties. The paper contains many examples of algebraic systems that have been investigated in different contexts; we show how these all fit into a general scheme.
\end{abstract}

The literature on free algebras in varieties of algebras is quite extensive. Most of the work done in this area, however, concerns the structure or cardinality of the free algebras in specific varieties, while only a few attempts have been made to develop a more general theory which might explain the structure of free algebras in certain classes of varieties in more general terms. For example, Quackenbush [1974] did this for discriminator varieties, and Plonka for regular varieties [1971]. Recently, Cornish [1983], using a categorical approach, described the free algebras in varieties whose algebras admit an involution operator.

In the present paper we study a class of locally finite varieties whose finite free algebras are, to a considerable degree, determined by the finite free algebras in simpler varieties, naturally associated with the original ones. The class we have in mind consists of the varieties which have the Fraser-Horn Property (FHP for short) and what we call the Apple Property (AP for short).

A variety has the Fraser-Horn Property if there are no skew congruences on any direct product of a finite number of algebras in the variety. If the congruence lattice of an algebra $\mathbf{A}$ has a unique coatom-i.e., is isomorphic to $\mathbf{L} \oplus 1$, where $\mathbf{L}$ is a lattice with 0,1 -then clearly $\mathbf{A}$ is directly indecomposable. A variety $\mathscr{V}$ has the Apple Property if the converse holds as well for all finite algebras; that is, if the finite

Received by the editors October 7, 1985.

1980 Mathematics Subject Classification. Primary 08A05, 08B20, 08A40; Secondary 03G25, 06D20.

Key words and phrases. Free algebra, Fraser-Horn Property, arithmetical variety, semisimple algebra, directly indecomposable, pseudo-complemented lattice, congruence lattice, clone of operations. 
directly indecomposables in $\mathscr{V}$ are precisely the finite algebras whose congruence lattices have a unique coatom. If a variety $\mathscr{V}$ has FHP and the Apple Property we say $\mathscr{V}$ has FHAP.

The first variety to be associated with a locally finite variety $\mathscr{V}$ with FHAP is its prime variety $\mathscr{V}_{0}$, the variety generated by the finite simple algebras in $\mathscr{V}$. Under an additional (weak) assumption on $\mathscr{V}$ it turns out $\mathscr{V}_{0}$ is in fact an arithmetical semisimple variety. In this case the finite free algebras in $\mathscr{V}_{0}$ are direct products of simple algebras, and we show that the number of direct factors of the free $n$-generated algebra in $\mathscr{V}$ equals that of the free $n$-generated algebra in $\mathscr{V}_{0}$. We call the indecomposable factors of the finitely generated free algebras in $\mathscr{V}$ the free directly indecomposable algebras in $\mathscr{V}$; they are free in a certain subcategory of $\mathscr{V}$. Since $\mathscr{V}$ has the Apple Property the congruence lattice of such an indecomposable $\mathbf{B}$ has a unique coatom $\alpha$. The congruence classes of $\alpha$ can be considered as algebras in their own right. These algebras give rise to a family of varieties, the derived varieties of $\mathscr{V}$. The focus of this paper is the relationship between locally finite varieties $\mathscr{V}$ with FHAP and their prime and derived varieties, in particular regarding their respective finite free algebras.

In $\$ 2$ we formally introduce and discuss the Fraser-Horn and Apple Properties. In $\$ 3$ we investigate the structure of the congruence lattices of the algbras in a locally finite variety with FHAP and characterize them. One of their main features is that they are dually pseudo-complemented. Interestingly, any finite algebra $\mathbf{A}$ with the property that its simple homomorphic images generate an arithmetical variety has a dually pseudo-complemented congruence lattice (Theorem 3.6).

In $\$ 4$ we define the prime variety and formulate conditions which guarantee it to be arithmetical and semisimple. We show that if $\mathscr{V}$ has FHAP and is generated by a finite algebra $\mathbf{B}$, then the finite simple algebras of $\mathscr{V}$ all belong to $H S(\mathbf{B})$.

In practice it may seem hard to verify whether a given variety has FHAP. In $\S 5$ two criteria are presented. The first one is of a global nature. It says that if $\mathscr{V}$ is an arithmetical variety and all of its finite subdirectly irreducible algebras have congruence lattices which possess a unique coatom, then $\mathscr{V}$ has FHAP. The second criterion is a local one: It provides a set of conditions which, if satisfied by a class $\mathscr{K}$ of finite directly indecomposable algebras in a locally finite variety ensure that $\mathscr{K}$ generates a variety with FHAP.

In $\S 6$ we define and investigate the derived varieties of a locally finite variety with FHAP. The notions and results are illustrated by a large number of examples, mostly known from the literature.

In $\$ 7$, finally, we are able to apply the theory developed in the previous sections. We examine the relationship between the structure and cardinality of the finite free algebras in a variety with FHAP and those of the free algebras in the prime variety and the derived varieties. Some of the examples from the literature to which our methods apply are listed at the end of the paper, in Tables 1 and 2.

A locally finite variety $\mathscr{V}$ has FHAP hereditarily, if not only it itself has FHAP, but also all varieties derived from $\mathscr{V}$, and their derived varieties, and so on. In $\S 8$ we make this notion precise, and show that any such variety must be arithmetical. We 
do not know of a nice characterization of these varieties, but are able to show that all congruence relative Stone varieties (Blok, Köhler and Pigozzi [1984]) have FHAP hereditarily.

We conclude this introduction with four typical examples described in the literature. As we shall see, all of them have FHAP, and have arithmetical and semisimple prime varieties. The structure theorems we quote in these examples will all follow from the results of the paper. The examples will serve as a point of reference at various stages of our exposition.

EXAMPLE 1.1. Let $\mathscr{S}$ denote the variety of Stone algebras. An algebra $\mathbf{A}=$ $\left\langle A, \wedge, \vee, 0,1,{ }^{*}\right\rangle$ is in $\mathscr{S}$ if and only if $\langle A, \wedge, \vee, 0,1\rangle$ is a bounded distributive lattice and ${ }^{*}$ is a pseudo-complement operator satisfying the equation $x^{*} \vee x^{* *}=1$.

(a) (Grätzer and Schmidt [1957]). A finite distributive lattice $\mathbf{L}$ is a reduct of a member of $\mathscr{S}$ if and only if $\mathbf{L}$ is a direct product of finite distributive lattices with exactly one atom.

(b) (Balbes and Horn [1970]). The lattice reduct of $\mathbf{F}_{\mathscr{S}}(n)$, the free Stone algebra on $n$ free generators, is isomorphic to $\prod_{i=0}^{n}\left(\mathbf{1} \oplus \mathbf{F}_{\mathscr{D}}(i) \oplus \mathbf{1}\right)^{\left({ }_{i}^{n}\right)}$ where $\mathbf{F}_{\mathscr{D}}(i)$ denotes the free distributive lattice on $i$ free generators.

EXAMPLE 1.2. A Brouwerian algebra is an algebra $\langle A, \wedge, \vee, \rightarrow, 1\rangle$ in which $\langle A, \wedge, \vee, 1\rangle$ is a distributive lattice with unit and $\rightarrow$ is relative pseudo-complementation. Let $\mathscr{B}_{k}$ denote the variety of Brouwerian algebras generated by a $k$-element chain.

(a) (Köhler [1973], Horn [1969]). For every finite $k \geqslant 2$, each finite directly indecomposable algebra in $\mathscr{B}_{k}$ is order isomorphic to a lattice $\mathbf{1} \oplus \mathbf{L}$ where $\mathbf{L}$ is in $\mathscr{B}_{k-1}$.

(b) (Köhler [1973]). For $k \geqslant 2, \mathbf{F}_{\mathscr{B}_{k}}(n) \cong \prod_{i=0}^{n-1}\left(\mathbf{1} \oplus \mathbf{F}_{\mathscr{B}_{k-1}}(i)\right)^{\left(\begin{array}{c}n \\ i\end{array}\right)}$.

EXAMPLE 1.3. Let $A$ be any nonempty finite set and let $\alpha$ be any equivalence relation on $A$. Suppose $\alpha$ has $k$ equivalence classes and the cardinalities of these classes are $c_{1}, c_{2}, \ldots, c_{k}$. Let $\mathscr{E}$ denote the variety generated by the algebra $\mathbf{A}=\langle A, F\rangle$, where $F$ consists of all operations on $A$ that preserve $\alpha$.

(a) (McKenzie [1982, p. 83]). The set of cardinalities of finite algebras in $\mathscr{E}$, that is, the spectrum of $\mathscr{E}$, is the multiplicative closure of $\{1\} \cup\left\{c_{1}^{m}+\cdots+c_{k}^{m} \mid m \geqslant 0\right\}$.

(b) (Demetrovics, Hannak and Ronyai $[\mathbf{1 9 8 2}]) . \mathbf{F}_{\mathscr{E}}(n)$ has cardinality

$$
\prod_{n_{1}+\cdots+n_{k}=n}\left(c_{1}^{m}+c_{2}^{m}+\cdots+c_{k}^{m}\right)^{\left(n_{1}, n_{2}, \cdots, n_{k}\right)}, \quad m=c_{1}^{n_{1}} c_{2}^{n_{2}} \cdots c_{k}^{n_{k}}
$$

EXAMPle 1.4 (BLoK [1976]). An interior algebra is an algebra $(A, \vee, \wedge, \Rightarrow, \square, 0,1)$ where $(A, \vee, \wedge, \Rightarrow, 0,1)$ is a Boolean algebra $(\Rightarrow$ denotes relative complementation) and $\square$ is an interior operator, satisfying $\square 1=1, \square(x \wedge y)=\square x \wedge \square y, \square x \leqslant x$ and $\square x=\square \square x$. Let $\mathbf{I}_{k}$ be the interior algebra of all subsets of the set $\{1, \ldots, k-1\}$, with $\square x=\bigcup\{[1, n]:[1, n] \subseteq x, n<k\}$, for $x \subseteq\{1, \ldots, k-1\}$. We denote by $\mathscr{I}_{k}$ the variety generated by $\mathbf{I}_{k}$ and by $\mathscr{I}_{k}^{-}$the reduct of $\mathscr{I}_{k}$ obtained by deleting the constant 0 . Let $\tilde{\mathscr{I}}_{k}$ and $\tilde{\mathscr{I}}_{k}^{-}$denote the dual varieties of $\mathscr{I}_{k}$ and $\mathscr{I}_{k}^{-}$, respectively. 
Then for $2 \leqslant k<\omega$

$$
\left|F_{\mathscr{F}_{k}}(n)\right|=\left(\left|F_{\mathscr{F}_{\bar{k}-1}}(n)\right|+\left|F_{\tilde{\mathcal{F}}_{\bar{k}-1}}(n)\right|\right)^{2^{n}} .
$$

2. Definitions and notation. For an algebra $\mathbf{A}=\left\langle A, f_{j}\right\rangle_{j \in J}$, we denote the congruence lattice of $\mathbf{A}$ by $\operatorname{Co}(\mathbf{A})$. The largest congruence on $\mathbf{A}$ is written $\nabla_{A}$ and the smallest is $\Delta_{A}$. So $\nabla_{A}=A \times A$ and $\Delta_{A}=\{(a, a) \mid a \in A\}$. Usually we write $\nabla$ and $\Delta$ when $A$ is clear from the context. For $a \in A$ and $\theta$ an equivalence relation on $A$, $a / \theta$ denotes the equivalence class of $a$.

Let $\mathscr{K}$ be a class of algebras. By $\mathscr{K}_{F}, \mathscr{K}_{S}, \mathscr{K}_{S I}$ we denote the finite algebras in $\mathscr{K}$, the simple algebras in $\mathscr{K}$, and the subdirectly irreducible algebras in $\mathscr{K}$, respectively. We adopt the convention that simple algebras and subdirectly irreducible algebras are nontrivial. An algebra $\mathbf{A}$ is directly indecomposable if $\mathbf{A}$ is nontrivial and is not isomorphic to a direct product of two nontrivial algebras. Let $\mathscr{K}_{D I}$ denote all directly indecomposable algebras in $\mathscr{K}$. We let $\mathscr{K}_{F S}=\mathscr{K}_{F} \cap \mathscr{K}_{S}, \mathscr{K}_{F S I}=$ $\mathscr{K}_{F} \cap \mathscr{K}_{S I}$, and $\mathscr{K}_{F D I}=\mathscr{K}_{F} \cap \mathscr{K}_{D I}$.

For a class $\mathscr{K}$ of algebras of the same similarity type, the four operators $I(\mathscr{K})$, $H(\mathscr{K}), S(\mathscr{K})$ and $P(\mathscr{K})$ have their usual meanings. We also have $\mathbf{A} \in P_{S D}(\mathscr{K})$ if $\mathbf{A}$ is a subdirect product of algebras in $\mathscr{K}$ and $\mathbf{A} \in P_{F}(\mathscr{K})$ if there is an $n$, $1 \leqslant n<\omega$, with $\mathbf{A} \cong \mathbf{A}_{1} \times \cdots \times \mathbf{A}_{n}, \mathbf{A}_{i} \in \mathscr{K} ; i=1, \ldots, n$.

If $\mathscr{V}$ is a variety, then the free algebra in $\mathscr{V}$ on $n$ free generators is denoted $\mathbf{F}_{\mathscr{V}}(n)$. We let $\mathscr{V}_{0}=H S P\left(\mathscr{V}_{F S}\right) ; \mathscr{V}_{0}$ is the prime variety associated with $\mathscr{V}$.

A congruence $\beta$ on $\mathbf{A}$ is a factor congruence if there exists $\beta^{\prime} \in \operatorname{Co}(\mathbf{A})$ such that $\beta \cap \beta^{\prime}=\Delta_{A}$ and $\beta \circ \beta^{\prime}=\nabla_{A}$. The pair $\left\{\beta, \beta^{\prime}\right\}$ forms a factorizing pair for $\mathbf{A}$. The factorizing pair $\left\{\beta, \beta^{\prime}\right\}$ is nontrivial if $\left\{\beta, \beta^{\prime}\right\} \neq\{\nabla, \Delta\}$. If $\left\{\beta, \beta^{\prime}\right\}$ is a factorizing pair for $\mathbf{A}$, then $\mathbf{A} \cong A / \beta \times A / \beta^{\prime}$. A nontrivial algebra $\mathbf{A}$ is directly indecomposable if and only if the only factorizing pair for $\mathbf{A}$ is $\left\{\nabla_{A}, \Delta_{A}\right\}$. Note that if $\operatorname{Co}(\mathbf{A})$ is distributive and if $\left\{\beta_{1}, \beta_{2}\right\}$ and $\left\{\beta_{1}, \beta_{3}\right)$ are factorizing pairs for $\mathbf{A}$, then $\beta_{2}=\beta_{3}$ since complements are unique in a distributive lattice. For the general case we have the following easily proved result.

LEMMA 2.1. Let $\left\{\beta_{1}, \beta_{2}\right\}$ and $\left\{\beta_{1}, \beta_{3}\right\}$ each be factorizing pairs for $\mathbf{A}$ and suppose $\beta_{2} \geqslant \beta_{3}$. Then $\beta_{2}=\beta_{3}$.

We say $\left\{\beta_{1}, \ldots, \beta_{n}\right\} \subseteq \operatorname{Co}(\mathbf{A})$ is a factorizing set if $\beta_{1} \wedge \cdots \wedge \beta_{n}=\Delta_{A}$ and for each $i, \beta_{i} \circ\left(\bigwedge_{j \neq i} \beta_{j}\right)=\nabla_{A}$. It is known that if $\left\{\beta_{1}, \ldots, \beta_{n}\right\}$ is a factorizing set for $\mathbf{A}$, then $\mathbf{A} \cong \mathbf{A} / \beta_{1} \times \cdots \times \mathbf{A} / \beta_{n}$. The factorizing set $\left\{\beta_{1}, \ldots, \beta_{n}\right\}$ is called a complete factorizing set if each $\mathbf{A} / \beta_{i}$ is directly indecomposable. Note that every nontrivial finite algebra has a complete factorizing set. In particular, if $\mathbf{A}$ is directly indecomposable, then $\{\Delta\}$ is a complete factorizing set.

An algebra $A$ satisfies the Fraser-Horn Property (FHP) if for all $\theta \in \operatorname{Co}(\mathbf{A})$ and all factorizing pairs $\left\{\beta_{1}, \beta_{2}\right\}$ for $\mathbf{A}, \theta=\left(\theta \vee \beta_{1}\right) \wedge\left(\theta \vee \beta_{2}\right)$. A variety $\mathscr{V}$ has FHP if $\mathbf{A}$ has FHP for all $A \in \mathscr{V}$. This property was introduced in Fraser and Horn [1970] and in the unpublished manuscript by T. K. Hu [1970]. It is known that $\mathscr{V}$ has FHP if and only if there are no skew congruences on finite products, i.e., for all $\mathbf{A}_{1}, \mathbf{A}_{2} \in \mathscr{V}$, every $\theta \in \operatorname{Co}\left(\mathbf{A}_{1} \times \mathbf{A}_{2}\right)$ is a product congruence $\theta_{1} \times \theta_{2}, \theta_{i} \in \operatorname{Co}\left(\mathbf{A}_{i}\right)$, 
i.e., $\left(x_{1}, x_{2}\right) \equiv\left(y_{1}, y_{2}\right)(\theta)$ if and only if $x_{1} \equiv y_{1}\left(\theta_{1}\right)$ and $x_{2} \equiv y_{2}\left(\theta_{2}\right)$. Also, if $\mathscr{V}$ has FHP, then for all $\mathbf{A}_{1}, \mathbf{A}_{2} \in \mathscr{V}, \operatorname{Co}\left(\mathbf{A}_{1} \times \mathbf{A}_{2}\right) \cong \operatorname{Co}\left(\mathbf{A}_{1}\right) \times \operatorname{Co}\left(\mathbf{A}_{2}\right)$. If $\mathscr{V}$ is congruence distributive, then $\mathscr{V}$ has FHP. Fraser and Horn [1970] give a Mal'cev condition equivalent to FHP and they derive as a special case:

LEMMA 2.2. $\mathscr{V}$ has FHP if there are binary terms + and $\cdot$ and elements 0 , $1 \in \mathbf{F}_{\mathscr{V}}(3)$ such that for all $x \in F_{\mathscr{V}}(3), x \cdot 1=x+0=0+x=x$ and $x \cdot 0=0$.

For example, the variety of rings with unit has FHP.

A finite algebra $\mathbf{A}$ has the Apple Property (AP) if for all $\beta \in \operatorname{Co}(\mathbf{A})$ if $\beta<\nabla$ is a factor congruence with $\mathbf{A} / \beta$ directly indecomposable, then the interval lattice $[\beta, \nabla]$ has exactly one coatom. A variety $\mathscr{V}$ has AP if $A \in \mathscr{V}_{F D I}$ implies $\operatorname{Co}(\mathbf{A})$ has exactly one coatom or, equivalently, if all $\mathrm{A} \in \mathscr{V}_{F}$ have AP. Typically in this paper, if $\mathscr{V}$ has $\mathrm{AP}$ and $\mathbf{A} \in \mathscr{V}_{F D I}$, then $\alpha$ will denote the coatom of $\operatorname{Co}(\mathbf{A})$. If an algebra (a variety) with FHP also has the Apply Property, we say it has FHAP. We will see later that the varieties in Examples 1.1-1.4 all have FHAP. The variety of distributive lattices has FHP, but does not have the Apple Property: for example, the 3-element chain does not have the Apple Property. The variety of Boolean groups has the Apple Property, since the only directly indecomposable Boolean group is the 2-element group $\mathbf{Z}_{2}$, but fails to have FHP: $\operatorname{Co}\left(\mathbf{Z}_{2}^{2}\right) \cong \mathbf{M}_{3}$.

We note that algebras whose congruence lattices are of the form $\mathbf{L} \oplus \mathbf{1}$ for $\mathbf{L}$ a bounded lattice arise naturally in work on the representation of algebraic lattices as congruence lattices and in the study of local rings. However, the only paper we are aware of in which the Apple Property appears as a general varietal condition is McKenzie [1984].

General texts relevant to the material covered in this paper include Balbes and Dwinger [1974], Burris and Sankappanavar [1981], and Grätzer [1979].

3. Congruence conditions associated with FHAP. In this section we examine some of the consequences of the assumption that a variety $\mathscr{V}$ has FHAP for the lattices of congruences of the (finite) algebras in $\mathscr{V}$.

First we show that FHAP yields unique factorization, the number of factors being equal to the number of coatoms of the congruence lattice.

Lemma 3.1. Let $\mathbf{A}$ be a finite algebra with FHAP. Suppose $\left\{\alpha_{1}, \ldots, \alpha_{n}\right\}$ is the set of coatoms of $\operatorname{Co}(\mathbf{A})$ and suppose $\left\{\beta_{1}, \ldots, \beta_{m}\right\}$ is a complete factorizing set for $\mathbf{A}$. Then $n=m$ and after suitable relabeling, $\beta_{i} \leqslant \alpha_{i}, i=1, \ldots, n$, and $\beta_{j} \nless \alpha_{i}$ for $i \neq j$.

Proof. For $\theta \in \operatorname{Co}(\mathbf{A}), \operatorname{Coat}(\theta)$ denotes $\{\alpha \mid \theta \leqslant \alpha$ and $\alpha$ is a coatom of $\operatorname{Co}(\mathbf{A})\}$. If $n=1$, then $\mathbf{A}$ is directly indecomposable and hence $m=1$ and $\beta_{1}=\Delta$. So assume $n \geqslant 2$. For $i \neq j$ we have $\operatorname{Coat}\left(\beta_{i}\right) \cap \operatorname{Coat}\left(\beta_{j}\right)=\varnothing$ and $\operatorname{Coat}\left(\beta_{i}\right) \neq \varnothing$. Moreover by AP, $\left|\operatorname{Coat}\left(\beta_{i}\right)\right|=1$. Let $\alpha$ be any coatom of $\operatorname{Co}(\mathbf{A})$ and suppose $\alpha \neq \beta_{j}$ for all $j=1, \ldots, m$. By FHP, for all $j$,

$$
\alpha=\left(\alpha \vee \beta_{j}\right) \wedge\left(\alpha \vee \bigwedge_{i \neq j} \beta_{i}\right)=\nabla \wedge\left(\alpha \vee \bigwedge_{i \neq j} \beta_{i}\right)=\alpha \vee \bigwedge_{i \neq j} \beta_{i}
$$


So $\alpha \geqslant \bigwedge_{i \neq j} \beta_{i}$ for all $j$ but $\nabla=\bigvee_{1 \leqslant j \leqslant m}\left(\bigwedge_{i \neq j} \beta_{i}\right)$, a contradiction. So for every $\alpha_{i}$, $1 \leqslant i \leqslant n$, there exists a unique $\beta_{k}$ with $\alpha_{i} \geqslant \beta_{k}$ and $\alpha_{i} \neq \beta_{j}$ for $j \neq k$. So $m=n$ and relabeling as necessary completes the proof.

A bounded lattice $\mathbf{L}$ is dually pseudo-complemented if for all $x \in L$ there exists an $x^{+} \in L$ with $x \vee x^{+}=1$ and if $x \vee y=1$, then $x^{+} \leqslant y$. If $\mathbf{L}$ is dually pseudo-complemented then $x^{++} \leqslant x$. The skeleton of $\mathbf{L}$ is $\left\{x^{+} \mid x \in L\right\}$. $\mathbf{L}$ is skeleton distributive if $y=\left(y \vee x^{+}\right) \wedge\left(y \vee x^{++}\right)$for all $x, y \in L$. Note that, in general, $\mathbf{L}$ being distributive does not imply $\mathbf{L}$ is skeleton distributive; however, if $\mathbf{L}$ satisfies $x^{+} \wedge x^{++}=0$, then it does.

LeMma 3.2. Let $\mathbf{L}$ be a finite lattice with 1 in which every coatom has a dual pseudo-complement. Then $\mathbf{L}$ is dually pseudo-complemented, and the dual pseudo-complement of an element is uniquely determined by the coatoms above it.

Proof. Let $a_{1}, \ldots, a_{n}$ be the coatoms of $\mathbf{L}$ and let $a_{1}^{+}, \ldots, a_{n}^{+}$be their dual pseudo-complements. As before, for $x \in L, \operatorname{Coat}(x)=\{a \in L \mid a \geqslant x, a$ a coatom $\}$. We claim that for $x \in L, x^{+}=\bigvee\left\{a^{+} \mid a \in \operatorname{Coat}(x)\right\}$. If $x \vee \vee\left\{a^{+} \mid a \in \operatorname{Coat}(x)\right\}<$ 1 , then $a^{+} \leqslant a$ for some $a \in \operatorname{Coat}(x)$, contradicting $a \vee a^{+}=1$. Thus

$$
x \vee \vee\left\{a^{+} \mid a \in \operatorname{Coat}(x)\right\}=1 .
$$

Now assume $x \vee y=1$. Then, for all $a \in \operatorname{Coat}(x), a \vee y \geqslant x \vee y=1$, and hence $y \geqslant a^{+}$. Thus $y \geqslant \vee\left\{a^{+} \mid a \in \operatorname{Coat}(x)\right\}$, and it follows that

$$
x^{+}=\bigvee\left\{a^{+} \mid a \in \operatorname{Coat}(x)\right\} .
$$

Lemma 3.3. Let $\mathbf{A}$ be a finite algebra with FHAP. Suppose $\alpha$ is a coatom of $\operatorname{Co(\mathbf {A})}$ and $\left\{\beta_{1}, \ldots, \beta_{n}\right\}$ is a complete factorizing set for $\mathbf{A}$. Then $\alpha$ has a dual pseudo-complement $\alpha^{+}=\Lambda_{\beta_{i} \nless \alpha} \beta_{i}$.

Proof. Let $\alpha_{1}, \ldots, \alpha_{n}$ be the coatoms of $\operatorname{Co}(\mathbf{A})$. By Lemma 3.1 the complete factorizing set is $\left\{\beta_{1}, \ldots, \beta_{n}\right\}$ with $\alpha_{i} \geqslant \beta_{i}, i=1, \ldots, n$, and $\alpha_{i} \neq \beta_{j}, i \neq j$. Note $\alpha_{i} \vee \Lambda_{j \neq i} \beta_{j} \geqslant \beta_{i} \vee \Lambda_{j \neq i} \beta_{j}=\nabla$. We claim $\alpha_{i}^{+}=\bigwedge_{j \neq i} \beta_{j}$. Suppose $\alpha_{i} \vee \delta=\nabla$. Then $\beta_{i} \vee \delta=\nabla$ as well, for otherwise we would have $\delta \leqslant \beta_{i} \vee \delta \leqslant \alpha_{j}$, for some $j$, $1 \leqslant j \leqslant n$, and hence $i=j$, yielding the contradiction $\alpha_{i}=\alpha_{i} \vee \delta=\nabla$. By FHP, $\delta=\left(\delta \vee \beta_{i}\right) \wedge\left(\delta \vee \bigwedge_{j \neq i} \beta_{j}\right)=\delta \vee\left(\bigwedge_{j \neq i} \beta_{j}\right)$, i.e., $\bigwedge_{j \neq i} \beta_{j} \leqslant \delta$.

THEOREM 3.4. Let $\mathbf{A}$ be a finite algebra with FHAP and suppose $\operatorname{Co}(\mathbf{A})$ has coatoms $\alpha_{1}, \ldots, \alpha_{n}$. Then

(i) There exists a complete factorizing set $\left\{\beta_{1}, \ldots, \beta_{n}\right\}$ with $\alpha_{i} \geqslant \beta_{j}$ if and only if $i=j$.

(ii) $\mathrm{Co}(\mathbf{A})$ is dually pseudo-complemented.

(iii) $\mathbf{A} / \alpha_{i}^{++}$is directly indecomposable, Coat $\left(\alpha_{i}^{++}\right)=\left\{\alpha_{i}\right\}$, and $\beta_{i}=\alpha_{i}^{++}$. Hence $\left\{\alpha_{1}^{++}, \ldots, \alpha_{n}^{++}\right\}$is the unique complete factorizing set for $\mathbf{A}$.

(iv) If $\gamma \in \operatorname{Co}(\mathbf{A})$ and $\operatorname{Coat}(\gamma)=\left\{\alpha_{i}\right\}$, then $\alpha_{i}^{++} \leqslant \gamma<\nabla$.

(v) For all $\gamma, \delta \in \operatorname{Co}(\mathbf{A}), \gamma^{+} \wedge \gamma^{++}=\Delta, \gamma^{+} \circ \gamma^{++}=\nabla$, and $\delta=\left(\delta \vee \gamma^{+}\right) \wedge$ $\left(\delta \vee \gamma^{++}\right)$, i.e., $\operatorname{Co}(\mathbf{A})$ is skeleton distributive. 
Proof. (i) is a restatement of Lemma 3.1 and (ii) follows from Lemmas 3.3 and 3.2. To prove (v) let $\gamma \in \operatorname{Co}(\mathbf{A})$ and suppose $\operatorname{Coat}(\gamma)=\left\{\alpha_{i} \mid i \in J\right\}$. Then $\operatorname{Coat}\left(\gamma^{+}\right)$ $=\left\{\alpha_{i} \mid i \notin J\right\}$. Moreover, since $\left\{\bigwedge_{i \in J} \beta_{i}, \bigwedge_{i \notin J} \beta_{i}\right\}$ form a factorizing pair it is easily seen that $\operatorname{Coat}\left(\gamma^{+}\right)=\operatorname{Coat}\left(\bigwedge_{i \notin J} \beta_{i}\right)$ and hence $\Lambda_{i \notin J} \beta_{i} \geqslant \gamma^{+}$. By FHP

$$
\gamma^{+}=\left(\gamma^{+} \vee \bigwedge_{i \in J} \beta_{i}\right) \wedge\left(\gamma^{+} \vee \bigwedge_{i \notin J} \beta_{i}\right)=\nabla_{A} \wedge\left(\gamma^{+} \vee \bigwedge_{i \notin J} \beta_{i}\right)=\gamma^{+} \vee \bigwedge_{i \notin J} \beta_{i}
$$

So $\gamma^{+} \geqslant \bigwedge_{i \notin J} \beta_{i}$. Thus $\gamma^{+}=\bigwedge_{i \notin J} \beta_{i}$. Similarly $\gamma^{++}=\bigwedge_{i \in J} \beta_{i}$. So $\left\{\gamma^{+}, \gamma^{++}\right\}$is a factorizing pair and this with FHP gives (v). Specializing the formula $\gamma^{++}=\Lambda_{i \in J} \beta_{i}$ to the case that $\gamma$ is a coatom $\alpha_{i}$ gives (iii). For (iv), if $\operatorname{Coat}(\gamma)=\left\{\alpha_{i}\right\}$, then by Lemma $3.2 \gamma^{+}=\alpha_{i}^{+}$and since $\gamma^{++} \leqslant \gamma$ always holds, the proof is complete.

We have the following sharp converse.

THEOREM 3.5. Let A be any finite algebra. Suppose $\operatorname{Co}(\mathbf{A})$ is a dually pseudocomplemented lattice that is skeleton distributive and satisfies $\gamma^{+} \circ \gamma^{++}=\nabla$ for all $\gamma \in \operatorname{Co}(\mathbf{A})$. Then A has FHAP.

Proof. Let $\{\beta, \gamma\}$ be any factorizing pair for A. $\beta \vee \gamma=\nabla$ implies $\beta \geqslant \gamma^{+}$. By hypothesis $\gamma^{+} \circ \gamma^{++}=\nabla$ and hence $\beta \circ \gamma^{++}=\nabla$. Since $\gamma \geqslant \gamma^{++}, \beta \wedge \gamma^{++}=\Delta$. So $\left\{\beta, \gamma^{++}\right\}$is also a factorizing pair, and by Lemma $2.1 \gamma=\gamma^{++}$. Similarly $\gamma^{+}=\beta$. So every factorizing pair is of the form $\left\{\gamma^{+}, \gamma^{++}\right\}$and $\operatorname{Co}(\mathbf{A})$ skeleton distributive implies $A$ has FHP. Let $\beta$ be any factorizing congruence for $\mathbf{A}$ with $\mathbf{A} / \beta$ directly indecomposable. Let $\alpha$ be any coatom in $\operatorname{Co}(\mathbf{A})$ with $\beta \leqslant \alpha$. Then $\beta=\left(\beta \vee \alpha^{+}\right) \wedge$ $\left(\beta \vee \alpha^{++}\right)$. Since $\alpha^{+} \circ \alpha^{++}=\nabla$ it follows that $\nabla=\left(\beta \vee \alpha^{+}\right) \circ\left(\beta \vee \alpha^{++}\right)$. That $\mathbf{A} / \beta$ is directly indecomposable implies $\beta=\beta \vee \alpha^{++}$and hence $\beta \geqslant \alpha^{++}$. The only coatom in the interval $\left[\alpha^{++}, \nabla\right]$ is $\alpha$. Thus $\mathbf{A}$ has the Apple Property.

We will be particularly interested in varieties $\mathscr{V}$ such that the prime variety $\mathscr{V}_{0}$ is arithmetical. As it turns out, just this assumption implies that for $\mathbf{A} \in \mathscr{V}_{F}, \operatorname{Co}(\mathbf{A})$ is dually pseudo-complemented. This result was pointed out to us by Ralph McKenzie and a more general result is proved in McKenzie and Hobby [1986] using tame congruence theory. We give an alternative proof.

THEOREM 3.6. Let $\mathbf{A}$ be any finite algebra and let $\alpha$ be any congruence relation such that $\mathbf{A} / \alpha$ is simple and $\mathbf{A} / \alpha$ generates an arithmetical variety. Then $\alpha$ has a dual pseudo-complement in $\mathrm{Co}(\mathbf{A})$.

Proof. Suppose $\delta_{1}, \delta_{2} \in \operatorname{Co}(\mathbf{A})$ are such that $\delta_{1} \vee \alpha=\delta_{2} \vee \alpha=\nabla$. It suffices to show $\left(\delta_{1} \wedge \delta_{2}\right) \vee \alpha=\nabla$. The congruence $\alpha$ is a coatom in $\operatorname{Co}(\mathbf{A})$ since $\mathbf{A} / \alpha$ is simple. So it suffices to show $\delta_{1} \wedge \delta_{2} \nless \alpha$. Assume not, and if necessary, take a homomorphic image, to give $\delta_{1} \wedge \delta_{2}=\Delta$. That $\delta_{1} \vee \alpha=\delta_{2} \vee \alpha=\nabla$ and that $\mathrm{A} / \alpha$ is simple and generates a congruence permutable variety guarantee, using Lakser [1982], that there exist two congruence classes $A_{1}$ and $A_{2}$ of $\alpha$ and elements $b_{1}$, $b_{2} \in A_{1}$ and $c_{1}, c_{2} \in A_{2}$ with $b_{i} \equiv c_{i}\left(\delta_{i}\right), i=1,2$. By Werner [1970], $\mathbf{A} / \alpha$ is functionally complete, so there is a polynomial function $t: A^{3} \rightarrow A$ given by $t(x, y, z)=p\left(x, y, z, a_{1}, \ldots, a_{n}\right)$, with $a_{1}, \ldots, a_{n}$ fixed elements of $A$, such that $t$ is a ternary discriminator function on $\mathbf{A} / \alpha$, that is, $t(x, y, z) \equiv z(\alpha)$ if $x \equiv y(\alpha)$ and 


$$
\begin{aligned}
t(x, y, z) \equiv x(\alpha) \text { if } x & \neq \equiv(\alpha) \text {. Let } \\
a & =t\left(t\left(b_{1}, c_{2}, c_{2}\right), t\left(b_{1}, c_{2}, c_{2}\right), t\left(b_{1}, c_{2}, c_{2}\right)\right), \\
b & =t\left(t\left(c_{1}, c_{2}, c_{2}\right), t\left(b_{1}, c_{2}, c_{2}\right), t\left(b_{1}, c_{2}, c_{2}\right)\right), \\
c & =t\left(t\left(c_{1}, c_{2}, c_{2}\right), t\left(b_{1}, c_{2}, c_{2}\right), t\left(b_{1}, b_{2}, c_{2}\right)\right), \\
d & =t\left(t\left(b_{1}, c_{2}, c_{2}\right), t\left(b_{1}, c_{2}, c_{2}\right), t\left(b_{1}, b_{2}, c_{2}\right)\right) .
\end{aligned}
$$

It is easily verified that $a \in A_{1}, b, c, d \in A_{2}, a \equiv b\left(\delta_{1}\right), b \equiv c\left(\delta_{2}\right), c \equiv d\left(\delta_{1}\right)$, and $d \equiv a\left(\delta_{2}\right)$. The elements $a, b, c, d$ are all distinct for otherwise $\delta_{1} \wedge \delta_{2}=\Delta$ would be violated. See Figure 1 . For $x, y \in A$, let $g_{x y}(z)=t(x, y, z)$ be a unary operation on $A$. Let $n \geqslant 2$ be arbitrary and for $1 \leqslant k \leqslant n$ let $e_{k}=g_{c c}^{n-k}\left(g_{b c}^{k}(c)\right)$.

Claim: All the $e_{k}, 1 \leqslant k \leqslant n$, are distinct. For suppose $e_{i}=e_{j}$ for $i<j$. Define $a^{\prime}=g_{c c}^{n-j}\left(g_{a c}^{j-i}\left(g_{b c}^{i}(c)\right)\right)$ and $d^{\prime}=g_{c c}^{n-j}\left(g_{d c}^{j-i}\left(g_{b c}^{i}(c)\right)\right)$. Then $a^{\prime} \in A_{1}$ and $d^{\prime} \in A_{2}$ since for all $z \in A, g_{a c}(z) \equiv a(\alpha)$ and $g_{c c}(z) \equiv g_{d c}(z) \equiv g_{b c}(z) \equiv z(\alpha)$. Also $a^{\prime} \equiv$ $e_{j}\left(\delta_{1}\right), d^{\prime} \equiv e_{i}\left(\delta_{1}\right)$, and $a^{\prime} \equiv d^{\prime}\left(\delta_{2}\right)$. Hence if $e_{i}=e_{j}$, then $a^{\prime} \equiv d^{\prime}\left(\delta_{1} \wedge \delta_{2}\right)$ which is impossible. So the $e_{k}, 1 \leqslant k \leqslant n$, are all distinct and since $A$ is finite and $n$ is arbitrary, the proof is complete.

COROllaRy 3.7. Let $\mathbf{A}$ be any finite algebra and suppose every simple homomorphic image of $\mathbf{A}$ generates an arithmetical variety. Then $\mathrm{Co}(\mathbf{A})$ is a dually pseudo-complemented lattice.

Proof. Apply Theorem 3.6 and Lemma 3.2.

The existence of a polynomial that behaves as a ternary discriminator for simple algebras in arithmetic varieties has been estblished in Burris [1982] and Pixley [1985].

We conclude this section with two results which will be useful later.

Lemma 3.8. Let $\mathscr{V}$ be a variety with FHP. Suppose $\mathbf{A} \in \mathscr{V}_{F}$ with $\left\{\beta_{1}, \ldots, \beta_{m}\right\}$ a complete factorizing set for $\mathbf{A}$ and suppose each interval $\left[\beta_{i}, \nabla\right]$ in $\operatorname{Co}(\mathbf{A})$ has exactly one coatom, $i=1, \ldots, m$. Then every directly indecomposable homomorphic image of A has a congruence lattice with exactly one coatom.

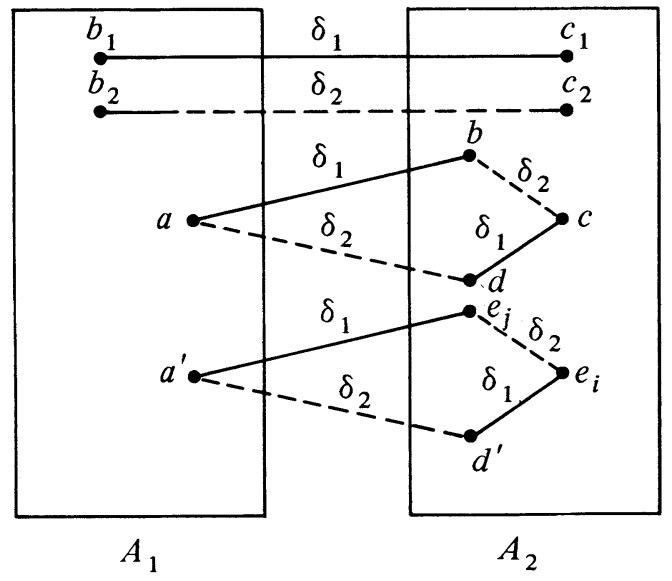


Proof. Let $\theta \in \operatorname{Co}(\mathbf{A})$ with $\mathbf{A} / \theta$ directly indecomposable. By FHP, $\theta=\left(\theta \vee \beta_{1}\right)$ $\wedge \cdots \wedge\left(\theta \vee \beta_{m}\right)$. For each $i,\left(\theta \vee \beta_{i}\right) \circ \Lambda_{j \neq i}\left(\theta \vee \beta_{j}\right)=\nabla$. Since $\mathbf{A} / \theta$ is directly indecomposable, $\theta \vee \beta_{i}=\theta$ or $\theta \vee \beta_{i}=\nabla, i=1, \ldots, m$, and hence for at least one $i, \theta \vee \beta_{i}=\theta$. So $\theta \geqslant \beta_{i}$ and the interval $[\theta, \nabla]$ has exactly one coatom.

COROllary 3.9. Let $\mathscr{V}$ be a locally finite variety that has FHP. Suppose for every $n<\omega, \mathbf{F}_{\mathscr{V}}(n)$ has a complete factorizing set $\left\{\beta_{1}, \ldots, \beta_{m}\right\}$ with $\left[\beta_{i}, \nabla\right]$ having exactly one coatom, $i=1, \ldots, m$. Then $\mathscr{V}$ has the Apple Property.

Proof. It suffices to show that every finite directly indecomposable algebra $\mathbf{A}$ in $\mathscr{V}$ has $\operatorname{Co}(\mathbf{A})$ with exactly one coatom. Let $\mathbf{A}$ be a homomorphic image of $\mathbf{F}_{\mathscr{V}}(n)$ for some $n$. Let $\left\{\beta_{1}, \ldots, \beta_{m}\right\}$ be a factorizing set of congruences for $\mathbf{F}_{\mathscr{V}}(n)$ with each $\left[\beta_{i}, \nabla\right]$ having exactly one coatom. Now apply Lemma 3.8.

4. The simple algebras in a variety with FHAP. Given a locally finite variety $\mathscr{V}$ with FHAP, let $\mathscr{V}_{0}$ denote the subvariety of $\mathscr{V}$ generated by all finite simple algebras in $\mathscr{V}$, i.e., $\mathscr{V}_{0}=H S P\left(\mathscr{V}_{F S}\right)$. We call $\mathscr{V}_{0}$ the prime variety of $\mathscr{V}$. In general, not much can be said about $\mathscr{V}_{0}$. We will show, however, that a mild extra assumption forces $\mathscr{V}_{0}$ to be arithmetical and semisimple. In that case the finite algebras of $\mathscr{V}_{0}$ are just products of simples.

Lemma 4.1. Let $\mathbf{A}$ be a finite algebra with FHAP. Suppose $\left\{\alpha_{1}, \ldots, \alpha_{n}\right\}$ is the set of coatoms of $\operatorname{Co}(\mathbf{A})$ and suppose $\delta=\Lambda_{1 \leqslant i \leqslant n} \alpha_{i}$. Then $\mathbf{A} / \delta \cong \mathbf{A} / \alpha_{1} \times \cdots \times \mathbf{A} / \alpha_{n}$.

Proof. Let $\left\{\beta_{1}, \ldots, \beta_{m}\right\}$ be a complete factorizing set for A. By Lemma 3.1 we may assume $m=n, \beta_{i} \leqslant \alpha_{i}$ for $i=1, \ldots, n$, and $\beta_{j} \nless \alpha_{i}$ for $i \neq j$. For each $i$, $\bigwedge_{j \neq i} \alpha_{j} \geqslant \bigwedge_{j \neq i} \beta_{j}$, so $\nabla_{A}=\beta_{i} \circ \bigwedge_{j \neq i} \beta_{j} \leqslant \alpha_{i} \circ \bigwedge_{j \neq i} \alpha_{j}$. So

$$
\mathbf{A} / \delta \cong(\mathbf{A} / \delta) /\left(\alpha_{1} / \delta\right) \times \cdots \times(\mathbf{A} / \delta) /\left(\alpha_{n} / \delta\right) \cong \mathbf{A} / \alpha_{1} \times \cdots \times \mathbf{A} / \alpha_{n} .
$$

Definition 4.2. A class $\mathscr{K} \subset \mathscr{V}_{S}$ is said to be multiplicative if whenever $\mathbf{B} \in \mathscr{V}_{F}$ is an irredundant subdirect product of $\mathbf{B}_{1}, \ldots, \mathbf{B}_{m} \in \mathscr{K}$, then $\mathbf{B}=\prod_{1 \leqslant i \leqslant m} \mathbf{B}_{i}$. (A is an irredundant subdirect product of $\mathbf{B}_{1}, \ldots, \mathbf{B}_{m}$ if $\mathbf{A}$ is subdirectly embedded in $\prod_{1 \leqslant i \leqslant m} \mathbf{B}_{i}$ and for all $i$ no projection of $\mathbf{A}$ onto $\prod_{j \neq i} \mathbf{B}_{j}$ is one-to-one.)

Thus if $\mathscr{V}$ has FHAP then by Lemma $4.1 \mathscr{V}_{F S}$ is a multiplicative class.

THEOREM 4.3. Let $\mathscr{V}$ be a locally finite variety with FHAP. Suppose $\mathscr{V}$ also has $S\left(\mathscr{V}_{F S}\right) \subseteq P\left(\mathscr{V}_{S}\right)$. Then $\mathscr{V}_{0}$ is arithmetical, $\left(\mathscr{V}_{0}\right)_{F} \subseteq P\left(\mathscr{V}_{S}\right)$, and every finitely generated subvariety of $\mathscr{V}_{0}$ is directly representable.

Proof. For any $n<\omega$ let $\mathbf{F}$ denote $\mathbf{F}_{\mathscr{V}_{0}}(n)$. The algebra $\mathbf{F}$ is in $P_{S D} S\left(\mathscr{V}_{F S}\right)=$ $P_{S D}\left(P\left(\mathscr{V}_{S}\right)\right)=P_{S D}\left(\mathscr{V}_{S}\right)$. FHAP implies that $\mathscr{V}_{F S}$ is multiplicative so $\mathbf{F} \in P\left(\mathscr{V}_{S}\right)$. By FHP every homomorphic image of $\mathbf{F}$ is also in $P\left(\mathscr{V}_{S}\right)$, so $\left(\mathscr{V}_{0}\right)_{F} \subseteq P\left(\mathscr{V}_{S}\right)$. Hence every congruence lattice of a finite algebra in $\mathscr{V}_{0}$ is a Boolean lattice and it follows that $\mathscr{V}_{0}$ is congruence distributive. Let $\mathbf{B}$ be a finite algebra in $\mathscr{V}_{0}$ and let $\mathscr{W}$ be the subvariety of $\mathscr{V}_{0}$ generated by $\mathbf{B}$. Every $\mathbf{A} \in \mathscr{W}_{F}$ is a direct product of simple algebras and by Jónsson's Theorem these simple algebras are homomorphic images of subalgebras of $\mathbf{B}$. So $\mathscr{W}$ is directly representable. By McKenzie [1982], $\mathscr{W}$ is 
congruence permutable. If we let $\mathscr{W}=\operatorname{HSP}\left(\mathbf{F}_{\mathscr{V}_{0}}(3)\right)$, then it follows that $\mathbf{F}_{\mathscr{W}}(3)=$ $\mathbf{F}_{\mathscr{V}_{0}}(3)$ has permuting congruences, so $\mathscr{V}_{0}$ is congruence permutable.

The theorem has the following special version.

Corollary 4.4. Let $\mathscr{V}$ be a locally finite variety with FHAP. Suppose $S\left(\mathscr{V}_{F S}\right) \subseteq$ $\mathscr{V}_{S}$. Then every finitely generated subvariety of $\mathscr{V}_{0}$ is a ternary discriminator variety.

Proof. By Theorem $4.3 \mathscr{V}_{0}$ is arithmetical. Let $\mathscr{W}$ be any finitely generated subvariety of $\mathscr{V}_{0}$. Then $\mathscr{W}$ is generated by a finite number of finite simple algebras. A result of Pixley [1971] states tht any finitely generated arithmetical variety generated by hereditarily simple algebras is a discriminator variety.

Note that the variety $\mathscr{V}$ generated by the 6-element orthomodular lattice $\mathbf{M}_{6}$ is arithmetical (Burris and Sankappannavar [1981, p. 82]) and easily seen to have FHAP since $\mathscr{V}$ is congruence distributive and the only finite directly indecomposables are $\mathbf{M}_{6}$ and the 2-element orthomodular lattice. So $\mathscr{V}=\mathscr{V}_{0}$ and $S\left(\mathscr{V}_{F S}\right) \subseteq$ $P\left(\mathscr{V}_{S}\right)$ and $\left(\mathscr{V}_{0}\right)_{F S I} \subseteq \mathscr{V}_{S}$; however, $S\left(\mathscr{V}_{F S}\right) \nsubseteq \mathscr{V}_{S}$ since the 4-element Boolean algebra is a subalgebra of $\mathbf{M}_{6}$.

If $\mathscr{V}$ is finitely generated and has FHAP, then $\mathscr{V}_{0}$ is easily found. We first prove a lemma.

LEMma 4.5. Let $\mathscr{V}$ be a locally finite variety with FHAP, $\mathscr{K} \subseteq \mathscr{V}_{F D I}$, and $\mathbf{A} \in P_{S D}(\mathscr{K})$. If $\mathbf{B}$ is a direct factor of $\mathbf{A}$, then $\mathbf{B} \in P_{S D}(\mathscr{K})$.

Proof. Let $\gamma_{i} \in \operatorname{Co}(\mathbf{A}), i \in I$, be such that $\bigwedge_{i \in I} \gamma_{i}=\Delta$ and $\mathbf{A} / \gamma_{i} \in \mathscr{K}, i \in I$. Let $\left\{\beta, \beta^{\prime}\right\}$ be a factorizing pair of congruences, with $\mathbf{A} / \beta \cong \mathbf{B}$. By FHP $\gamma_{i}=\left(\gamma_{i} \vee \beta\right)$ $\wedge\left(\gamma_{i} \vee \beta^{\prime}\right)$, and since $\mathbf{A} / \gamma_{i}$ is directly indecomposable it follows that $\gamma_{i} \geqslant \beta$ or $\gamma_{i} \geqslant \beta^{\prime}$. Let $\gamma_{1}=\wedge_{i \in I}\left\{\gamma_{i}: \gamma_{i} \geqslant \beta, i \in I\right\}$. Then $\gamma_{1} \wedge \beta^{\prime} \leqslant \Lambda_{i \in I} \gamma_{i}=\Delta_{A}$ and $\gamma_{1} \circ \beta^{\prime}$ $\geqslant \beta \circ \beta^{\prime}=\nabla_{A}$. Thus $\left\{\gamma_{1}, \beta^{\prime}\right\}$ is a factorizing pair and it follows from Lemma 2.1 that $\gamma_{1}=\beta$. Thus $\mathbf{B} \cong \mathbf{A} / \beta \in P_{S D}\left\{\mathbf{A} / \gamma_{i}: \gamma_{i} \geqslant \beta\right\} \subseteq P_{S D} \mathscr{K}$.

Theorem 4.6. Let $\mathscr{V}=H S P(\mathbf{B})$, B finite, and suppose $\mathscr{V}$ has FHAP. Then $\mathscr{V}_{F S} \subseteq H S((\mathbf{B})$.

Proof. Let $\mathbf{A} \in \mathscr{V}_{F S}$ and choose $n$ large enough that $\mathbf{A} \in H\left(\mathbf{F}_{\mathscr{V}}(n)\right)$. There is a coatom $\alpha \in \operatorname{Co}\left(\mathbf{F}_{\mathscr{V}}(n)\right)$ with $\mathbf{F}_{\mathscr{V}}(n) / \alpha \cong \mathbf{A}$. By Theorem $3.4\left\{\alpha^{+}, \alpha^{++}\right\}$is a factorizing pair with $\mathbf{F}_{\mathscr{V}}(n) / \alpha^{++}$directly indecomposable and $\alpha$ the unique coatom in $\left[\alpha^{++}, \nabla\right]$. The algebra $\mathbf{F}_{\mathscr{V}}(n)$ is a subdirect product of members of $S(\mathbf{B})$ and each algebra $\mathbf{C}$ in $S(\mathbf{B})$ is a direct product of directly indecomposable members of $H S(\mathbf{B})$, i.e., the directly indecomposable factors of $\mathbf{C}$ are in $H(\mathbf{C}) \subseteq H S(\mathbf{B})$.

By Lemma 4.5, $\mathbf{F}_{\mathscr{\gamma}}(n) / \alpha^{++} \in P_{S D}\left(H S(\mathbf{B})_{D I}\right)$ as well, hence there exists a congruence $\gamma \in \operatorname{Co}\left(\mathbf{F}_{\mathscr{\gamma}}(n)\right)$ such that $\gamma \geqslant \alpha^{++}, \mathbf{F}_{\mathscr{\gamma}}(n) / \gamma \in H S(\mathbf{B})_{D I}$. Then $\alpha \geqslant \gamma$ and $\mathbf{A} \cong \mathbf{F}_{\mathscr{\gamma}}(n) / \alpha \in H\left(\mathbf{F}_{\mathscr{\gamma}}(n) / \gamma\right) \subseteq H S(\mathbf{B})$.

Each of the varieties given in Examples 1.1-1.4 are generated by a finite algebra and therefore Theorem 4.6 may be used to describe their simple algebras. This gives the 2-element Boolean algebra for Example 1.1, the 2-element generalized Boolean algebra for Example 1.2, the $k$-element primal algebra for Example 1.3, and the 2-element interior algebra for Example 1.4. These four examples are congruence 
distributive so Jónsson's Theorem (Jónsson [1967]) could be used in place of Theorem 4.5. However, in Example 6.6 we show FHAP does not imply any congruence identity, so Jónsson's Theorem or its generalizations cannot generally be applied in this way.

5. Sufficient conditions for FHAP. We will now provide two sets of conditions on a variety to ensure it has FHAP. The conditions of the first one are of a global nature, those of the second one are conditions on a generating set of algebras.

THEOREM 5.1. Let $\mathscr{V}$ be an arithmetical variety all of whose finite subdirectly irreducible algebras have the Apple Property. Then $\mathscr{V}$ has FHAP.

Proof. Let $\mathbf{A} \in \mathscr{V}$ be finite and suppose $\operatorname{Co}(\mathbf{A})$ has coatoms $\alpha_{1}, \ldots, \alpha_{n}, n>1$. We claim $\mathbf{A}$ is directly decomposable. Let $\beta_{1}, \ldots, \beta_{m}$ be the meet-irreducible congruences of $\mathbf{A}$. Since $\mathbf{A} / \beta_{i}$ is subdirectly irreducible it has the Apple Property by assumption, and therefore $\left[\beta_{i}\right)$ contains exactly one coatom. Suppose $\beta_{1}, \ldots, \beta_{l} \leqslant \alpha_{1}$, $\beta_{l+1}, \ldots, \beta_{m} \nless \alpha_{1}$. Then $\gamma_{1}=\bigwedge_{i=1}^{l} \beta_{i} \leqslant \alpha_{1}$, and

$$
\gamma_{1} \vee \alpha_{j}=\left(\bigwedge_{i=1}^{l} \beta_{i}\right) \vee \alpha_{j}=\bigwedge_{i=1}^{l}\left(\beta_{i} \vee \alpha_{j}\right)=\nabla,
$$

and hence $\gamma_{1} \nless \alpha_{j}, j=2, \ldots, n$. Similarly $\gamma_{2}=\bigwedge_{i=1+1}^{m} \beta_{i} \nless \alpha_{1}$.

It follows that $\gamma_{1} \vee \gamma_{2} \nless \alpha_{j}, j=1, \ldots, n$, and hence $\gamma_{1} \vee \gamma_{2}=\nabla$. Also $\gamma_{1} \wedge \gamma_{2}$ $=\wedge_{i=1}^{m} \beta_{i}=\Delta$, and since $\mathscr{V}$ is congruence permutable $\left\{\gamma_{1}, \gamma_{2}\right\}$ is a factorizing pair. Thus $\mathbf{A}$ is directly decomposable. Finally, $\mathscr{V}$ has FHP since $\mathscr{V}$ is congruence distributive, therefore $\mathscr{V}$ has FHAP.

The varieties in Examples 1.2-1.4 are all arithmetical, and their subdirectly irreducibles have linearly ordered congruence lattices, and thus have the Apple Property. Hence the varieties in these examples have FHAP. The theorem is not applicable to the variety of Stone algebras (Example 1.1), since it fails to be congruence permutable. Nor does it apply to the varieties of rings with 1 generated by $\mathbf{Z}_{p^{2}}, p$ prime (see Example 5.5), since those fail to be congruence distributive. In cases like these, and many others-cf. \$6-we can use the following theorem instead.

THEOREM 5.2. Let $\mathscr{V}$ be a locally finite variety whose term operations include three binary operations,$+ \cdot$, and $\oplus$. Suppose there is a family $\mathscr{K} \subseteq \mathscr{V}_{F D I}$ such that each of the following six conditions holds for $\mathscr{K}$ and all $\mathbf{C} \in \mathscr{K}$ :

(i) for all $n<\omega, \mathbf{F}_{\mathscr{V}}(n) \in P_{S D}(\mathscr{K})$;

(ii) $(H(\mathscr{K}))_{S}$ is multiplicative (cf. Definition 4.2);

(iii) $\mathrm{Co}(\mathbf{C})$ has exactly one coatom $\alpha$;

(iv) there exist $0_{C}, 1_{C} \in C$ such that for all $a \in C, 0_{C}+a=a+0_{C}=i_{C} \cdot a=$ $a \cdot 1_{C}=a$ and $0_{C} \cdot a=0_{C}$;

(v) if $x^{\prime}$ denotes the polynomial $x \oplus 0_{C}$, then for all $a \in 0_{C} / \alpha, a^{\prime}=0_{C}$ and for all $b \in 1_{C} / \alpha, b^{\prime}=1_{C}$;

(vi) if $a, b \in C$ and $a / \alpha \neq b / \alpha$, then there exists a unary polynomial $p$ for which $\{p(a / \alpha), p(b / \alpha)\}=\left\{p\left(0_{C} / \alpha\right), p\left(1_{C} / \alpha\right)\right\}$.

Then $\mathscr{V}$ has FHAP and $\mathscr{V}_{F S}=(H(\mathscr{K}))_{S}$. 
Proof. We first note that by (iv), if $\mathbf{A} \in \mathscr{K}$, then the elements $0_{A}$ and $1_{A}$ are uniquely determined and if $h: \mathbf{A} \rightarrow \mathbf{B} \in \mathscr{K}$ is an onto homomorphism, then $h\left(0_{A}\right)=0_{B}$ and $h\left(1_{A}\right)=1_{B}$. (Note that $0_{A}$ and $1_{A}$ need not represent algebraic constants of the similarity type of $\mathscr{V}$.) For the remainder of the proof $n$ is an arbitrary positive integer and $\mathbf{F}$ denotes $\mathbf{F}_{\mathscr{V}}(n)$. By (i) there are $\gamma_{1}, \ldots, \gamma_{m} \in \operatorname{Co}(\mathbf{F})$ with $\mathbf{F} / \gamma_{j}=\mathbf{C}_{j} \in \mathscr{K}$ and $\bigwedge_{j=1}^{m} \gamma_{j}=\Delta_{F}$. The mapping $g: \mathbf{F} \rightarrow \mathbf{C}_{1} \times \cdots \times \mathbf{C}_{m}$ is a subdirect embedding.

Since $0_{C_{i}}$ is absorptive for - and since $g$ is a subdirect embedding, if $0_{F}=$ $\left(\left(\left(z_{1} \cdot z_{2}\right) \cdot z_{3}\right) \cdots\right) \cdot z_{m}$ where $z_{i} \in F, g\left(z_{i}\right)(i)=0_{C_{i}}$, then $g\left(0_{F}\right)=\left\langle 0_{C_{1}}, \ldots, 0_{C_{m}}\right\rangle$. Let $\alpha_{1}, \ldots, \alpha_{k}$ be those coatoms of $\operatorname{Co}(\mathbf{F})$ for which $\gamma_{j} \leqslant \alpha_{i}$ for some $j=1, \ldots, m$. We let $\delta$ denote $\Lambda_{i=1}^{k} \alpha_{i}$ and $\mathbf{A}_{i}=\mathbf{F} / \alpha_{i} \in(H(\mathscr{K}))_{S}$. From condition (ii) we conclude $\mathbf{F} / \delta=\mathbf{A}_{1} \times \cdots \times \mathbf{A}_{k}$.

If $s \in F$ with $s / \alpha_{i}=1_{A_{i}}$ for some $i$, and if $\gamma_{j} \leqslant \alpha_{i}$, then $s / \gamma_{j} \equiv 1_{C_{j}}\left(\alpha_{i} / \gamma_{j}\right)$. Hence $s^{\prime} / \gamma_{j}=1_{C_{j}}$. A similar statement is true for $0_{A_{i}}$. Since $\mathbf{F} / \delta=\mathbf{A}_{1} \times \cdots \times \mathbf{A}_{k}$, for any $e=\left\langle e_{1}, \ldots, e_{k}\right\rangle$ with $e_{i} \in\left\{0_{A_{i}}, 1_{A_{i}}\right\}$, the element $e$ is in $\mathbf{F} / \delta$ and hence for some $s \in F, s / \delta=e$. Let $s^{\prime}=s \oplus 0_{F} \in F$. Then for all $\gamma_{j} \leqslant \alpha_{i}, s^{\prime} / \gamma_{j}=1_{C_{j}}$ if $e_{i}=1_{A_{i}}$ and $s^{\prime} / \gamma_{j}=0_{C_{j}}$ if $e_{i}=0_{A_{i}}$. Therefore, the following are all elements of $F$ : $0_{F}, 1_{F}$, and $s_{i}, i=1, \ldots, k$, where $g\left(1_{F}\right)=\left\langle 1_{C_{1}}, \ldots, 1_{C_{m}}\right\rangle, g\left(0_{F}\right)=\left\langle 0_{C_{1}}, \ldots, 0_{C_{m}}\right\rangle$, and $g\left(s_{i}\right)=\left\langle c_{1}, \ldots, c_{m}\right\rangle$ with $c_{j}=1_{C_{j}}$ if $\gamma_{j} \leqslant \alpha_{i}$ and $c_{j}=0_{C_{j}}$ if $\gamma_{j} \nless \alpha_{i}$.

$\mathscr{V}$ has FHP by condition (iv) and Lemma 2.2. Moreover, for every finite algebra $\mathbf{B} \in \mathscr{V}$, there exist $1_{B}$ and $0_{B}$ in $B$ for which the equations of condition (iv) hold since $\mathbf{B}$ is a homomorphic image of $\mathbf{F}_{\mathscr{V}}(n)$ for some $n$. In particular if $\mathbf{F} / \boldsymbol{\theta}=\mathbf{B}$, then $1_{F} / \theta=1_{F / \theta}=1_{B}$.

For each $i, 1 \leqslant i \leqslant k$, define $\beta_{i}=\wedge\left\{\gamma_{j} \mid \gamma_{j} \leqslant \alpha_{i}, 1 \leqslant j \leqslant m\right\}$. F is a subdirect product of the $\mathbf{F} / \beta_{i}$ since $\Lambda_{1 \leqslant i \leqslant k} \beta_{i}=\Lambda_{1 \leqslant j \leqslant m} \gamma_{j}=\Delta_{F}$. Let $\mathbf{B}_{i}=\mathbf{F} / \beta_{i}$. For each $i$, $1 \leqslant i \leqslant k$, let $b_{i} \in B_{i}$ be arbitrary and let $a_{i} \in F$ be such that $a_{i} / \beta_{i}=b_{i}$. We consider $a \in F$ given by

$$
a=\left(\cdots\left(\left(\left(s_{1} \cdot a_{1}\right)+\left(s_{2} \cdot a_{2}\right)\right)+\left(s_{3} \cdot a_{3}\right)\right)+\cdots\right)+\left(s_{k} \cdot a_{k}\right) .
$$

A computation using (iv) shows that for each $i, 1 \leqslant i \leqslant k, a / \beta_{i}=b_{i}$. Therefore $\mathbf{F} \cong \mathbf{B}_{1} \times \cdots \times \mathbf{B}_{k}$ and $\left\{\beta_{1}, \ldots, \beta_{k}\right\}$ forms a factorizing set for $\mathbf{F}$. For each $i$, $\beta_{i} \leqslant \alpha_{i}$ and $\beta_{i} \nless \alpha_{j}$ for $j \neq i$ since $\beta_{i} \circ \Lambda_{r \neq i} \beta_{r}=\nabla_{F}$.

We complete the proof by showing that $\alpha_{1}, \ldots, \alpha_{k}$ are the only coatoms of $\operatorname{Co}(\mathbf{F})$ and by appealing to Corollary 3.9. So let $\alpha \in \operatorname{Co}(\mathbf{F})$ be a coatom with $\alpha \neq \alpha_{i}$, $i=1, \ldots, k$. By FHP $\alpha=\left(\alpha \vee \beta_{1}\right) \wedge \cdots \wedge\left(\alpha \vee \beta_{k}\right)$. Hence for some $i$, say $i=1$, $\alpha=\alpha \vee \beta_{1}$ and thus $\alpha \geqslant \beta_{1}$. Since $\alpha_{1} \vee \alpha=\nabla_{F}$, there exist $a, b \in F$ with $a \equiv b(\alpha)$ and $a \not \equiv b\left(\alpha_{1}\right)$. If $\mathbf{A}$ is any simple algebra in $H(\mathscr{K})$ and if $x, y \in A$, $x \neq y$, then by (vi) there is a unary polynomial $p$ with $\{p(x), p(y)\}=\left\{0_{A}, 1_{A}\right\}$. Since $\mathbf{F} / \alpha_{1} \in H(\mathbf{K})_{S}$, there is a polynomial $p$ such that, say, $p(a) \equiv 1_{F}\left(\alpha_{1}\right)$ and $p(b) \equiv 0_{F}\left(\alpha_{1}\right)$. Since $0_{F} / \gamma_{i}=0_{C_{i}}$ and $1_{F} / \gamma_{i}=1_{C_{i}}$ we get for all $\gamma_{i} \leqslant \alpha_{1}, p(a) / \gamma_{i} \equiv$ $1_{F} / \gamma_{i}=1_{C_{i}}\left(\alpha_{1} / \gamma_{i}\right)$ and $p(b) / \gamma_{i} \equiv 0_{F} / \gamma_{i}=0_{C_{i}}\left(\alpha_{1} / \gamma_{i}\right)$. We apply condition (v) to give $p(a)^{\prime} / \gamma_{i}=1_{F} / \gamma_{i}$ and $p(b)^{\prime} / \gamma_{i}=0_{F} / \gamma_{i}$. This gives $p(a)^{\prime} \equiv 1_{F}\left(\beta_{1}\right)$ and $p(b)^{\prime}$ $\equiv 0_{F}\left(\beta_{1}\right)$ since $\beta_{1}=\wedge\left\{\gamma_{i} \mid \gamma_{i} \leqslant \alpha_{1}\right\}$. So $0_{F} \equiv 1_{F}(\alpha)$ since $\beta_{1}<\alpha$ and $p(a)^{\prime} \equiv$ $p(b)^{\prime}(\alpha)$. If $0_{F} \equiv 1_{F}(\alpha)$, then $\alpha=\nabla_{F}$ contradicting $\beta \leqslant \alpha<\nabla_{F}$. 
An obvious candidate for $\mathscr{K}$ is $\mathscr{V}_{F S I}$. However, in order to apply Theorem 5.2 in this case would require knowledge of the family of all subdirectly irreducible algebras in $\mathscr{V}$. This knowledge is often difficult to obtain, even for finitely generated varieties except if they are congruence distributive. The next corollary provides a more readily applicable choice of $\mathscr{K}$ for finitely generated $\mathscr{V}$.

COROLlaRY 5.3. Let $\mathbf{B}$ be a finite algebra with $\mathscr{V}=H S P(\mathbf{B})$ and $\mathscr{K}=(H S(\mathbf{B}))_{S I}$. Suppose $\mathscr{V}$ has binary terms,$+ \cdot \cdot$, and $\oplus$ for which (ii), (iii), (iv), (v), and (vi) of Theorem 5.2 hold for $\mathscr{V}$ and for this choice of $\mathscr{K}$. Then $\mathscr{V}$ has FHAP and $\mathscr{V}_{F S} \subseteq H S(\mathbf{B})$.

Proof. For each $n<\omega, \mathbf{F}_{\mathscr{V}}(n)$ is a subdirect product of members of $S(\mathbf{B})$. Each $\mathbf{A} \in S(\mathbf{B})$ is a subdirect product of subdirectly irreducibles and each irreducible is in $H(\mathbf{A})$. So $\mathbf{F}_{\mathscr{V}}(n) \in P_{S D}\left((H S(\mathbf{B}))_{S I}\right)$ and Theorem 5.2 applies.

REMARK 5.4. In our applications of Theorem 5.2 and Corollary 5.3 we frequently have algebras with lattice reducts in which meet and join provide obvious candidates for - and + . The operation $x \oplus y$ need not explicitly involve the variable $y$ and in some cases, when $0_{A} / \alpha$ and $1_{A} / \alpha$ are singleton sets for all $\mathbf{A} \in \mathscr{K}, x \oplus y=x$ may be used. Condition (vi) is automatically satisfied if all algebras in $H(\mathscr{K})_{S}$ have exactly two elements. Also if each algebra in $H(\mathscr{K})_{S}$ generates a congruence permutable variety, then by Lakser [1982], condition (vi) holds for $\mathscr{K}$. Condition (vi) is a special case of a condition considered in Burris and Lawrence [1981].

The varieties presented in Examples 1.1, 1.2 and 1.4 are easily seen to satisfy the hypotheses of Corollary 5.3. The meet and join operations serve for $\cdot$ and + . In the example of Stone algebras we take for $\mathscr{K}=\{\mathbf{S}, \mathbf{Q}\}$, where $\mathbf{S}$ is the 3-element Stone algebra, $\mathbf{Q}$ the 2-element Boolean algebra. Then conditions (i)-(iv) are met, and by choosing $x^{\prime}=x^{* *},(\mathrm{v})$ is satisfied as well. $\mathbf{Q}$ is the only simple, so (vi) is immediate. In Example 1.2 we have $\mathscr{K}=\left\{\mathbf{B}_{j} \mid 1 \leqslant j \leqslant k\right\}$, and $x^{\prime}=\left(x \rightarrow 0_{A}\right) \rightarrow 0_{A}$. Similarly, in Example 1.4 we choose $\mathscr{K}=\left\{\mathbf{I}_{j} \mid 1 \leqslant j \leqslant k\right\}$ and $x^{\prime}=\square\left(\square x \Rightarrow 0_{A}\right) \Rightarrow 0_{A}$. Finally, in Example 1.3 we let $\mathscr{K}=\{\mathbf{A}, \mathbf{A} / \alpha\}$. By the very definition of the algebra $\mathbf{A}$, there are terms $+, \cdot,{ }^{\prime}, 0,1$ which behave as desired on $\mathbf{A}$, and therefore on $\mathbf{A} / \alpha$ as well, and hence the corollary can be applied.

All the examples given so far have been congruence distributive. We will see examples of varieties with FHAP which fail to satisfy any congruence identities (\$6). A natural example of a variety with FHAP which fails to be congruence distributive is furnished by the varieties of rings with unit generated by $\mathbf{Z}_{p^{k}}=$ $\left\langle Z /\left(p^{k}\right),+, \cdot, 0,1\right\rangle$ for some prime $p$ and $k \geqslant 1$. We finish this section with a brief discussion of these varieties.

EXAMPLE 5.5. Let $\mathscr{V}=H S P\left(\mathbf{Z}_{p^{k}}\right), k \geqslant 1$. Note that $H S\left(\mathbf{Z}_{p^{k}}\right)=H\left(\mathbf{Z}_{p^{k}}\right)=$ $I\left\{\mathbf{Z}_{p^{\prime}} \mid 0 \leqslant j \leqslant k\right\}$. Let $\mathscr{K}=\left\{\mathbf{Z}_{p^{j}} \mid 1 \leqslant j \leqslant k\right\}$. We verify that conditions (ii)-(vi) of Corollary 5.3 are satisfied.

(ii) $(H(\mathscr{K}))_{S}=\left\{\mathbf{Z}_{p}\right\}$, which is a multiplicative class, as $H S P\left(\mathbf{Z}_{p}\right)$ is congruence permutable. Hence (vi) also holds by Remark 5.4.

(iii) $\left(\operatorname{Co}\left(\mathbf{Z}_{p^{j}}\right), \subseteq\right)$ is a chain of length $j$. The unique coatom $\alpha_{j}$ is the congruence corresponding to the ideal generated by $p^{j-1}$. 
(iv) $+, \cdot, 0,1$ are taken to be the usual ring operations.

(v) The operation $x^{\prime}=x^{p}$ satisfies the requirements: If $a \in 0 / \alpha_{j}$, then $a=i p^{j-1}$ $\bmod p^{j}$, and hence $a^{p}=i^{p} p^{j} \bmod p^{j}=0 \bmod p^{j}$. If $a \in 1 / \alpha_{j}$, then $a=1+i p^{j-1}$ $\bmod p^{j}$, and hence $a^{p}=\left(1+i p^{j-1}\right)^{p} \bmod p^{j}=1 \bmod p^{j}$. Hence $\mathscr{V}$ has FHAP.

6. Derived varieties. Let $\mathscr{V}$ be a locally finite variety with FHAP. We will associate with $\mathscr{V}$ a family $d(\mathscr{V})$ of varieties which we call the derived varieties of $\mathscr{V}$. In the next section we will investigate the relationships between the finitely generated free algebras in $\mathscr{V}$ on the one hand, and those in the varieties $d(\mathscr{V})$ and $\mathscr{V}_{0}$ on the other hand.

Some terminology from category theory will be helpful in the following.

Given a concrete category $\mathscr{C}$ and an object $\mathbf{Q}$ in $\mathscr{C}$, we will denote by $\mathscr{C} \downarrow \mathbf{Q}$ the category whose objects are pairs $(\mathbf{A}, f)$, where $\mathbf{A}$ is an object of $\mathscr{C}, f: \mathbf{A} \rightarrow \mathbf{Q}$ a surjective morphism, and whose morphisms are arrows $h:(\mathbf{A}, f) \rightarrow(\mathbf{B}, g)$ such that $h: \mathbf{A} \rightarrow \mathbf{B}$ is a $\mathscr{C}$-morphism and $g \circ h=f:$

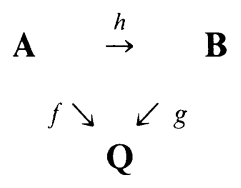

(cf. Mac Lane [1971, p. 46]).

Of particular importance to us will be the category $\mathscr{V}_{F D I} \downarrow \mathbf{Q}$, with $\mathbf{Q} \in \mathscr{V}_{F S}$. Observe that if $(\mathbf{A}, f)$ and $\left(\mathbf{A}, f^{\prime}\right)$ are $\mathscr{V}_{F D I} \downarrow \mathbf{Q}$-objects, then $f^{\prime}=f \circ g$ for some isomorphism $g: \mathbf{Q} \rightarrow \mathbf{Q}$. We will sometimes denote an object $(\mathbf{A}, f) \in \mathscr{V}_{F D I} \downarrow \mathbf{Q}$ by its algebra part $\mathbf{A}$. Note that $\mathbf{A} \in \mathscr{V}_{F D I} \downarrow \mathbf{Q}$ if and only if $\mathbf{A} / \alpha \cong \mathbf{Q}$, where $\alpha$ is the unique coatom of $\operatorname{Co}(\mathbf{A})$. If $\mathscr{V}_{F S}=\left\{\mathbf{Q}_{1}, \ldots, \mathbf{Q}_{k}\right\}$, with $\mathbf{Q}_{i} \not \mathbf{Q}_{j}, i \neq j$, then $\mathscr{V}_{F D I}=$ $\bigcup_{i=1}^{k} \mathscr{V}_{F D I} \downarrow \mathbf{Q}_{i}$, and the union is disjoint.

Suppose $\mathscr{V}$ has similarity type $\tau$. For $\mathbf{Q} \in \mathscr{V}_{F S}$ and $q \in Q$ let $\tau_{q}$ be the similarity type consisting of all terms $t$ of type $\tau$ which satisfy $t^{\mathbf{Q}}(q, \ldots, q)=q$. For $(\mathbf{A}, f) \in \mathscr{V}_{F D I} \downarrow \mathbf{Q}$ and $q \in \mathbf{Q}$ let $(\mathbf{A}, f)_{q}$ be the algebra of type $\tau_{q}$ with domain $A_{q}=f^{-1}(\{q\})$ :

$$
(\mathbf{A}, f)_{q}=\left\langle A_{q},\left\langle\left. t\right|_{A_{q}^{n}}: t \text { an } n \text {-ary } \mathscr{V} \text {-term, } t^{\mathbf{Q}}(q, \ldots, q)=q, n<\omega\right\rangle\right\rangle .
$$

If no confusion is likely, we will write $\mathbf{A}_{q}$ for $(\mathbf{A}, f)_{q}$. Observe that if $(\mathbf{A}, f)$, $(\mathbf{A}, g) \in \mathscr{V}_{F D I} \downarrow \mathbf{Q}$, then there is an isomoprhism $h:(\mathbf{A}, f) \rightarrow(\mathbf{A}, g)$ and hence $(\mathbf{A}, f)_{q}=(\mathbf{A}, g)_{q^{\prime}}$ for some $q^{\prime} \in Q$. Hence the collection $\left\{(\mathbf{A}, f)_{q}: q \in Q\right\}$ is independent of the choice of $f$. For $\mathbf{Q} \in \mathscr{V}_{F S}$ and $q \in Q$ let

$$
d(\mathscr{V})_{q}=\operatorname{HSP}\left\{(\mathbf{A}, f)_{q} \mid(\mathbf{A}, f) \in \mathscr{V}_{F D I} \downarrow \mathbf{Q}\right\} .
$$

The variety $d(\mathscr{V})_{q}$ is called a derived variety of $\mathscr{V}$. If $(\mathbf{A}, f) \in \mathscr{V}_{F D I} \downarrow \mathbf{Q}$, then the algebras $(\mathbf{A}, f)_{q}$ yield a natural decomposition of $\mathbf{A}$ as a union of $d(\mathscr{V})_{q}$-algebras.

If $\mathscr{V}_{F S}=\left\{\mathbf{Q}_{1}, \ldots, \mathbf{Q}_{k}\right\}$, then we let

$$
d(\mathscr{V})=\left\langle d(\mathscr{V})_{q} \mid q \in Q_{i}, i=1, \ldots, k\right\rangle .
$$

Our first objective it to obtain a more manageable description of the varieties $d(\mathscr{V})_{q}$. 
LEMMA 6.1. Let $(\mathbf{A}, f), \mathbf{B} \in \mathscr{V}_{F D I} \downarrow \mathbf{Q}$, and suppose there exists a homomorphism $h$ from $\mathbf{B}$ onto $\mathbf{A}$. Then for any $q \in Q, \mathbf{A}_{q}$ is a $\tau_{q}$-homomorphic image of some $\mathbf{B}_{q}$.

Proof. Let $k=f \circ h: \mathbf{B} \rightarrow \mathbf{Q}$, then $(\mathbf{B}, k) \in \mathscr{V}_{F D I} \downarrow \mathbf{Q}$, and $h:(\mathbf{B}, k) \rightarrow(\mathbf{A}, f)$. Hence, for $q \in \mathbf{Q}, h\left((\mathbf{B}, k)_{q}\right)=(\mathbf{A}, f)_{q}$.

Lemma 6.2. Let $(\mathbf{A}, f) \in \mathscr{V}_{F D I} \downarrow \mathbf{Q}$, and $\mathbf{B}_{i}, i \in I$ in $\mathscr{V}_{F D I}$, with $\mathbf{A}$ a subdirect product of the $\mathbf{B}_{i}, i \in I$. Then $\mathbf{B}_{i} \in \mathscr{V}_{F D I} \downarrow \mathbf{Q}$ as well, and for any $q \in Q, \mathbf{A}_{q}$ is a subdirect product of the family $\left(\mathbf{B}_{i}\right)_{q}, i \in I$.

Proof. Let $f_{i}: \mathbf{A} \rightarrow \mathbf{B}_{i}, i \in I$, be onto homomorphisms with $\bigwedge_{i \in I} \operatorname{ker} f_{i}=\Delta_{A}$. The congruence $\operatorname{ker} f$ is a unique coatom in $\operatorname{Co} \mathbf{A}$, and $\operatorname{ker} f_{i} \leqslant \operatorname{ker} f, i \in I$, hence there exist onto homomorphisms $g_{i}: \mathbf{B}_{i} \rightarrow \mathbf{Q}$, and morphisms $p_{i}:(\mathbf{A}, f) \rightarrow\left(\mathbf{B}_{i}, g_{i}\right)$. Fix $q \in Q$, and let $\left.p_{i}\right|_{A_{q}}: \mathbf{A}_{q} \rightarrow\left(\mathbf{B}_{i}\right)_{q}$. Then $\left.p_{i}\right|_{A_{q}}$ is an onto $\tau_{q}$-homomorphism, and $\left.\Lambda_{i \in I} \operatorname{ker} p_{i}\right|_{A_{q}}=\Delta_{A_{q}}$. Thus $\mathbf{A}_{q}$ is a subdirect product of the algebras $\left(\mathbf{B}_{i}\right)_{q}, i \in I$.

THEOREM 6.3. Let $\mathscr{V}$ be a locally finite variety having FHAP and let $\mathbf{Q} \in \mathscr{V}_{F S}$ with $q \in Q$.

(i) $d(\mathscr{V})_{q}=H S P\left\{\mathbf{A}_{q} \mid \mathbf{A} \in \mathscr{V}_{F S I}\right\}$.

(ii) $d(\mathscr{V})_{q}=H S P\left\{\mathbf{A}_{q} \mid \mathbf{A}\right.$ is a directly indecomposable factor of $\left.\mathbf{F}_{\mathscr{V}}(n), n<\omega\right\}$.

(iii) If $\mathscr{V}=\operatorname{HSP}(\mathscr{K})$ for a set of finite algebras $\mathscr{K}$, then $d(\mathscr{V})_{q}=\operatorname{HSP}\left\{\mathbf{A}_{q} \mid \mathbf{A} \in\right.$ $\left.(H S(\mathscr{K}))_{S I}\right\}$. In particular, if $\mathscr{V}$ is finitely generated then so is $d(\mathscr{V})_{q}$.

Proof. (i) Follows from Lemma 6.2. For (ii), each $\mathbf{B} \in \mathscr{V}_{F D I}$ is a homomorphic image of $\mathbf{F}_{\mathscr{\gamma}}(n)$ for some finite $n$. If $\mathbf{F}_{\mathscr{\gamma}}(n) / \gamma \cong \mathbf{B}$ and if $\alpha$ is the unique coatom of $\operatorname{Co}\left(\mathbf{F}_{\mathscr{V}}(n)\right)$ above $\gamma$, then by Theorem $3.4, \alpha^{++} \leqslant \gamma \leqslant \alpha$. The algebra $\mathbf{F}_{\mathscr{\gamma}}(n) / \alpha^{++}$is a directly indecomposable factor of $\mathbf{F}_{\mathscr{V}}(n)$. Lemma 6.1 gives $\mathbf{B}_{q} \in H\left(\left(\mathbf{F}_{\mathscr{V}}(n) / \alpha^{++}\right)_{q}\right)$. In order to prove (iii), by Lemma 6.2 and (ii), it suffices to show that if $\mathbf{A}$ is a directly indecomposable factor of $\mathbf{F}_{\mathscr{V}}(n), n$ finite, then $\mathbf{A}$ is a subdirect product of members of $(H S(\mathbf{B}))_{S I}$. But this follows from Lemma 4.5 , since clearly $\mathbf{F}_{\mathscr{V}}(n) \in$ $P_{S}\left((H S(\mathbf{B}))_{S I}\right)$.

Given $\mathbf{A}$ of similarity type $\tau$ with $\mathbf{A} / \alpha \cong \mathbf{Q} \in \mathscr{V}_{F S}$, for $q \in Q$ we wish to describe a method for determining a generating set for the operations of the algebra

$$
\mathbf{A}_{q}=\left\langle A_{q},\left\langle\left. t\right|_{A_{q}^{n}}: t \text { an } n \text {-ary } \tau \text {-term, } t^{\mathbf{Q}}(q, \ldots, q)=q, n<\omega\right\rangle\right\rangle .
$$

For $\left\langle q, q^{\prime}\right\rangle \in Q^{2}$ we let $T_{q q^{\prime}}$ denote all term operations $t$ of $\mathbf{A}$ for which $t\left(A_{q}, \ldots, A_{q}\right) \subseteq A_{q^{\prime}}$. Since $\alpha$ is a congruence relation, for any $t$ of type $\tau$ any any $q \in Q$, there is a $q^{\prime} \in Q$ with $t \in T_{q q^{\prime}}$. The set of operations $T_{q q}$ determines the operations of $\mathbf{A}_{q}$. We wish to find a small set $G_{q q} \subseteq T_{q q}$ such that $G_{q q}$ generates $T_{q q}$ (notation: $\left\langle G_{q q}\right\rangle=T_{q q}$ ). To this end we choose $G_{q q^{\prime}} \subseteq T_{q q^{\prime}}$, for each $q^{\prime} \in Q$, and we want to show each $G_{q q^{\prime}}$ generates $T_{q q^{\prime}}$ in the following sense:

$$
\begin{aligned}
& t\left(x_{1}, \ldots, x_{n}\right) \in T_{q q^{\prime}} \text { implies there exist } g\left(x_{1}, \ldots, x_{n}\right) \in G_{q q^{\prime}} \\
& \text { and } s_{1}\left(x_{1}, \ldots, x_{n}\right), \ldots, s_{k}\left(x_{1}, \ldots, x_{n}\right) \in\left\langle G_{q q}\right\rangle \\
& \text { with }\left.t\right|_{A_{q}^{n}}=\left.g\left(s_{1}, \ldots, s_{k}\right)\right|_{A_{q}^{n}}
\end{aligned}
$$


Typically for a given $q$ this verification is done as a routine simultaneous induction over all term operations $t$ of $\mathbf{A}$ and all the $T_{q q^{\prime}}$, the induction being done on the form of $t$.

For a concrete example consider the ring $\mathbf{Z}_{4}$ with unit having universe $\{0,1,2,3\}$. Let $\mathbf{Z}_{4} / \alpha$ be the 2-element ring with universe $\{0,1\}$. The classes of $\alpha$ are $A_{0}=\{0,2\}$ and $A_{1}=\{1,3\}$. We wish to determine $T_{00}$. Consider

$$
G_{00}=\left\{x_{1}, \ldots, x_{n}, x_{1}+x_{2}, 0,2\right\} \quad \text { and } G_{01}=\left\{1+x_{1}, 1,3\right\} .
$$

It is easily seen that $G_{00} \subseteq T_{00}$ and $G_{01} \subseteq T_{01}$. Consider $t=t\left(x_{1}, \ldots, x_{n}\right)$. If $t$ is a constant or a variable, then $t \in G_{00} \cup G_{01}$. Suppose $t=t_{1}+t_{2}$, for terms $t_{1}=$ $t_{1}\left(x_{1}, \ldots, x_{n}\right)$ and $t_{2}=t_{2}\left(x_{1}, \ldots, x_{n}\right)$. If $t \in T_{00}$, then either $t_{1}, t_{2} \in T_{00}$ or $t_{1}$, $t_{2} \in T_{01}$. In the first case, by induction $t_{1}, t_{2} \in\left\langle G_{00}\right\rangle$ so $t=t_{1}+t_{2} \in\left\langle G_{00}\right\rangle$ as well. In the second case $t_{1}, t_{2} \in\left\langle G_{01}\right\rangle$ so $t_{1}=1+s_{1}, t_{2}=1+s_{2}$ for some $s_{1}$, $s_{2} \in\left\langle G_{00}\right\rangle$. So $t=t_{1}+t_{2}=\left(1+s_{1}\right)+\left(1+s_{2}\right)=2+s_{1}+s_{2} \in\left\langle G_{00}\right\rangle$. A similar argument applies for $t=t_{1} \cdot t_{2}$. Note that $x_{1} \cdot x_{2}$ restricted to $A_{0}$ is constant.

In general, we have no algorithm for selecting sets $G_{q q^{\prime}}$; the inductive method we have just described is only a way of verifying that a given choice of $G_{q q^{\prime}}$ will suffice.

If $\left|A_{q}\right|=1$, then $\mathbf{A}_{q}$ generates the trivial variety. Many of our examples involve directly indecomposable algebras $\mathbf{A}$ in which all but one $\mathbf{A}_{q}$ is trivial. For such $\mathbf{A}$ we have the following method of constructing $G_{q q^{\prime}}$.

LEMma 6.4. Suppose $\mathbf{A}$ is such that for exactly one $q \in Q,\left|A_{q}\right|>1$ and $\left|A_{q^{\prime}}\right|=1$ for all $q^{\prime} \neq q$. If $T_{q q^{\prime}} \neq \varnothing$, then let $G_{q q^{\prime}}=\{g\}$ where $g$ is any unary term for $\mathbf{A}$ with $g\left(A_{q}\right)=A_{q^{\prime}}$. Let $G_{q q}$ consist of all variables and all $f\left(y_{1}, \ldots, y_{k}\right) \in T_{q q}$ where $f$ is $a$ fundamental operation of $\tau$ and each $y_{i}$ is either a variable or is $g \in G_{q q^{\prime}}$ for some $q \neq q^{\prime}$. Then $G_{q q^{\prime}}$ generates $T_{q q^{\prime}}$ for all $q^{\prime} \in Q$.

Proof. For $q^{\prime} \neq q, T_{q q^{\prime}}$ is either empty or consists of terms that restricted to $A_{q}$ are constant. So $\left\langle G_{q q^{\prime}}\right\rangle=T_{q q^{\prime}}$ in this case. Let $t\left(x_{1} \cdots x_{n}\right) \in T_{q q}$. Suppose $t\left(x_{1}, \ldots, x_{n}\right)$ is of the form $f\left(s_{1}\left(x_{1} \cdots x_{n}\right), \ldots, s_{k}\left(x_{1} \cdots x_{n}\right)\right)$, with $f$ a fundamental operation of $\tau$. If $s_{i} \in T_{q q^{\prime}}, q^{\prime} \neq q$, then $s_{i}$ may be replaced by the term $g \in G_{q q^{\prime}}$. If $s_{i} \in T_{q q}$, then by the induction hypothesis, $s_{i} \in\left\langle G_{q q}\right\rangle$. So $G_{q q}$ generates $T_{q q}$.

We conclude this section with a description of the varieties $d(\mathscr{V})$ for a number of example varieties.

For the variety $\mathscr{S}$ of Stone algebras, Example 1.1, the only simple Stone algebra is the 2-element Boolean algebra with universe $\{0,1\} . \mathscr{S}_{0}$, the prime variety of $\mathscr{S}$, is the variety of Boolean algebras. The only other subdirectly irreducible Stone algebra is the 3-element chain. Theorem 6.3(i) can be applied to find $d(\mathscr{S})$. The variety $d(\mathscr{S})_{0}$ is trivial since the congruence class $A_{0}$ is trivial in both subdirectly irreducibles. The variety $d(\mathscr{S})_{1}$ is the variety $\mathscr{D}_{1}$ of distributive lattices with unit. This can be seen using Lemma 6.4 on the 3-element chain: $G_{11}=\{x \wedge y, x \vee$ $y, 1, x, y\}$ and $G_{10}=\{0\}$.

In the variety $\mathscr{B}_{k}$ of Example 1.2 , the only simple algebra is the generalized Boolean algebra (without 0$) \mathbf{Q}$ with universe $\{0,1\}$. The variety $\mathscr{B}_{k}$ is generated by the $k$-element chain $\mathrm{C}$ with elements $a_{1}<a_{2}<\cdots<a_{k}=1$. We apply Theorem 
6.3(iii). The algebras in $(H S(\mathbf{C}))_{S I}$ are $j$-element chains $a_{k-j+1}, \ldots, a_{k-1}, a_{k}$, for $2 \leqslant j \leqslant k$. The congruence $\alpha$ on each such chain has classes $C_{0}=\left\{a_{k-j+1}\right\}$ and $C_{1}=\left\{a_{k-j+2}, \ldots, a_{k-1}, a_{k}\right\}$. Moreover, $C_{1}$ is the universe of a subalgebra of $\mathbf{C}$. It is easily verified that $d\left(\mathscr{B}_{k}\right)_{0}$ is the trivial variety and $d\left(\mathscr{B}_{k}\right)_{1}$ is $\mathscr{B}_{k-1}$.

For the variety $\mathscr{E}$ of Example 1.3 , the only simple algebra in $\mathscr{E}$ is a $k$-element primal algebra with universe $Q=\{1,2, \ldots, k\}$. The algebra $\mathbf{A}=\langle A, F\rangle$ generates the variety. If $A_{i}$ denotes the $i$ th congruence class of $\alpha$, then the algebra $\mathbf{A}_{i}$ is a primal algebra with $c_{i}$ elements. By Theorem 6.3(iii) $d(\mathscr{E})_{i}$ is the variety generated by $\mathbf{A}_{i}$.

The only simple algebra in the variety $\mathscr{I}_{k}, k \geqslant 1$, of Example 1.4 is the 2-element interior algebra $\mathbf{Q}$ on $\{0,1\}$, satisfying $\square 0=0, \square 1=1$. For any $j \geqslant 1,\left(\mathbf{I}_{j}\right)_{1}=\mathbf{I}_{j-1}^{-}$, $\left(\mathbf{I}_{j}\right)_{0}=\tilde{\mathbf{I}}_{j-1}^{-}$. Since $\left(H S\left(\mathbf{I}_{k}\right)\right)_{S I}=\left\{\mathbf{I}_{j}: 1 \leqslant j \leqslant k\right\}$, we find $d\left(\mathscr{I}_{k}\right)_{1}=\mathscr{I}_{k-1}^{-}, d\left(\mathscr{I}_{k}\right)_{0}$ $=\tilde{\mathscr{I}}_{k-1}$.

We present some additional examples of 3-element algebras that illustrate the ideas of this section and of $\S 5$. We consider algebras $\mathbf{B}$ with universe $B=\{a, b, c\}$. We represent binary operations on $B$ as $3 \times 3$ arrays and unary operations as $1 \times 3$ arrays. For example, if $B$ is ordered by $a<b<c$, then the meet operation would be

$\begin{array}{lll}a & a & a \\ a & b & b \\ a & b & c\end{array}$

EXAMPLE 6.5 (RACA [1969]). Let B have two binary operations

$$
+=\begin{array}{lll}
a & b & c \\
b & c & c \\
c & c & c
\end{array} \quad \text { and } \quad=\begin{array}{lll}
a & a & a \\
a & c & b \\
a & b & c
\end{array}
$$

and a unary operation ${ }^{*}=c \quad a \quad a$. The algebra $\mathbf{B}$ has $\{a, c\}$ as its only proper subuniverse. The only proper congruence on $\mathbf{B}$ is given by $\alpha=a \mid b c$ with $B / \alpha$ a Boolean algebra. So $H S(\mathbf{B})_{S}$ contains only the 2-element Boolean algebra $\mathbf{Q}$ with universe $\{0,1\}$. Here $a / \alpha$ corresponds to $0 \in Q$ and $b / \alpha=c / \alpha$ corresponds to $1 \in Q$. Corollary 5.3 may be applied with $\mathscr{K}=\{\mathbf{B}, \mathbf{Q}\}$, with $+, \cdot,^{\prime}$ being the operations $+, \cdot,^{* *}$, respectively, and $0_{B}=a, 1_{B}=c, 0_{Q}=0,1_{Q}=1$. So $\mathscr{V}=$ $H S P(\mathbf{B})$ has FHAP and $\mathscr{V}_{F S}=\{Q\}$. Theorem 6.3 and Lemma 6.4 can be used to show $d(\mathscr{V})_{0}$ is the trivial variety and $d\left(\mathscr{V}_{1}\right)$ is the variety of Boolean groups (i.e., the variety generated by the 2-element group).

EXAMPLE 6.6. Let $\mathbf{B}$ have two binary operations

$$
=\begin{array}{rrr}
a & a & a \\
a & b & b \\
a & b & c
\end{array} \text { and }+=\begin{array}{rrr}
a & b & c \\
b & c & c \\
c & c & c
\end{array}
$$

a unary operation ' $=c \quad a \quad a$, and constants $a, b$, and $c$. We will show that $\mathscr{V}=H S P(\mathbf{B})$ has FHAP and satisfies no nontrivial congruence identities. Corollary 5.3 may be used to show $\mathscr{V}$ does have FHAP. Indeed, the only simple algebra in $H S(\mathbf{B})$ is the Boolean algebra $\mathbf{Q}$ with universe $\{0,1\}$. For $\mathbf{B}$ the congruence $\alpha$ given by $a \mid b c$ has $B / \alpha \cong Q$ with $a / \alpha$ corresponding to $0, c / \alpha$ to 1 . Lemma 6.4 may be used to show $d(\mathscr{V})_{0}$ is the trivial variety and $d(\mathscr{V})_{1}$ is the variety of semilattices with both zero and unit. 
Let $n \geqslant 1$ be arbitrary and consider the algebra $\mathbf{B}^{n}$. Let $\bar{a}=\langle a, \ldots, a\rangle, \bar{c}=$ $\langle c, \ldots, c\rangle$, and $S=\{b, c\}^{n}$. Then $A=S \cup\{\bar{a}\}$ is the universe of a subalgebra $\mathbf{A}$ of $\mathbf{B}^{n}$. If $\gamma$ is any congruence on $\mathbf{A}$, and if $\bar{a} \equiv x(\gamma)$ for some $x \neq \bar{a}$, then $\bar{c}=a^{\prime} \equiv x^{\prime}$ $=\bar{a}(\gamma)$. But in $\mathbf{A}, \theta(\bar{a}, \bar{c})=\nabla_{A}$. So $\gamma=\nabla_{A}$. Let $\bar{\alpha}$ be the congruence on $\mathbf{A}$ with only two congruence classes: $S$ and $\{\bar{a}\}$. Then $\bar{\alpha}$ is the unique coatom of $\operatorname{Co}(\mathbf{A})$ and the interval $\left[\Delta_{A}, \bar{\alpha}\right]$ in $\operatorname{Co}(\mathbf{A})$ is isomorphic to the congruence lattice of the algebra $\mathbf{S}$ with universe $S$ and operations $\left.\cdot\right|_{S},+\left.\right|_{S}$, where $\mathbf{S}$ is isomorphic to the free meet-semilattice with unit on $n$ free generators. Freese and Nation [1973] have shown that the congruence lattices of semilattices satisfy no nontrivial lattice identity. We apply their result to the interval $\left[\Delta_{A}, \bar{\alpha}\right]$ to complete our proof.

Both Examples 6.5 and 6.6 as well as Example 1.1, Stone algebras, can be constructed by taking a 2-element Boolean algebra and replacing one element by an entire algebra and defining the operations on the new set in such a way that the hypotheses of Corollary 5.3 are satisfied. A more general construction is given in the next example.

EXAMPLE 6.7. For $m \geqslant 2$, let $\mathbf{Q}$ be the algebra with universe $\{0,1, \ldots, m-1\}$ and with binary operations of $\max$ and $\min$ and with unary operation $s$ given by $s(j)=j+1(\bmod m)$. So $\langle Q, \max , \min , s\rangle$ is the primal algebra as described by Post [1921]. For each $i, 0 \leqslant i<m$, let $\mathbf{A}_{i}$ be a finite algebra with two binary operations $\oplus$ and $\odot$ and a constant $c_{i} \in A_{i}$ such that $c_{0} \oplus a=a \oplus c_{0}=a$ for all $a \in A_{0}$ and $a \odot c_{m-1}={ }^{\prime} c_{m-1} \odot a=a$ for all $a \in A_{m-1}$. (Note that if $\mathbf{A}_{0}$ is the trivial algebra, then this condition on $\mathbf{A}_{0}$ is always satisfied.) Assume the $A_{i}$ are pairwise disjoint and let $A=\bigcup_{i} A_{i}$. We define binary operations + and $\cdot$ and a unary operation * on $A$ as follows: For arbitrary $a_{i} \in A_{i}$ and $a_{j} \in A_{j}, 0 \leqslant i, j<m$,

$$
\begin{array}{ll}
a_{i}+a_{j}=a_{i} \oplus a_{j} & \text { if } i=j, a_{\max \{i, j\}} \text { otherwise, } \\
a_{i} \cdot a_{j}=a_{i} \odot a_{j} & \text { if } i=j, a_{\min \{i, j\}} \text { otherwise, } \\
a_{i}^{*}=c_{s(i)} . &
\end{array}
$$

It is easily verified that $\operatorname{Co}(\mathbf{A})$ has exactly one coatom $\alpha$ and $\mathbf{A} / \alpha \cong \mathbf{Q}$. The set $\left\{c_{0}, c_{1}, \ldots, c_{m-1}\right\}$ is the universe of a subalgebra of every subalgebra of $\mathbf{A}$. Every subuniverse of $\mathbf{A}$ is of the form $C_{1} \cup C_{2} \cup \cdots \cup C_{m-1}$ with $C_{i} \subseteq A_{i}, c_{i} \in C_{i}$, and each $C_{i}$ the universe of a subalgebra of $\mathbf{A}_{i}$. Each subalgebra of $\mathbf{A}$ is also directly indecomposable and has a unique coatom in its congruence lattice. The hypotheses of Corollary 5.3 hold for $\mathscr{V}=H S P(\mathbf{A})$ using * iterated $m$ times for ' and $c_{0}$ for $0_{A}$ and $c_{m-1}$ for $1_{A}$. So $\mathscr{V}$ has FHAP. By Theorem 6.3(iii), $d(\mathscr{V})_{i}=\operatorname{HSP}\left(\mathbf{A}_{i}\right)$, $0 \leqslant i<m$.

EXAmPle 6.8 (RACA [1969]). Let $\mathbf{B}$ have universe $\{a, b, c\}$ and three binary operations

$$
\begin{array}{llllll}
a & b & c & a & a & a \\
b & a & a & a & b & b, \\
c & a & a & a & b & c
\end{array} \text { and } \begin{array}{lll}
a & a & a \\
a & c & b \\
a & b & c
\end{array}
$$

Let $\mathscr{V}$ denote the variety generated by $\mathbf{B}$. The only nontrivial congruence relation on $\mathbf{B}$ is $\alpha=a \mid b c$ with $\mathbf{B} / \alpha \cong \mathbf{Q}$, the 2-element Boolean ring with, say, universe $\{0,1\}$. Here 0 corresponds to $a / \alpha$ and 1 corresponds to $b / \alpha=c / \alpha$. A routine application 
of Corollary 5.3, Theorem 6.3, and Lemma 6.4 shows $\mathscr{V}$ has FHAP, $\mathbf{Q}$ is the only simple algebra in $\mathscr{V}$, and $d(\mathscr{V})_{0}$ is the trivial variety and $d(\mathscr{V})_{1}$ is the variety generted by the 2-element Boolean ring. Observe that the only difference between this example and the variety $\mathscr{B}_{3}$ of Example 1.2 (cf. remarks preceding Example 6.5) is that here the simple algebra $\mathbf{Q}$ is a Boolean ring with distinguished constant 0 , while there the simple algebra $\mathbf{Q}$ is a generalized Boolean algebra with distinguished constant 1 . Note that in both cases 0 corresponds to the 1-element class of $\alpha$, and 1 to the 2-element class of $\alpha$. In $\S 7$ we shall see how this seemingly minor difference produces a substantial distinction between their free algebras.

EXAMPLE 6.9. A more delicate 3-element example arises from complemented semigroups. For example, consider the algebra $\mathbf{B}$ with two binary operations

$$
.=\begin{array}{lll}
a & a & a \\
a & c & b \\
a & b & c
\end{array} \text { and } *=\begin{array}{rrr}
c & a & a \\
b & c & b . \\
c & c & c
\end{array}
$$

This is like Example 5 of Bosbach [1969, p. 286]. B has two proper nontrivial subalgebras, one with universe $C_{1}=\{a, c\}$ and the other with universe $C_{2}=\{b, c\}$. The subalgebras $\mathbf{C}_{1}$ and $\mathbf{C}_{2}$ are not isomorphic but they are polynomially equivalent. The only proper congruence on $\mathbf{B}$ is $\alpha=a \mid b c$ and $\mathbf{B} / \alpha$ is isomorphic to $\mathbf{C}_{1}$. So $H S(\mathbf{B})_{S}=\left\{\mathbf{C}_{1}, \mathbf{C}_{2}\right\}$. In order to apply Corollary 5.3 we have $\mathscr{K}=\left\{\mathbf{C}_{1}, \mathbf{C}_{2}, \mathbf{B}\right\}$. If the lattice order $a<b<c$ is imposed on $B$, then the meet operation for this lattice is given by $x \wedge y=(x * y) y$ and the join operation $x \vee y$ can be shown to be

$$
(((x * y) *(x y))((y * x) *(x y)))(((x x) y) *((x y)(y x))) \text {. }
$$

These operations $\vee$ and $\wedge$ may be used for + and $\cdot$ in condition (iv) of Theorem 5.2 with $1_{B}=1_{C_{1}}=1_{C_{2}}=c$ and $0_{B}=0_{C_{1}}=a$ and $0_{C_{2}}=b$. For condition (v), $x^{\prime}=0_{A} *\left(0_{A} * x\right)$ with $A=B, C_{1}$, or $C_{2}$ will suffice. So $\mathscr{V}=H S P(\mathbf{B})$ has FHAP. In order to find $d(\mathscr{V})$, let $\mathbf{Q}_{1} \cong \mathbf{C}_{1}$ and $\mathbf{Q}_{2} \cong \mathbf{C}_{2}$ with $\mathbf{Q}_{1}=\{0,1\}, Q_{2}=\{2,3\}$ and $h$ : $\mathbf{B} \rightarrow \mathbf{Q}_{1}$ given by $h(a)=0$ and $h(b)=h(c)=1$. Then Lemma 6.4 can be used to show that $d(\mathscr{V})_{1}$ is equivalent to the variety generated by the 2-element Boolean ring and that $d(\mathscr{V})_{0}, d(\mathscr{V})_{2}$, and $d(\mathscr{V})_{3}$ are each trivial varieties.

If $\mathbf{B}$ is an algebra satisfying the hypotheses of Corollary 5.3, then it is possible to augment the universe of $\mathbf{B}$ with additional elements and create a new algebra $\mathbf{C}$, such that $\mathbf{C}$ also satisfies the conditions of Corollary 5.3 and $B$ is a subuniverse or congruence class of $\mathbf{C}$. We present one construction of this type and we use it in Example 6.10 to show that there are finitely generated varieties $\mathscr{V}$, having FHAP, for which the prime variety $\mathscr{V}_{0}$ of $\mathscr{V}$ satisfies no nontrivial congruence identity.

Let $\mathbf{B}$ be an algebra satisfying the hypotheses of Corollary 5.3 and suppose $\mathbf{B}$ is directly indecomposable and $0_{B}$ and $1_{B}$ are constants in the similarity type of $\mathbf{B}$. Let $e \notin B$ and form the algebra $\mathbf{C}$ with universe $B \cup\{e\}$ and with operations all $f$ : $C^{n} \rightarrow C$ such that $\left.f\right|_{B^{n}}$ is an operation of $\mathbf{B}$. We will show that $\mathbf{C}$ is simple, $\mathbf{C}$ also satisfies the hypotheses of Corollary 5.3, and the variety generated by $\mathbf{B}$ is polynomially equivalent to a subvariety of the prime variety of $H S P(\mathbf{C})$.

It is immediate that $\mathbf{B}$ is polynomially equivalent to the subalgebra of $\mathbf{C}$ with universe $B$. Moreover, every proper subalgebra of $\mathbf{C}$ is polynomially equivalent to a subalgebra of $\mathbf{B}$. Let + and $\cdot$ on $\mathbf{C}$ be the extension of + and $\cdot$ of $\mathbf{B}$ given by 
$e+x=x+e=x, e \cdot x=x \cdot e=e$ for all $x \in C$. Then $1_{C}=1_{B}$ and $0_{C}=e$ for condition (iv) of Theorem 5.2. For each $b \in B$, let $+_{b}$ be the binary operation on $C$ defined by $e+{ }_{b} e=e+{ }_{b} b=e$ and $x+{ }_{b} y=1_{C}$ otherwise. Using these operations one can show that $\mathbf{C}$ is simple and that (iii), (v), and (vi) of Theorem 5.2 all hold for C. Since $\mathbf{A} \in H S(\mathbf{C})$ is polynomially equivalent to an algebra in $H S(\mathbf{B}) \cup\{\mathbf{C}\}$ it only remains to show $H S(\mathbf{C})_{S}$ is multiplicative in order to apply Corollary 5.3. Note that up until now all prime varieties we considered were arithmetical so verifying 5.2(ii) was always immediate in our earlier examples.

Let $\mathbf{D}$ be an irredundant subdirect product of $\mathbf{D}_{1}, \ldots, \mathbf{D}_{m}, \mathbf{D}_{i} \in H S(\mathbf{C})_{S}$, with $D \subseteq D_{1} \times \cdots \times D_{m}$. We will show $D=D_{1} \times \cdots \times D_{m}$. It suffices to show that for each $i, 1 \leqslant i \leqslant m$, there is an element $d_{i} \in D$ with $d_{i}(i)=1_{D_{i}}$ and $d_{i}(j)=0_{D_{j}}$ for $j \neq i$. The family $H(\mathbf{B})_{S}$ is multiplicative and $\mathbf{B}$ is polynomially equivalent to a subalgebra of $\mathbf{D}_{i}$ for those $i$ with $D_{i}=C$. By using the two unary operations $g_{0}, g_{1}$ defined by $g_{0}(e)=0_{B}, g_{0}(b)=1_{B}$ for all $b \in B, g_{1}(e)=1_{B}, g_{1}(b)=0_{B}$ for all $b \in B$, and the fact that $H S(\mathbf{B})_{S}$ is multiplicative, it is possible to show that there is an element $b_{i} \in D$ with $b_{i}(i)=1_{D_{i}}$ and for $j \neq i, b_{i}(j)=0_{B}$ if $D_{i}=C$ and $b_{i}(j)=0_{D_{i}}$ if $D_{i} \neq C$. By multiplying all elements in $D$ by $\cdot$ we get $0_{D} \in D$ with $0_{D}(i)=0_{D_{i}}$ for all $i$. Finally let $\oplus$ be any binary operation with $\left.\oplus\right|_{B^{2}}=+$, $0_{B} \oplus e=e, 1_{B} \oplus e=1_{B}$. Then $d_{i}=b_{i} \oplus 0_{D}$.

EXAMPLE 6.10. Let $\mathbf{B}=\left\langle\{a, b, c\},+, \cdot{ }^{\prime}, a, b, c\right\rangle$ be the algebra of Example 6.6. Let $\mathbf{C}$ be the algebra with universe $B \cup\{e\}$ constructed above and $\mathscr{V}=H S P(\mathbf{C})$. Then $\mathscr{V}$ has FHAP and $(\mathscr{V})_{0}$ contains $\mathbf{C}$ which has a subalgebra $\mathbf{B}^{\prime}$ with universe $\{a, b, c\}$ that is polynomially equivalent to $\mathbf{B}$. The variety $\operatorname{HSP}\left(\mathbf{B}^{\prime}\right)$ satisfies no nontrivial congruence identity. Hence $\mathscr{V}_{0}$ satisfies no nontrivial congruence identity. In particular, $\mathscr{V}_{0}$ is not arithmetical.

7. Free algebras in varieties with FHAP. We will now examine the relationship between the structure of the finitely generated free algebras in a locally finite variety $\mathscr{V}$ with FHAP on the one side, and that of the free algebras in the prime and derived varieties on the other. Information on the number of direct factors of the free algebra in $\mathscr{V}$ can often be obtained from the prime variety $\mathscr{V}_{0}$, while the structure of the direct factors is, to some degree, determined by the derived varieties.

In Theorem 4.3 we showed that if $\mathscr{V}$ is a locally finite variety with FHAP, which, in addition, satisfies $S\left(\mathscr{V}_{F S}\right) \subseteq P\left(\mathscr{V}_{S}\right)$, then $\mathscr{V}_{0}$ is arithmetical and semisimple. In this case, then, $\mathbf{F}_{\mathscr{V}_{0}}(n)$ is a direct product of simple algebras.

LEMMA 7.1. Let $\mathscr{V}$ be a locally finite variety with FHAP, satisfiying $S\left(\mathscr{V}_{F S}\right) \subseteq$ $P\left(\mathscr{V}_{S}\right)$. Let $\alpha_{1}, \ldots, \alpha_{k}$ be a list of the coatoms of $\mathbf{F}_{\mathscr{V}}(n)$. Then $\mathbf{F}_{\mathscr{V}_{0}}(n) \cong$ $\prod_{i=1}^{k} \mathbf{F}_{\mathscr{V}}(n) / \alpha_{i}$.

Proof. Let $\delta=\bigwedge_{i=1}^{k} \alpha_{i}$, and let $\left\{x_{1}, \ldots, x_{n}\right\}$ be a set of free generators of $\mathbf{F}_{\mathscr{V}}(n)$. Then $x_{1} / \delta, \ldots, x_{n} / \delta$ generate $\mathbf{F}_{\mathscr{V}}(n) / \delta$, and clearly $\mathbf{F}_{\mathscr{V}}(n) / \delta \in \mathscr{V}_{0}$. As $\mathscr{V}_{0}$ is semisimple, in order to show $\mathbf{F}_{\mathscr{V}}(n) / \delta$ is freely generated by $x_{1} / \delta, \ldots, x_{n} / \delta$ it is sufficient to show that any map $f:\left\{x_{1} / \delta, \ldots, x_{n} / \delta\right\} \rightarrow Q$ such that $\left[\left\{f\left(x_{1} / \delta\right), \ldots, f\left(x_{n} / \delta\right)\right\}\right]=\mathbf{Q}, \mathbf{Q} \in \mathscr{V}_{F S}$, can be extended to a homomorphism $\bar{f}$ : $\mathbf{F}_{\mathscr{r}}(n) / \delta \rightarrow \mathbf{Q}$. Let $g: \mathbf{F}_{\mathscr{V}}(n) \rightarrow \mathbf{Q}$ be the homomorphism satisfying $g\left(x_{i}\right)=f\left(x_{i} / \delta\right)$, 
$i=1, \ldots, n$. Then $g$ is onto, and hence $\operatorname{ker} g=\alpha_{i}$, where $1 \leqslant i \leqslant k$. Therefore $\operatorname{ker} g \geqslant \delta$, so by the homomorphism theorem there exists a homomorphism $\bar{f}$ : $\mathbf{F}_{\mathscr{V}}(n) / \delta \rightarrow \mathbf{Q}$ such that $\bar{f}(x / \delta)=g(x)$, for all $x \in \mathbf{F}_{\mathscr{V}}(n)$. $\bar{f}$ is the desired extension, and hence $\mathbf{F}_{\mathscr{V}_{0}}(n) \cong \mathbf{F}_{\mathscr{V}}(n) / \delta$. By Lemma 4.1, $\mathbf{F}_{\mathscr{V}}(n) / \delta \cong \prod_{i=1}^{k} \mathbf{F}_{\mathscr{V}}(n) / \alpha_{i}$, which completes the proof of the lemma.

COROLlaRY 7.2. Let $\mathscr{V}$ be a locally finite variety with FHAP satisfying $S\left(\mathscr{V}_{F S}\right) \subseteq$ $P\left(\mathscr{V}_{S}\right)$. Then the number of directly indecompsable factors of $\mathbf{F}_{\mathscr{V}_{0}}(n)$ equals that of $\mathbf{F}_{\mathscr{V}}(n)$.

Proof. Let $\alpha_{1}, \ldots, \boldsymbol{\alpha}_{k}$ be a list of the coatoms of $\mathbf{F}_{\mathscr{V}}(n)$. By Lemma 3.1 $\mathbf{F}_{\mathscr{V}}(n)$ has $k$ directly indecomposable factors, and by Lemma 7.1, so does $\mathbf{F}_{\mathscr{V}_{0}}(n)$.

If $\mathscr{V}_{0}=H S P(\mathbf{Q})$, with $\mathbf{Q}$ primal, then the number $k$ of direct factors of $\mathbf{F}_{\mathscr{V}_{0}}(n)$ is $|Q|^{n}$. If $\mathbf{Q}$ is quasiprimal, then $k$ can be determined from the structure of subalgebras and internal isomorphisms of $Q$. See Foster and Pixley [1964] and Quackenbush [1974] for examples. In Taylor [1975] all quasiprimal algebras $\mathbf{Q}$ with $Q=\{0,1\}$ are described in detail and their free algebras are explictly given. For the examples in this paper we only need observe that the free Boolean algebra on $n$ free generators has $2^{n}$ factors and the free Boolean ring has $2^{n}-1$.

We now turn our attention to the directly indecomposable factors of $\mathbf{F}_{\mathscr{V}}(n)$. From now on in this section, $\mathscr{V}$ will stand for a locally finite variety $\mathscr{V}$ with FHAP.

Let $X$ be any finite set, $\mathbf{Q} \in \mathscr{V}_{F S}$ and $f: X \rightarrow Q$ such that $[f(X)]=\mathbf{Q}$, and let $(\mathbf{B}, \bar{f}) \in \mathscr{V}_{F D I} \downarrow \mathbf{Q}, i:(X, f) \rightarrow(B, \bar{f})$, (i.e., $\left.\bar{f} \circ i=f\right)$. We say that $(\mathbf{B}, f)$ is freely generated by $(X, f)$ with respect to $i$ in $\mathscr{V}_{F D I} \downarrow \mathbf{Q}$ if for all $(\mathbf{C}, g) \in \mathscr{V}_{F D I} \downarrow \mathbf{Q}$ and for all $h:(X, f) \rightarrow(C, g)$ there exists a unique morphism $\bar{h}:(\mathbf{B}, \bar{f}) \rightarrow(\mathbf{C}, g)$ such that $\bar{h} \circ i=h$, or equivalently, such that $g \circ \bar{h}=\bar{f}$ (see Diagram A).

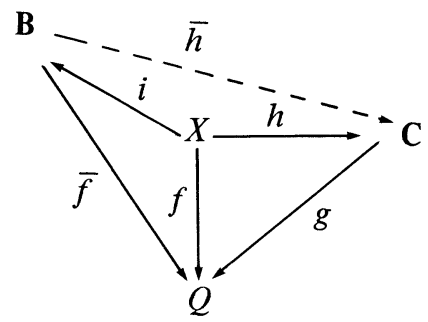

Diagram A

We often suppress the phrase "with respect to $i$ " and we always denote the homomorphism corresponding to $f$ by $f$. Observe that if $\left(\mathbf{B}_{1}, f_{1}\right)$ and $\left(\mathbf{B}_{2}, f_{2}\right)$ are both freely generated by $(X, f)$, then $\left(\mathbf{B}_{1}, f_{1}\right) \cong\left(\mathbf{B}_{2}, f_{2}\right)$, i.e., there exists an isomorphism $h: \mathbf{B}_{1} \rightarrow \mathbf{B}_{2}$ such that $f_{2} \circ h=f_{1}$.

We first establish the existence of free objects in $\mathscr{V}_{F D I} \downarrow \mathbf{Q}$.

THEOREM 7.3. Let $\mathscr{V}$ be a locally finite variety with FHAP and $\mathbf{Q} \in \mathscr{V}_{F S}$. Let $X$ be a finite set and $f: X \rightarrow Q$ such that $[f(X)]=\mathbf{Q}$. There is $a(\mathbf{B}, \bar{f}) \in \mathscr{V}_{F D I} \downarrow \mathbf{Q}$ which is freely generated by $(X, f)$ in $\mathscr{V}_{F D I} \downarrow \mathbf{Q}$. 
Proof. Let $\tilde{f}: \mathbf{F}_{\mathscr{V}}(X) \rightarrow Q$ be a homomorphism extending $f$, and $\alpha=\operatorname{ker} \tilde{f}$. Then $\alpha$ is a coatom of $\operatorname{Co}_{\mathscr{V}}(n)$, and by Theorem 3.4, $\mathbf{F}_{\mathscr{V}}(X) / \alpha^{++}$is directly indecomposable with $\alpha$ the unique coatom in $\left[\alpha^{++}, \nabla\right]$. Let $\bar{f}: \mathbf{F}_{\mathscr{\gamma}}(X) / \alpha^{++} \rightarrow \mathbf{Q}$ be such that $\bar{f} \circ \nu_{\alpha^{++}}=\tilde{f}$, and $i: X \rightarrow F_{\mathscr{V}}(X) / \alpha^{++}$given by $i(x)=x / \alpha^{++}$. Here $\nu_{\alpha^{++}}$ denotes the quotient map associated with $\alpha^{++}$. Then $i:(X, f) \rightarrow\left(F_{\mathscr{Y}}(X) / \alpha^{++}, \bar{f}\right)$. We claim that $\left(\mathbf{F}_{\mathscr{V}}(X) / \alpha^{++}, \bar{f}\right)$ is freely generated by $(X, f)$ in $\mathscr{V}_{F D I} \downarrow \mathbf{Q}$ with respect to $i$. To see this, let $(\mathbf{C}, g) \in \mathscr{V}_{F D I} \downarrow \mathbf{Q}$ and $h:(X, f) \rightarrow(C, g)$. Let $\tilde{h}$ : $\mathbf{F}_{\mathscr{V}}(X) \rightarrow \mathbf{C}$ extend $h: X \rightarrow C$. Then $g \circ \tilde{h}=\tilde{f}$, and hence $\operatorname{ker} \tilde{h} \leqslant \operatorname{ker} \tilde{f}=\alpha$. Again, by Theorem 3.4, we conclude ker $\tilde{h} \geqslant \alpha^{++}$. Hence there exists a homomorphism $\bar{h}$ : $\mathbf{F}_{\mathscr{V}}(X) / \alpha^{++} \rightarrow \mathbf{C}$ such that $\bar{h} \circ \nu_{\alpha^{++}}=\tilde{h}$. Since $\bar{h}(i(x))=\bar{h}\left(x / \alpha^{++}\right)=\bar{h} \circ \boldsymbol{\nu}_{\alpha^{++}}(x)=$ $\tilde{h}(x)=h(x)$, we have $\bar{h} \circ i=h$, as desired. Finally, the map $\bar{h}$ is unique since $i(X)$ generates $\mathbf{F}_{\mathscr{V}}(X) / \alpha^{++}$.

Given $\mathbf{Q} \in \mathscr{V}_{F S}$, a finite set $X$ and $f: X \rightarrow Q$ such that $[f(X)]=\mathbf{Q}$, let $\left(\mathbf{F}_{\mathscr{V}_{F D I} \downarrow Q}(X, f), \bar{f}\right)$ denote the object in $\mathscr{V}_{F D I} \downarrow \mathbf{Q}$ freely generated by $(X, f)$. We will call the algebra $F_{\mathscr{V}_{F D \downarrow} \downarrow \mathbf{Q}}(X, f)$ a free directly indecomposable.

COROllary 7.4. Let $\mathscr{V}$ be a locally finite variety with FHAP. Then $\mathbf{B} \in \mathscr{V}_{F}$ is a free directly indecomposable iff $\mathbf{B}$ is isomorphic to a directly indecomposable factor of $\mathbf{F}_{\mathscr{V}}(n)$, for some $n<\omega$.

Proof. $\Rightarrow$ Suppose $\left(\mathbf{B}, f^{*}\right)$ is freely generated by $(X, f)$ where $f: X \rightarrow Q$, $[f(X)]=\mathbf{Q}, X$ finite By the proof of 7.3, there is a directly indecomposable factor $\mathbf{F}_{\mathscr{V}}(X) / \alpha^{++}$of $\mathbf{F}_{\mathscr{V}}(X)$ and a map $\bar{f}: \mathbf{F}_{\mathscr{V}}(X) / \alpha^{++} \rightarrow \mathbf{Q}$ such that $\left(\mathbf{F}_{\mathscr{V}}(X) / \alpha^{++}, \bar{f}\right)$ is freely generated by $(X, f)$ as well. By the uniqueness of free objects in $\mathbf{V}_{F D I} \downarrow \mathbf{Q}$ we infer $\mathbf{B} \cong \mathbf{F}_{\mathscr{V}}(x) / \alpha^{++}$.

$\Leftarrow$ Let $\mathbf{B} \in \mathscr{V}_{F}$ be isomorphic to a directly indecomposable factor of $\mathbf{F}_{\mathscr{V}}(X), X$ finite. By the results of $\S 3, \mathbf{B} \cong \mathbf{F}_{\mathscr{V}}(X) / \alpha^{++}$for some coatom $\alpha$ of $\operatorname{Co}\left(\mathbf{F}_{\mathscr{V}}(X)\right)$. Let $\mathbf{Q}=\mathbf{F}_{\mathscr{V}}(X) / \alpha$ and $\tilde{f}: \mathbf{F}_{\mathscr{V}}(X) \rightarrow \mathbf{Q}, f=\tilde{f} \mid X$. Let $\bar{f}: \mathbf{F}_{\mathscr{V}}(X) / \alpha^{++} \rightarrow \mathbf{Q}$ be such that $\bar{f} \circ \nu_{\alpha^{++}}=\tilde{f}$. As in the proof of Theorem 7.3, one can verify that $\left(\mathbf{F}_{\mathscr{V}}(X) / \alpha^{++}, \bar{f}\right)$ is freely generated by $(X, f)$ in $\mathscr{V}_{F D I} \downarrow \mathbf{Q}$. Thus $\mathbf{B}$ is a free directly indecomposable.

By combining these results with Corollary 7.2 we get

COROLlary 7.5. Let $\mathscr{V}$ be a locally finite variety with FHAP such that $S\left(\mathscr{V}_{F S}\right) \subseteq$ $P\left(\mathscr{V}_{S}\right)$, and let $X$ be a finite set. Suppose $\mathbf{F}_{\mathscr{V}_{0}}(X)=\prod_{i=1}^{k} \mathbf{Q}_{i}$, where $\mathbf{Q}_{i} \in \mathscr{V}_{F S}$, $i=1, \ldots, k$, and let $f_{i}: X \rightarrow Q_{i}$ be $\left.p_{i}\right|_{X}$, where $p_{i}$ is the ith projection of $\mathbf{F}_{\mathscr{V}_{0}}(X)$ onto $\mathbf{Q}_{i}$. Then $\mathbf{F}_{\mathscr{V}}(X) \cong \prod_{i=1}^{k} \mathbf{F}_{\mathscr{\gamma}_{F D I} \downarrow \mathbf{Q}_{i}}\left(X, f_{i}\right)$.

We will now investigate the structure of the free indecomposables in $\mathscr{V}$. The results obtained apply to finitely generated free algebras in locally finite varieties with FHAP; since any such algebra belongs to a finitely generated variety with FHAP we will for the sake of simplicity from now on discuss such varieties only.

Suppose $\mathscr{V}=H S P(\mathbf{C})$, where $\mathbf{C}$ is a finite algebra, and $\mathscr{V}$ has FHAP. Let $\mathbf{B}=\mathbf{F}_{\mathscr{V}_{F D l} \downarrow \mathbf{Q}}(X, f)$, where $\mathbf{Q} \in \mathscr{V}_{F S}, X$ is a finite set and $f: X \rightarrow Q$ is a map such that $[f(X)]=\mathbf{Q}$. Since $\mathbf{F}_{\mathscr{V}}(X) \in P_{S D}\left(H S(\mathbf{C})_{S I}\right)$ and $\mathbf{B}$ is a direct factor of $\mathbf{F}_{\mathscr{V}}(X)$, it follows from Lemma 4.5 that $\mathbf{B} \in P_{S D}\left(H S(\mathbf{C})_{S I}\right)$ as well. In fact, if $\tilde{f}: \mathbf{F}_{\mathscr{V}}(X) \rightarrow \mathbf{Q}$ 
extends $f: X \rightarrow Q$, and $\alpha=\operatorname{ker} \tilde{f}$, then

$$
\mathbf{B}=\mathbf{F}_{\mathscr{V}}(X) / \alpha^{++} \in P_{S D}\left(\left\{\mathbf{F}_{\mathscr{V}}(X) / \gamma_{s}: s \in S\right\}\right)
$$

where $\alpha^{++} \leqslant \gamma_{s} \leqslant \alpha$ and $\mathbf{F}_{\mathscr{\gamma}}(X) / \gamma_{s} \in(H S(\mathbf{C}))_{S I}$. Let $\left\{\mathbf{C}_{s}: s \in S\right\}$ be the set of subdirectly irreducibles thus obtained, and $f_{s}: \mathbf{C}_{s} \rightarrow \mathbf{Q}$ the onto homomorphisms associated with $\gamma_{s}$. Then we have natural morphisms $p_{s}:(\mathbf{B}, \bar{f}) \rightarrow\left(\mathbf{C}_{s}, f_{s}\right)$ (in $\left.\mathscr{V}_{F D I} \downarrow \mathbf{Q}\right)$, and the family $\left\langle p_{s}: s \in S\right\rangle$ separates points. In fact, if we define the product $\prod_{s \in S}\left(\mathbf{C}_{s}, f_{s}\right)$ in $\mathscr{V} \downarrow \mathbf{Q}$ to be the pair $(\mathbf{P}, p)$, where $\mathbf{P}$ is the subalgebra of $\Pi_{s \in S} \mathbf{C}_{s}$ with universe

$$
\begin{aligned}
& P=\left\{\left(c_{s}\right)_{s \in S} \in \prod_{s \in S} C_{s} \mid f_{s}\left(c_{s}\right)=f_{s^{\prime}}\left(c_{s^{\prime}}\right), \text { all } s, s^{\prime} \in S\right\}, \\
& p: \mathbf{P} \rightarrow \mathbf{Q} \text { by } p\left(\left(c_{s}\right)_{s \in S}\right)=f_{s_{0}}\left(c_{s_{0}}\right) \text { for some } s_{0} \in S
\end{aligned}
$$

then $\prod_{s \in S} p_{s}:(\mathbf{B}, \bar{f}) \rightarrow \Pi_{s \in S}\left(\mathbf{C}_{s}, f_{s}\right)$ is a subdirect embedding in the category $\mathscr{V} \downarrow \mathbf{Q}$, (even in $\mathscr{V}_{F D} \downarrow \mathbf{Q}$ if $\Pi_{s \in S}\left(\mathbf{C}_{s}, f_{s}\right)$ is directly indecomposable). For future reference we collect these facts in the following theorem:

THEOREM 7.6. Let $\mathbf{C}$ be a finite algebra, and suppose the variety $\mathscr{V}=\operatorname{HSP}(\mathbf{C})$ has FHAP. Let $\mathbf{Q} \in \mathscr{V}_{F S}, X$ a finite set and $f: X \rightarrow Q$ such that $[f(X)]=\mathbf{Q}$. Then there exists a finite collection $\left\{\mathbf{C}_{s}: s \in S\right\} \subseteq(H S(\mathbf{C}))_{S I}$, onto homomorphisms $f_{s}: \mathbf{C}_{s} \rightarrow \mathbf{Q}$, $s \in S$, and $p_{s}: \mathbf{F}_{\mathscr{V}_{F D} \downarrow \mathbf{Q}}(X, f) \rightarrow \mathbf{C}_{s}$ such that $f_{s} \circ p_{s}=\bar{f}, s \in S$, and the family $\left\langle p_{s}: s \in S\right\rangle$ separates points.

These remarks lead to an obvious upperbound for $|B|$, which we will now compute in the special case that all subdirectly irreducibles in the representation of the free indecomposables are subobjects of a given object. This will be the case in almost all of our applications and will simplify notation considerably. Suppose $\mathscr{V}=\operatorname{HSP}(\mathbf{C}), \mathbf{Q} \in \mathscr{V}_{F S}$, and let us assume all subdirectly irreducibles $\left(\mathbf{C}_{s}, f_{s}\right)$ are embeddable in the fixed object $(\mathbf{D}, g) \in \mathscr{V}_{F D I} \downarrow \mathbf{Q}$. Then $(\mathbf{B}, f)$ can be embedded in $\Pi_{s \in S}(\mathbf{D}, g)$. Suppose $\mathbf{Q}$ has domain $\{1, \ldots, k\}$ and let, as usual, $D_{i}=g^{-1}(\{i\})$, $i=1, \ldots, k$, and suppose $\left|D_{i}\right|=c_{i}, i=1, \ldots, k$. Let $X_{i}=f^{-1}(\{i\}), i=1, \ldots, k$, $n_{i}=\left|X_{i}\right|, \quad i=1, \ldots, k$, and $n=|X|=\sum_{i=1}^{k} n_{i}$. We may assume that the representation of $(\mathbf{B}, \bar{f})$ is reduced, i.e., if $s \neq s^{\prime}$, then $\left.p_{s}\right|_{B} \neq\left. p_{s^{\prime}}\right|_{B}$, or, equivalently, $\left.p_{s} \circ i\right|_{X}$ $\neq\left. p_{s^{\prime}} \circ i\right|_{X}$. Since $p_{s} \circ i\left(X_{j}\right) \subseteq D_{j}$, it follows that $|S| \leqslant \prod_{i=1}^{k} c_{i}^{n_{i}}=m$. Also $|B|=$ $\sum_{i=1}^{k}\left|B_{i}\right|$, and $\left|B_{i}\right| \leqslant c_{i}^{|S|} \leqslant c_{i}^{m}$. Thus $|B| \leqslant \sum_{i=1}^{k} c_{i}^{m}$.

In Example 1.3 this upperbound is attained. As noticed in $\S 6$, the only simple algebra in $\mathscr{E}$ is the $k$-element primal algebra $\mathbf{Q}$. Let $g: \mathbf{A} \rightarrow \mathbf{Q}$. Given any $f: X \rightarrow Q$ let $H_{f}=\{h: X \rightarrow A \mid h:(X, f) \rightarrow(\mathbf{A}, g)\}$ and let $i: X \rightarrow \prod_{h \in H_{f}} A$ be the canonical map, that is $i(x)_{h}=h(x)$. It is easy to verify that $[i(X)] \cong \mathbf{F}_{\mathscr{E}_{F D l} \downarrow \mathbf{Q}}(X, f)$ and the universe of $[i(X)]$ is $\bigcup_{i=1}^{k} \prod_{h \in H_{f}} A_{i}$. Hence $\left|\mathbf{F}_{\mathscr{E}_{F D l} \downarrow \mathbf{Q}}(X, f)\right|=\sum_{i=1}^{k} c_{i}^{m}$, where $m=$ $\left|H_{f}\right|=\prod_{i=1}^{k} c_{i}^{n_{i}}$. By Corollary 7.5 we get

$$
\begin{aligned}
\left|\mathbf{F}_{\mathscr{E}}(X)\right| & =\prod_{f \in Q^{X}}\left|\mathbf{F}_{\mathscr{E}_{F D} \downarrow}(X, f)\right|=\prod_{f \in Q^{X}} \sum_{i=1}^{k} c_{i}^{m} \\
& =\prod_{\sum_{i=1}^{k} n_{i}=n}\left(\sum_{i=1}^{k} c_{i}^{m}\right)^{\left(n_{1} \cdots n_{k}\right)}
\end{aligned}
$$

(see Demetrovics, Hannak and Ronyai [1982]). 
In the next theorem we find a lower bound for the cardinality of the free indecoposables. Again, let $X$ be a finite set, $\mathscr{V}=H S P(\mathbf{C})$ a finitely generated variety with $F H A P, \mathbf{Q} \in \mathscr{V}_{F S}$ and $f: X \rightarrow Q$ such that $[f(X)]=\mathbf{Q}$. If $\mathbf{B}=$ $\mathbf{F}_{\mathscr{V}_{F D I} \downarrow \mathbf{Q}}(X, f), \bar{f}: \mathbf{B} \rightarrow \mathbf{Q}$, then for $q \in Q, \mathbf{B}_{q}$ denotes, as before, the $d(\mathscr{V})_{q}$-algebra of type $\tau_{q}$ with domain $\bar{f}^{-1}(\{q\})$.

THEOREM 7.7. Let $\mathscr{V}, \mathbf{C}, \mathbf{B}, X, \mathbf{Q}, f$ be as in the previous paragraph.

For $q \in Q$ let $X_{q}=f^{-1}(\{q\})$. Then $\mathbf{F}_{d(\mathscr{V})_{q}}\left(X_{q}\right) \in I S\left(\mathbf{B}_{q}\right)$ and hence

$$
|B| \geqslant \sum_{q \in Q}\left|F_{d(\mathscr{V})_{q}}\left(\left|X_{q}\right|\right)\right| \text {. }
$$

Proof. Let $q \in Q, \mathscr{W}=d(\mathscr{V})_{q}$, and $X_{q}=\left\{x_{1}, \ldots, x_{t}\right\}$. Let $p_{1}, p_{2}$ be $\mathscr{W}$-terms such that $p_{1}\left(x_{1}, \ldots, x_{t}\right) \neq p_{2}\left(x_{1}, \ldots, x_{t}\right)$ in $\mathbf{F}_{\mathscr{W}}\left(X_{q}\right)$. By the definition of $d(\mathscr{V})_{q}$ there exists an object $(\mathbf{A}, g) \in \mathscr{V}_{F D I} \downarrow \mathbf{Q}$ with $a_{1}, \ldots, a_{t} \in A_{q}$ such that

$$
p_{1}\left(a_{1} \cdots a_{t}\right) \neq p_{2}\left(a_{1} \cdots a_{t}\right) \text { in } \mathbf{A}_{q} \text {. }
$$

Now define a map $h: X \rightarrow A$ satisfying $h\left(x_{j}\right)=a_{j}, j=1, \ldots, t, h(x) \in g^{-1}(f(x))$ for $x \in X \backslash X_{q}$. Then $h:(X, f) \rightarrow(A, g)$, and $h$ can thus be extended to a map $\bar{h}$ : $(\mathbf{B}, \bar{f}) \rightarrow(\mathbf{A}, g)$, with $\bar{h} \circ i=h$. Since $\bar{h}\left(p_{j}\left(i\left(x_{1}\right), \ldots, i\left(x_{t}\right)\right)\right)=p_{j}\left(a_{1} \cdots a_{t}\right), j=$ 1,2 , it follows that $\left.p_{1}\left(i\left(x_{1}\right), \ldots, i\left(x_{t}\right)\right) \neq p_{2}\left(x_{1}\right), \ldots, i\left(x_{t}\right)\right)$ in $\mathbf{B}$. Hence the map $x_{j} \mapsto i\left(x_{j}\right), j=1, \ldots, t$ extends to an embedding of $\mathbf{F}_{\mathscr{W}}\left(X_{q}\right)$ into $\mathbf{B}_{q}$.

In many of the examples the lower bound provided by Theorem 7.7 is actually exact. By way of illustration we will discuss the case of Stone algebras (Example 1.1) in detail. In the remarks following Lemma 6.4 we observed that the only subdirectly irreducible Stone algebras are the 2-element Boolean algebra $\mathbf{S}_{2}$ with universe, say, $\{0,1\}$, and the 3-element chain $\mathbf{S}_{3}=\langle\{0, a, 1\}, \wedge, \vee, *, 1\rangle$, with $0<a<1$. Thus $\mathscr{V}=\operatorname{HSP}\left(\mathbf{S}_{3}\right)$ and $\mathscr{V}_{F S}=\left\{\mathbf{S}_{2}\right\}$. We further showed there that $d(\mathscr{V})_{0}$ is trivial and $d(\mathscr{V})_{1}$ is the variety $\mathscr{D}_{1}$ of distributive lattices with unit. Since $\mathbf{S}_{2}$ is primal,

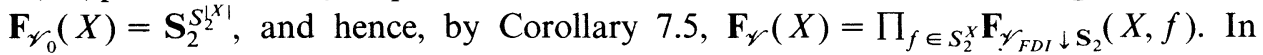
order to determine the free indecomposables, let us fix $f: X \rightarrow S_{2}$ for the remainder of this proof, and write $X_{0}=f^{-1}(\{0\}), X_{1}=f^{-1}(\{1\})$. By Theorem 7.7

$$
\left|F_{\mathscr{V}_{F D I} \downarrow \mathbf{S}_{2}}(X, f)\right| \geqslant\left|F_{d(\mathscr{V})_{0}}\left(\left|X_{0}\right|\right)\right|+\left|F_{d(\mathscr{V})_{1}}\left(\left|X_{1}\right|\right)\right|=1+\left|F_{\mathscr{D}_{1}}\left(\left|X_{1}\right|\right)\right| .
$$

We claim that the inequality can be shaprened to an equality. Without loss of generality we may assume $X=\left\{x_{1}, \ldots, x_{n}\right\}$ with $\left\{x_{1}, \ldots, x_{k}\right\}=X_{0}$, $\left\{x_{k+1}, \ldots, x_{n}\right\}=X_{1}, 0 \leqslant k \leqslant n$. A typical member of $F_{\mathscr{V}_{F D I} \downarrow \mathbf{s}_{2}}(X, f)$ can be written $\pi_{f}\left(t\left(x_{1}, \ldots, x_{n}\right)\right)$, where $t\left(x_{1}, \ldots, x_{n}\right) \in F_{\mathscr{V}}(X)$ and $\pi_{f}$ is the projection $F_{\mathscr{V}}(X)$ $\rightarrow F_{\mathscr{V}_{F D I} \downarrow \mathbf{S}_{2}}(X, f)$. If $i:(X, f) \rightarrow F_{\mathscr{V}_{F D I} \downarrow \mathbf{S}_{2}}(X, f)$ denotes, as usual, the canonical embedding, then $\pi_{f}\left(t\left(x_{1}, \ldots, x_{n}\right)\right)=t\left(i\left(x_{1}\right), \ldots, i\left(x_{n}\right)\right)$, evaluated in $F_{\mathscr{V}_{F D I} \downarrow \mathbf{S}_{2}}(X, f)$. We will show that either $t\left(i\left(x_{1}\right), \ldots, i\left(x_{n}\right)\right)=1^{*}$ or there is a $\mathscr{D}_{1}$-term $r$ of arity $\left|X_{1}\right|$ such that $t\left(i\left(x_{1}\right), \ldots, i\left(x_{n}\right)\right)=r\left(i\left(x_{k+1}\right), \ldots, i\left(x_{n}\right)\right)$. This will prove that $\left|F_{\mathscr{V}_{F D} \downarrow \mathbf{S}_{2}}(X, f)\right| \leqslant 1+\left|F_{\mathscr{D}_{1}}\left(\left|x_{1}\right|\right)\right|$.

By Theorem 7.6, $p: \mathbf{F}_{\mathscr{V}_{F D I} \downarrow \mathbf{S}_{2}}(X, f) \rightarrow \prod_{s \in S} \mathbf{C}_{s}$ is a subdirect embedding, where $\mathbf{C}_{s} \in\left\{\mathbf{S}_{2}, \mathbf{S}_{3}\right\}$, and $p_{s}:\left(\mathbf{F}_{\mathscr{V}_{F D I} \downarrow \mathbf{S}_{2}}(X), \bar{f}\right) \rightarrow\left(\mathbf{C}_{s}, f_{s}\right)$, and we may think of $F_{\mathscr{V}_{F D I} \downarrow \mathbf{S}_{2}}(X, f)$ as a subalgebra of $\Pi_{s \in S} \mathbf{C}_{s}$. If $\bar{f}(t)=0$, then $f_{s}\left(p_{s}\left(t\left(i\left(x_{1}\right), \ldots, i\left(x_{n}\right)\right)\right)\right)=0$ for all $s \in S$, hence $t\left(i\left(x_{1}\right), \ldots, i\left(x_{n}\right)\right)=0=1^{*}$. In 
particular, $i\left(x_{j}\right)=0,1 \leqslant j \leqslant k$. If $\bar{f}(t)=1$, then $f_{s}\left(p_{s}\left(t\left(i\left(x_{1}\right), \ldots, i\left(x_{n}\right)\right)\right)\right)=1$, hence $p_{s}\left(t\left(i\left(x_{1}\right), \ldots, i\left(x_{n}\right)\right)\right) \in\{a, 1\}$, for all $s \in S$. In this case, there is a $\mathscr{D}_{1}$-term $r\left(x_{k+1}, \ldots, x_{n}\right)$ such that $t\left(i\left(x_{1}\right), \ldots, i\left(x_{n}\right)\right)=r\left(i\left(x_{n+1}\right), \ldots, i\left(x_{n}\right)\right)$ in $F_{\mathscr{V}_{F D I} \downarrow \mathbf{S}_{2}}(X, f)$. We show this by induction on the complexity of $t$. If $t=x_{j}$, then since $f(t)=1, k+1 \leqslant j \leqslant n$, and $r=x_{j}$, will do. The induction steps involving $\vee, \wedge$, and 1 present no problems. Finally, if $t=u^{*}$, then since $\vec{f}(t)=1, \bar{f}(u)=0$, and thus $u\left(i\left(x_{1}\right), \ldots, i\left(x_{n}\right)\right)=0$, whence $t\left(i\left(x_{1}\right), \ldots, i\left(x_{n}\right)\right)=1$. In this case we can use the $\mathscr{D}_{1}$-term $r=1$. We have thus shown that $\left|F_{\mathscr{V}_{F D I} \downarrow \mathbf{S}_{2}}(X, f)\right|=1+\left|F_{\mathscr{D}_{1}}\left(\left|X_{1}\right|\right)\right|$. Finally, for $0 \leqslant j \leqslant n,\left|\left\{f \in 2^{X}:\left|f^{-1}(\{1\})\right|=j\right\}\right|=\left(\begin{array}{c}n \\ j\end{array}\right)$, hence

$$
\left|F_{\mathscr{V}}(X)\right|=\prod_{j=0}^{n}\left(1+\left|F_{\mathscr{D}_{1}}(j)\right|\right)^{\left(\begin{array}{c}
n \\
j
\end{array}\right)} \text {. }
$$

The same method of proof applies more generally.

Fact 7.8. Let $\mathscr{V}, \mathbf{C}, \mathbf{Q}, X$, and $f: X \rightarrow Q$ be as in the paragraph before 7.7. Set $X_{q}=f^{-1}(\{q\}), q \in Q$. Then $\left|F_{\mathscr{V}_{F D I} \downarrow Q}(X, f)\right|=\sum_{q \in Q}\left|F_{d(\mathscr{V})_{q}}\left(X_{q}\right)\right|$ if the following condition is satisfied.

(7.9) For every $n$-ary $\mathscr{V}$-term $t\left(x_{1}, \ldots, x_{n}\right)$ there is a $d(\mathscr{V})_{q}$-term (of type $\tau_{q}$ ) $t_{q}\left(x_{i_{1}}, \ldots, x_{i_{1}}\right)$ where $q=t\left(f\left(x_{1}\right), \ldots, f\left(x_{n}\right)\right), X_{q}=\left\{x_{i_{1}}, \ldots, x_{i_{1}}\right\}$, such that

(7.10) for all $j:(X, f) \rightarrow\left(C_{s}, f_{s}\right), s \in S$,

$$
t\left(j\left(x_{1}\right), \ldots, j\left(x_{n}\right)\right)=t_{q}\left(j\left(x_{i_{1}}\right), \ldots, j\left(x_{i_{i}}\right)\right) \text { in } \mathbf{C}_{s} .
$$

Note that in this case $t$ does not depend on the variables in $X \backslash X_{q}$. If condition 7.9 is met, then the assignment $t \mapsto\left(X_{q}, t_{q}\right)$ is $1-1$, and hence $\left|F_{\mathscr{V}_{F D I} \downarrow \mathbf{Q}}(X, f)\right| \leqslant$ $\sum_{q \in Q}\left|F_{d(\mathscr{V})_{q}}\left(X_{q}\right)\right|$. Recall that the reverse inequality always holds by virtue of Theorem 7.7.

In fact, we need to verify (7.9) only for the fundamental operations:

THEOREM 7.11. Suppose for all finite $X=\left\{x_{1}, \ldots, x_{n}\right\}$ and all $f: X \rightarrow Q$ such that $[f(X)]=\mathbf{Q}$, and for every fundamental operation $c\left(x_{1}, \ldots, x_{n}\right)$ there exists a $d(\mathscr{V})_{q^{-}}$ term $c_{q}\left(x_{i_{1}}, \ldots, x_{i_{l}}\right)$ where $q=f\left(x_{1}, \ldots, x_{n}\right),\left\{x_{i_{1}}, \ldots, x_{i_{l}}\right\}=f^{-1}(\{q\})$, such that (7.10) holds, i.e.,

(7.12) for all $j:(X, f) \rightarrow\left(C_{s}, f_{s}\right), s \in S$,

$$
c\left(j\left(x_{1}\right), \ldots, j\left(x_{n}\right)\right)=c_{q}\left(j\left(x_{i_{1}}\right), \ldots, j\left(x_{i_{1}}\right)\right) \text { in } C_{s} .
$$

Then

$$
\left|F_{\mathscr{V}_{F D I} \downarrow \mathbf{Q}}(X, f)\right|=\sum_{q \in Q}\left|F_{d(\mathscr{V})_{q}}\left(X_{q}\right)\right| .
$$

Proof. We show by induction on the complexity of the terms that (7.10) is met. If $t\left(x_{1}, \ldots, x_{n}\right)=x_{i}$, for some $i, 1 \leqslant i \leqslant n$, we use $t_{q}\left(x_{i}\right)=x_{i}$. Now suppose that for every $f: X \rightarrow Q, X$ finite, $[f(X)]=\mathbf{Q}$, and all terms in $X$ of complexity $<m(7.10)$ is satisfied. Let $X$ be finite, say, $X=\left\{x_{1}, \ldots, x_{n}\right\}, f: X \rightarrow Q,[f(X)]=\mathbf{Q}$, and let $t\left(x_{1}, \ldots, x_{n}\right)$ be a $\mathscr{V}$-term of complexity $m$. Then

$$
t\left(x_{1}, \ldots, x_{n}\right)=c\left(t_{1}\left(x_{1}, \ldots, x_{n}\right), \ldots, t_{k}\left(x_{1}, \ldots, x_{n}\right)\right),
$$

where $c\left(x_{1}, \ldots, x_{k}\right)$ is a fundamental operation, and $t_{i}\left(x_{1}, \ldots, x_{n}\right)$ are terms of complexity $<m, i=1, \ldots, k$. Let

$$
q=t\left(f\left(x_{1}\right), \ldots, f\left(x_{n}\right)\right), \quad \text { and } \quad q_{i}=t_{i}\left(f\left(x_{1}\right), \ldots, f\left(x_{n}\right)\right), \quad i=1, \ldots, k .
$$


Now let $f^{\prime}: X^{\prime} \rightarrow Q$, with $X^{\prime}$ finite, $\left\{x_{1}, \ldots, x_{k}\right\} \subseteq X^{\prime},\left[f^{\prime}\left(X^{\prime}\right)\right]=\mathbf{Q}$, such that $f^{\prime}\left(x_{i}\right)=q_{i}, i=1, \ldots, k$. By hypothesis there exists a $d(\mathscr{V})_{q}$-term $c_{q}\left(x_{i_{1}}, \ldots, x_{i_{1}}\right)$, where $\left\{x_{i_{1}}, \ldots, x_{i_{1}}\right\}=\left(f^{\prime}\right)^{-1}(\{q\})$, such that (7.12) holds for $\left(X, f^{\prime}\right), c$, and $c_{q}$. In particular,

$$
\begin{aligned}
& c\left(t_{1}\left(j\left(x_{1}\right), \ldots, j\left(x_{n}\right)\right), \ldots, t_{k}\left(j\left(x_{1}\right), \ldots, j\left(x_{n}\right)\right)\right) \\
& \quad=c_{q}\left(t_{i_{1}}\left(j\left(x_{1}\right), \ldots, j\left(x_{n}\right)\right), \ldots, t_{i_{l}}\left(j\left(x_{1}\right), \ldots, j\left(x_{n}\right)\right)\right)
\end{aligned}
$$

for all $j:\left(X, f^{\prime}\right) \rightarrow\left(C_{s}, f_{s}\right)$. By the induction hypothesis, the $t_{i_{j}}\left(x_{1}, \ldots, x_{n}\right)$ can in turn be replaced by terms $\left(t_{i_{j}}\right)_{q}\left(x_{j_{1}}, \ldots, x_{j_{m}}\right)$, where $X_{q}=\left\{x_{j_{1}}, \ldots, x_{j_{m}}\right\}$. Thus the $d(\mathscr{V})_{q}$-term to be assigned to $t\left(x_{1}, \ldots, x_{n}\right)$ is

$$
c_{q}\left(\left(t_{i_{1}}\right)_{q}\left(x_{j_{1}}, \ldots, x_{j_{m}}\right), \ldots,\left(t_{i_{1}}\right)_{q}\left(x_{j_{1}}, \ldots, x_{j_{m}}\right)\right) \text {. }
$$

REMARK 7.13. The tests (7.10) and (7.12) can in many cases be simplified considerably. Suppose $\mathbf{C}$ is directly indecomposable, $f^{*}: \mathbf{C} \rightarrow \mathbf{Q}$ an onto homomorphism. If for every $s \in S$ there is a $\mathbf{C}_{s}^{\prime} \in S(\mathbf{C})$ and $h_{s}: \mathbf{C}_{s}^{\prime} \rightarrow \mathbf{C}_{2}$, such that the pair $\left(\mathbf{C}_{s}^{\prime}, f_{s} \circ h_{s}\right)$ can be embedded in $\left(\mathbf{C}, f^{*}\right)$, i.e., there exists an embedding $j_{s}$ : $\left(\mathbf{C}_{s}^{\prime}, f_{s} \circ h_{s}\right) \rightarrow\left(\mathbf{C}, f^{*}\right)$, then clearly it will suffice to verify (7.10), resp. (7.12) only for all $j:(X, f) \rightarrow\left(C, f^{*}\right)$.

These observations make it possible to determine with little effort the free spectra of the varieties listed in Table 1 . All of them contain only one simple algebra-the 2-element Boolean algebra, the 2-element Boolean ring, or the 2-element generalized Boolean algebra - and Remark 7.13 applies in all these cases. In addition to the examples so far, Table 1 also includes the variety of Heyting algebras (e.g., Balbes and Dwinger [1974]) generated by a 3-element chain; the analysis is similar to that for Brouwerian algebras.

In a similar way we can determine the free spectrum of the variety $\mathscr{V}=H S P(\mathbf{A})$ of Example 6.7. The only simple algebra here is the primal algebra $\mathbf{Q}$. Let $X$ be a finite set, $f: X \rightarrow Q$ such that $[f(X)]=\mathbf{Q}$ and let $f^{*}: \mathbf{A} \rightarrow \mathbf{Q}$. If. $\mathbf{A}^{\prime} \in S(\mathbf{A})$, then $\mathbf{A}^{\prime}$ is directly indecomposable, in fact, there is a unique homomorphism $f^{\prime}: \mathbf{A}^{\prime} \rightarrow \mathbf{Q}$, and the $\left(\mathbf{A}^{\prime}, f^{\prime}\right)$ can in turn be embedded in $\left(A, f^{*}\right)$. Thus we need to test (7.12) only for morphisms $j:(X, f) \rightarrow\left(A, f^{*}\right)$. We apply Theorem 7.11. Let $X$ be finite, $X \supseteq\left\{x_{1}, x_{2}\right\}, f: X \rightarrow Q$ arbitrary. Then $[f(X)]=\mathbf{Q}$. If $f\left(x_{1}\right)=f\left(x_{2}\right)=q$ then for all $j:(X, f) \rightarrow\left(A, f^{*}\right), j\left(x_{1}\right)+j\left(x_{2}\right)=j\left(x_{1}\right) \oplus j\left(x_{2}\right)$; hence $\oplus$ is the sought for $d(\mathscr{V})_{q}$-term. If $f\left(x_{1}\right)<f\left(x_{2}\right)=q$, then $j\left(x_{1}\right)+j\left(x_{2}\right)=j\left(x_{2}\right)$; hence $x_{2}$ is the required $d(\mathscr{V})_{q}$-term. The operations $\circ$ and $*$ are treated similarly. Thus

$$
\left|F_{\mathscr{V}_{F D I} \downarrow \mathbf{Q}}(X, f)\right|=\sum_{i=0}^{m-1}\left|F_{H S P\left(\mathbf{A}_{i}\right)}\left(X_{i}\right)\right|,
$$

and

$$
\left|F_{\mathscr{V}}(n)\right|=\prod_{n_{0}+\cdots+n_{m-1}=n}\left(\sum_{i=0}^{m-1}\left|F_{H S P\left(\mathbf{A}_{i}\right)}\left(n_{i}\right)\right|\right)^{\left(n_{0}, \ldots \ldots n_{m-1}\right)} .
$$

In the variety of complemented semigroups (Example 6.9), there are two simple algebras $\mathbf{Q}_{1}$ and $\mathbf{Q}_{2}$. The generator of the variety, $\mathbf{B}$, is directly indecomposable, and $B / \alpha \cong \mathbf{Q}_{1}$. Let $h: \mathbf{B} \rightarrow \mathbf{Q}_{1}$, be a surjective homomorphism. Given a finite set $X$, 
and a map $f: X \rightarrow Q_{1}$, such that $[f(X)]=\mathbf{Q}_{1}$ (i.e., $X_{0} \neq \varnothing$ ), we see as before that $\left|F_{\mathscr{V}_{F D I} \downarrow Q_{1}}(X, f)=\right| F_{d(\mathscr{V})_{0}}\left(X_{0}\right)|+| F_{d(\mathscr{V})_{1}}\left(X_{1}\right) \mid$; since $d(\mathscr{V})_{0}$ is trivial and $d(\mathscr{V})_{1}$ is the variety of Boolean rings, we get $\left|F_{\mathscr{V}_{F D I} \downarrow \mathbf{Q}_{1}}(X, f)\right|=1+2^{2^{\left|X_{1}\right|}-1}$. For a map $f$ : $X \rightarrow Q_{2}$ such that $[f(X)]=\mathbf{Q}_{2}$ (i.e., $X_{0} \neq \varnothing$ ) we find similarly $\left|F_{\mathscr{V}_{F D I} \downarrow \mathbf{Q}_{2}}(X, f)\right|=$ $1+1=2$. Let $\mathscr{V}_{0}=\operatorname{HSP}\left(\left\{\mathbf{Q}_{1}, \mathbf{Q}_{2}\right\}\right)$, then $\mathbf{F}_{\mathscr{V}_{0}}(n) \cong \mathbf{Q}_{1}^{2^{n}-1} \times \mathbf{Q}_{2}^{2^{n}-1}$. Hence

$$
\left|F_{\mathscr{V}}(n)\right|=\left[\prod_{n_{1}=0}^{n-1}\left(1+2^{2^{n_{1}}-1}\right)^{\left(\begin{array}{c}
n \\
n_{1}
\end{array}\right)}\right] \cdot 2^{2^{n}-1} .
$$

Thus far we have discussed the case where $\left|\mathbf{F}_{\mathscr{V}}(n)\right|$ is minimal relative to $\mathscr{V}_{0}$ and $d(\mathscr{V})_{q}, q \in Q, \mathbf{Q} \in \mathscr{V}_{F S}$. We will now consider a condition on $\mathscr{V}$ which will provide a larger lower bound for $\left|F_{\mathscr{V}}(n)\right|$.

THEOREM 7.15. Let $\mathbf{C}$ be a finite algebra, and suppose $\mathscr{V}=H \operatorname{HP}(\mathbf{C})$ has FHAP. Let $\mathbf{Q} \in \mathscr{V}_{F S}, X$ a finite set and $f: X \rightarrow Q$ such that $[f(X)]=\mathbf{Q}, q \in Q$. Suppose that for all $q^{\prime} \in Q$ there are unary terms $u_{q^{\prime} q}$ such that for all $\mathbf{A} \in H S(\mathbf{C})_{S I} \downarrow \mathbf{Q}$, $u_{q^{\prime} q}\left(A_{q^{\prime}}\right)=A_{q}$. Then $\left|F_{\mathscr{V}_{F D I} \downarrow \mathbf{Q}}(X, f)_{q}\right| \geqslant\left|F_{d(\mathscr{V})_{q}}(X)\right|$.

Proof. By Theorem 6.3(iii), $d(\mathscr{V})_{q}=H S P\left\{\mathbf{A}_{q}: \mathbf{A} \in H S(\mathbf{C})_{S I} \downarrow \mathbf{Q}\right\}$, hence $\mathbf{F}_{d(\mathscr{V})_{q}}(X) \in I S P\left\{\mathbf{A}_{q}: \mathbf{A} \in H S(\mathbf{C})_{S I} \downarrow \mathbf{Q}\right\}$. Let $\left(\mathbf{C}_{s}, f_{s}\right) \in H S(\mathbf{C})_{S I} \downarrow \mathbf{Q}, s=1, \ldots, m$, and $k: \mathbf{F}_{d(\mathscr{V})_{q}}(X) \rightarrow \prod_{s=1}^{m}\left(\mathbf{C}_{s}\right)_{q}$ be an embedding. We claim there is a homomorphism

$$
h: \mathbf{F}_{\mathscr{V}_{F D I} \downarrow \mathbf{Q}}(X, f) \rightarrow \prod_{s=1}^{m} \mathbf{C}_{s}
$$

such that

$$
h\left(\left(F_{\mathscr{V}_{F D I} \downarrow \mathbf{Q}}(X, f)\right)_{q}\right) \supseteq k\left(F_{d\left(\mathscr{V}_{q}\right)}(X)\right) .
$$

Write $X=\left\{x^{1}, \ldots, x^{n}\right\}$. Choose $y_{s}^{j} \in\left(C_{s}\right)_{f\left(x^{j}\right)}$ in such a way that $u_{f\left(x^{j}\right) q}\left(y_{s}^{j}\right)=$ $\left(k\left(x^{j}\right)\right)_{s}$. This is possible by hypothesis. Let $h_{s}: X \rightarrow C_{s}$ be defined by $h_{s}\left(x^{j}\right)=y_{s}^{j}$, $j=1, \ldots, n$. By the choice of $y_{s}^{j}$ we actually have $h_{s}:(X, f) \rightarrow\left(C_{s}, f_{s}\right)$. Let $\bar{h}_{s}$ : $\left(\mathbf{F}_{\mathscr{V}_{F D I} \downarrow \mathbf{Q}}(X, f), \bar{f}\right) \rightarrow\left(\mathbf{C}_{s}, f_{s}\right)$ be the extension, and let $h=\prod_{s=1}^{m} \bar{h}_{s}: \mathbf{F}_{\mathscr{V}_{F D I} \downarrow \mathbf{Q}}(X, f)$ $\rightarrow \prod_{s=1}^{m} \mathbf{C}_{s}$. Then $h \circ i=\prod_{s=1}^{n} h_{s}$, and thus $h \circ i\left(x^{j}\right)=y^{j}, j=1, \ldots, n$. In order to check that $h$ satisfies $(*)$, let $z=w\left(x^{1}, \ldots, x^{n}\right) \in F_{d(\mathscr{V})_{q}}(X)$ be arbitrary. Then

$$
\begin{aligned}
k(z) & =w\left(k\left(x^{1}\right), \ldots, k\left(x^{n}\right)\right) \\
& =\left(w\left(k\left(x^{1}\right)_{s}, \ldots, k\left(x^{n}\right)_{s}\right)\right)_{s=1}^{m} \\
& =\left(w\left(u_{f\left(x^{1}\right) q}\left(y_{s}^{1}\right), \ldots, u_{f\left(x^{n}\right) q}\left(y_{s}^{n}\right)\right)\right)_{s=1}^{m} \\
& =w\left(u_{f\left(x^{1}\right) q}\left(y^{1}\right), \ldots, u_{f\left(x^{n}\right) q}\left(y^{n}\right)\right) \\
& =w\left(u_{f\left(x^{1}\right) q}\left(h \circ i\left(x^{1}\right)\right), \ldots, u_{f\left(x^{n}\right) q}\left(h \circ i\left(x^{n}\right)\right)\right) \\
& =h\left(w\left(u_{f\left(x^{1}\right) q}\left(i\left(x^{1}\right), \ldots, u_{f\left(x^{n}\right) q}\left(i\left(x^{n}\right)\right)\right)\right)\right) .
\end{aligned}
$$


Furthermore,

$$
f\left(w\left(u_{f\left(x^{1}\right) q}\left(i\left(x^{1}\right)\right), \ldots, u_{f\left(x^{n}\right) q}\left(i\left(x^{n}\right)\right)\right)\right)=w(q, \ldots, q)=q .
$$

That completes the proof of $(*)$. The conclusion of the theorem is now immediate.

In the next theorem a condition is given which guarantees that the lower bound of Theorem 7.15 is also an upper bound:

THEOREM 7.16. Under the hypotheses of Theorem 7.15 now suppose that for all $q$, $q^{\prime} \in Q$ there are unary terms $v_{q q^{\prime}}$ such that for all $\mathbf{A} \in H S(\mathbf{C})_{S I} \downarrow \mathbf{Q}, v_{q q^{\prime}}\left(A_{q}\right)=A_{q^{\prime}}$. Then $\mathbf{F}_{\mathscr{V}_{F D I} \downarrow \mathbf{Q}}(X, f)_{q} \in S H\left(\mathbf{F}_{d(\mathscr{V})_{q}}(X)\right)$, and hence

$$
\left|F_{\mathscr{V}_{F D I} \downarrow \mathbf{Q}}(X, f)\right| \leqslant \sum_{q \in Q}\left|F_{d(\mathscr{r})_{q}}(X)\right| \text {. }
$$

Proof. By Theorem 7.6, we may assume $\mathbf{F}_{\mathscr{V}_{F D I} \downarrow \mathbf{Q}}(X, f)=\mathbf{B}$ is a subalgebra of the algebra $\Pi_{s \in S} \mathbf{C}_{s}$, with $\mathbf{C}_{s} \in H S(\mathbf{B})_{S I} \downarrow Q$, and homomorphisms $p_{s}(\mathbf{B}, \bar{f}) \rightarrow\left(\mathbf{C}_{s}, f_{s}\right)$. Let $X=\left\{x^{1}, \ldots, x^{n}\right\}$; then $\tilde{f}\left(i\left(x^{j}\right)\right)=f_{s}\left(i\left(x^{j}\right)_{s}\right), s \in S, j=1, \ldots, n$. Set $q^{j}=$ $\bar{f}\left(i\left(x^{j}\right)\right), j=1, \ldots, n$. Choose for $s \in S, j=1, \ldots, n$, an element $y_{s}^{j} \in\left(C_{s}\right)_{q}$ such that $v_{q q^{j}}\left(y_{s}^{j}\right)=i\left(x^{j}\right)_{s}$, and let $y^{j}=\left(y_{s}^{j}\right)_{s \in S}, j=1, \ldots, n$. Let $\mathbf{D}$ be the $d(\mathscr{V})_{q^{-}}$ algebra generated by $\left\{y^{1}, \ldots, y^{n}\right\}$. Note that $\mathbf{D} \in H\left(\mathbf{F}_{d(\mathscr{V})_{q}}(X)\right)$ and $D \subseteq$ $\prod_{s \in S}\left(C_{s}\right)_{q}$. Let $z=w\left(i\left(x^{1}\right), \ldots, i\left(x^{n}\right)\right) \in B_{q}$. Then $z=w\left(v_{q q^{1}}\left(y^{1}\right), \ldots, v_{q q^{n}}\left(y^{n}\right)\right)$. Furthermore, for $s \in S$,

$$
\begin{aligned}
q & =f_{s}\left(z_{s}\right)=f_{s}\left(w\left(v_{q q^{\prime}}\left(y_{s}^{1}\right), \ldots, v_{q q^{n}}\left(y_{s}^{n}\right)\right)\right) \\
& =w\left(v_{q q^{1}}(q), \ldots, v_{q q^{n}}(q)\right) .
\end{aligned}
$$

Thus $z$ belongs to the $d(\mathscr{V})_{q}$-subalgebra generated by $y^{1}, \ldots, y^{n}$, and hence $B_{q} \subseteq D$. That completes the proof of the theorem.

The condition on the existence of the unary terms $v_{q q^{\prime}}$ cannot be omitted. For example, if in Example 1.3 we let $A=\{a, b, c, d, e\}, \alpha=a b \mid c d e$ and $A / \alpha=\{1,2\}$, $\mathbf{Q}=\mathbf{A} / \alpha$, then $c_{1}=2, c_{2}=3$, and hence $\left|F_{d(\mathscr{V})_{1}}(n)\right|=2^{2^{n}},\left|F_{d(\mathscr{V})_{2}}(n)\right|=3^{3^{n}}$. Let $X=\{x\}$, and $f:\{x\} \rightarrow\{1,2\}$ be given by $f(x)=2$. Let $\mathbf{B}=\mathbf{F}_{\mathscr{V}_{F D I} \downarrow \mathbf{Q}}(X, f)$, then we get $\left|B_{1}\right|=2^{3}>2^{2}$; hence $\mathbf{B}_{1}$ is not a subalgebra of a 1-generated $d(\mathscr{V})_{1}$-algebra.

A more general version of Theorem 7.16 can be proven. Its hypotheses are the same as those of 7.16, except we suppose now that for each $q^{\prime} \in Q$ there is an $n_{q^{\prime}}$-ary term $t_{q q^{\prime}}\left(x_{1}, \ldots, x_{n_{q^{\prime}}}\right)$ such that $t_{q q^{\prime}}\left(A^{n_{q^{\prime}}}\right)=A_{q^{\prime}}$ for all $A \in H S(\mathbf{C})_{S I} \downarrow \mathbf{Q}$. Then a similar argument shows $\mathbf{B}_{q}$ is a subalgebra of an $\left(\sum_{j=1}^{n} n_{f\left(x^{j}\right)}\right)$-generated $d(\mathscr{V})_{q}$-algebra. Hence $\left|B_{q}\right| \leqslant\left|F_{d(\mathscr{V})_{q}}\left(\sum_{j=1}^{n} n_{f\left(x^{j}\right)}\right)\right|$.

Putting Theorems 7.15 and 7.16 together, we get

COROLlaRY 7.17. If for all $q, q^{\prime} \in Q$ there are unary terms $u_{q q^{\prime}}, v_{q^{\prime} q}$ such that for all $\mathbf{A} \in H S(\mathbf{C})_{S I} \downarrow \mathbf{Q} u_{q q^{\prime}}: A_{q} \rightarrow A_{q^{\prime}}$ and $v_{q^{\prime} q}: A_{q^{\prime}} \rightarrow A_{q}$ are both onto, then $\mathbf{B}_{q} \cong$ $\mathbf{F}_{d(\mathscr{V})_{q}}(X)$, for $q \in Q$, and hence $|B|=\sum_{q \in Q}\left|F_{d(\mathscr{V})_{q}}(X)\right|$.

EXAMPLE 7.18. As an example consider the variety $\mathscr{I}_{3}$ of interior algebras (cf. Example 1.4; Table 2). Here $C=\{0, a, b, 1\}$, with $0<a, b<1, b=a^{\prime}$, and $\square a=\square 0=0, \square b=b, \square 1=1$. The unique coatom $\alpha$ of $\operatorname{Co}(\mathbf{C})$ has partition $0 a \mid b 1$. Let $\mathbf{Q}=\mathbf{C} / \alpha$ be the interior algebra on $\{0,1\}$, then $C_{0}=\{0, a\}, C_{1}=\{b, 1\}$. The 
class $H S(\mathbf{C})_{S I} \downarrow \mathbf{Q}$ consists of $\mathbf{C}$ and $\mathbf{Q}$, and hence we can take $u_{10}$ and $u_{01}$ to be complementation. As observed in $\S 6 d\left(\mathscr{I}_{3}\right)_{1}$ is the variety $\mathscr{I}_{2}^{-}$generated by the 2-element generalized interior algebra with $1, \mathbf{I}_{2}^{-}$, while $d\left(\mathscr{I}_{3}\right)_{0}$ is the variety $\tilde{\mathscr{I}}_{2}^{-}$ generated by the 2-element generalized closure algebra with 0 . Given $f: X \rightarrow Q$ we find, by Corollary 7.17 ,

$$
\begin{aligned}
\left|F_{\left(\mathscr{J}_{3}\right)_{F D} \downarrow \mathbf{Q}}(X, f)\right| & =\left|F_{d\left(\mathscr{J}_{3}\right)_{0}}(X)\right|+\left|F_{d\left(\mathscr{\mathcal { S }}_{3}\right)_{1}}(X)\right| \\
& =2^{2^{|X|}-1}+2^{2^{|X|}-1}=2^{2^{|X|}} .
\end{aligned}
$$

Thus $\left|F_{\mathscr{J}_{3}}(n)\right|=\prod_{f \in Q^{n}} 2^{2^{n}}=\left(2^{2^{n}}\right)^{2^{n}}=2^{2^{2 n}}$.

EXAMPLE 7.19. Another application of the corollary is found in the variety $\mathscr{Z}_{p^{2}}=H S P\left(\mathbf{Z}_{p^{2}}\right)$ (Example 5.5). In $\$ 5$ we verified it has FHAP, with $\mathbf{Z}_{p}$ the only simple in $\mathscr{Z}_{p^{2}}$. In $\S 6$ we verified for $p=2$ that $d\left(\mathscr{Z}_{p^{2}}\right)_{i}, 0 \leqslant i \leqslant p-1$, is the variety $\mathscr{A} b_{p}^{*}$ of Abelian $p$-groups with constants; in a similar way one can show this holds for all prime $p$. Given $i, j \in Z_{p}$, let $u_{i j}(x)=(x+j-1) \bmod (p)$. Then $u_{i j}$ is $1-1$ and onto, and thus the hypotheses of Corollary 7.17 are satisfied with $v_{i j}=u_{i j}^{-1}$. We find for $f: X \rightarrow Z_{p}$

$$
\left|F_{\left(\mathscr{Z}_{\left.p^{2}\right)_{F D I} \downarrow} \mathbf{Z}_{p}\right.}(X, f)\right|=p \cdot\left|F_{\mathscr{A} b_{p}^{*}}(X)\right|=p \cdot p^{|X|+1}=p^{|X|+2} .
$$

Hence

$$
\left|F_{\mathscr{Z}_{p^{2}}}(n)\right|=\left|\prod_{f \in Z_{p}^{n}} F_{\left(\mathscr{Z}_{p^{2}}\right)_{F D I} \downarrow \mathbf{Z}_{p}}(X, f)\right|=\left(p^{n+2}\right)^{p^{n}} .
$$

8. Varieties which have the FHAP hereditarily. In $\$ 6$ we associated with a finitely generated variety $\mathscr{V}$ which has FHAP the family $d(\mathscr{V})$ of derived varieties $\left\langle d(\mathscr{V})_{q} \mid q \in Q, \mathbf{Q} \in \mathscr{V}_{F S}\right\rangle$. If all of those have FHAP themselves, we can repeat the process. We can thus assign to any finitely generated variety a tree, whose nodes are labeled by varieties. More precisely, given any finitely generated variety $\mathscr{V}$, let

$$
d(\mathscr{V})= \begin{cases}\left\langle d(\mathscr{V})_{q} \mid q \in Q, \mathbf{Q} \in \mathscr{V}_{F S}\right\rangle & \text { if } \mathscr{V} \text { has FHAP } \\ \varnothing & \text { otherwise. }\end{cases}
$$

Note that if $\mathscr{V}$ is a trivial variety, then $d(\mathscr{V})=\varnothing$. Let $T(\mathscr{V})$ be the tree with root $\mathscr{V}$, immediate successors $d(\mathscr{V})_{q}, q \in Q, \mathbf{Q} \in \mathscr{V}_{F S}$, and subtrees $T\left(d(\mathscr{V})_{q}\right), q \in Q$, $\mathbf{Q} \in \mathscr{V}_{F S}$. If $\mathscr{V}$ is generated by a finite set $\mathscr{K}$ of finite algebras, each of cardinality at most $k$, then no branch of $T(\mathscr{V})$ can contain more than $k$ nodes. For by 6.3(iii), each variety $d(\mathscr{V})_{q}, q \in Q, \mathbf{Q} \in \mathscr{V}_{F S}$ is generated by algebras $A_{q}$, with $\mathbf{A} \in$ $H S(\mathscr{K})_{S I}$, and $\left|A_{q}\right|<|A|$ since simple algebras are nontrivial. Furthermore, by Theorem 6.3(iii), all $d(\mathscr{V})_{q} \in d(\mathscr{V})$ are finitely generated, and hence, by Theorem 4.6, $d(\mathscr{V})$ and $d\left(d(\mathscr{V})_{q}\right)$ are finite sets, for all $q \in Q, \mathbf{Q} \in \mathscr{V}_{F S}$. Thus $T(\mathscr{V})$ is a tree all of whose nodes have finite degree and the length of whose paths is bounded by $k$ and hence must be finite. 
EXAMPLES. The tree of the variety of Stone algebras (Example 1.1) is as shown in Diagram B.

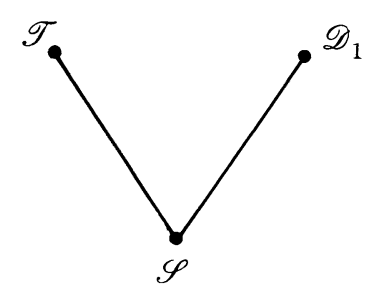

Diagram B

Here $\mathscr{D}_{1}$ is the variety of distributive lattices with $1, \mathscr{T}$ the trivial variety.

The tree of the variety $\mathscr{B}_{k}$ (Example 1.2) is Diagram C.

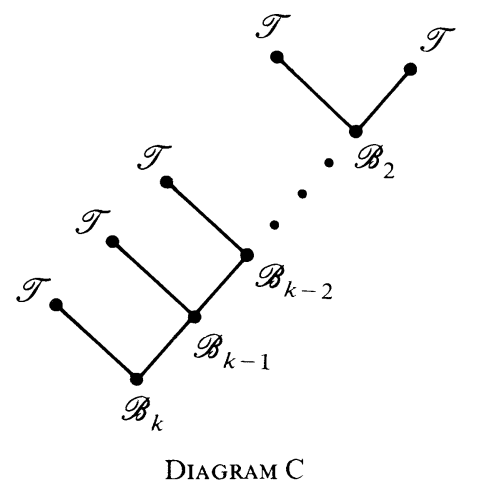

If $\mathscr{E}$ is the variety defined in Example 1.3 , then $T(\mathscr{E})$ is Diagram D.

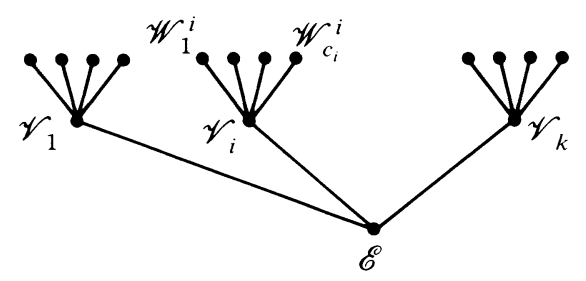

Diagram D

Here $\mathscr{V}_{i}=H S P\left(\mathbf{A}_{i}\right)$, with $\mathbf{A}_{i}$ a $c_{i}$-element primal algebra, $i=1, \ldots, k$, and $\mathscr{W}_{j}^{i}$ is a trivial variety of the appropriate similarity type.

Finally, the tree of the variety $\mathscr{I}_{3}$ of interior algebras (Example 1.4; cf. Example 7.18) is as shown in Diagram E.

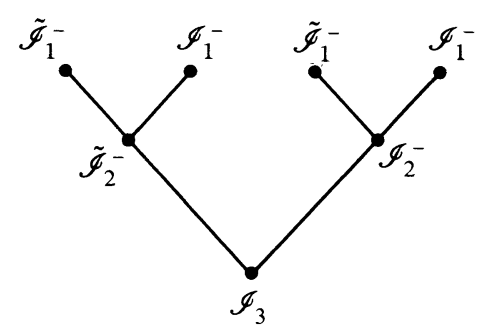

Diagram E 
DEFINITION 8.1. A finitely generated variety $\mathscr{V}$ has FHAP hereditarily if all of the end nodes of its tree $T(\mathscr{V})$ are labeled by a trivial variety.

Thus, except for the variety of Stone algebras, all examples just given have the FHAP hereditarily. Furthermore, all of them are arithmetical-and that is no coincidence.

LEMMA 8.2. Let $\mathscr{V}$ be a finitely generated variety with FHAP. Suppose that every $d(\mathscr{V})_{q}$ is arithmetical. Then so is $\mathscr{V}$.

Proof. For $d(\mathscr{V})_{q} \in d(\mathscr{V})$, let $m_{q}(x, y, z)$ be a Pixley term for $d(\mathscr{V})_{q}$. Let $\mathbf{Q} \in \mathscr{V}_{F S}, \quad X=\left\{x_{1}, x_{2}, x_{3}\right\}, \quad f: \quad X \rightarrow Q$ such that $[f(x)]=\mathbf{Q}$, and $\mathbf{B}_{f}=$ $\mathbf{F}_{\mathscr{V}_{F D I} \downarrow \mathbf{Q}}(X, f)$ the free directly indecomposable of $\mathscr{V} f$-freely generated by $\left\{x_{1}, x_{2}, x_{3}\right\}$. Let

$$
m_{f}\left(x_{1}, x_{2}, x_{3}\right)= \begin{cases}m_{q}\left(x_{1}, x_{2}, x_{3}\right) \quad \text { if } f\left(x_{1}\right)=f\left(x_{2}\right)=f\left(x_{3}\right)=q, \\ x_{1} & \text { if } f\left(x_{1}\right) \neq f\left(x_{2}\right), \\ x_{3} & \text { if } f\left(x_{1}\right)=f\left(x_{2}\right) \neq f\left(x_{3}\right) .\end{cases}
$$

Then $m_{f}\left(x_{1}, x_{2}, x_{3}\right) \in B_{f}$, by Theorem 7.7. But by Corollary 7.4 $\mathbf{F}_{\mathscr{V}}(3) \cong \prod_{f \in F} \mathbf{B}_{f}$ where $\mathscr{F}$ is some set of maps $f: X \rightarrow Q,\left[f\left(X^{\prime}\right)\right]=\mathbf{Q}, \mathbf{Q} \in \mathscr{V}_{F S}$. Then

$$
m(x, y, z)=\left\langle m_{f}(x, y, z): f \in \mathscr{F}\right\rangle
$$

is a Pixley term for $\mathscr{V}$, and hence $\mathscr{V}$ is arithmetical.

Corollary 8.3. Suppose $\mathscr{V}$ is a finitely generated variety. If all of the end nodes of $T(\mathscr{V})$ are arithmetical, then so is $\mathscr{V}$.

Proof. By induction on the height of $T(\mathscr{V})$ and the previous lemma.

In particular we have

COROLlaRY 8.4. Any finitely generated variety which has FHAP hereditarily is arithmetical.

We have not been able to give an intrinsic characterization of the varieties which have FHAP hereditarily, but we can identify a large class of them. First we establish some preparatory results.

LEMMA 8.5. Let $\mathscr{V}$ be a finitely generated arithmetical variety with FHAP. If $\mathscr{W} \in d(\mathscr{V})$, then $\mathscr{W}$ is arithmetical as well.

Proof. Let $m(x, y, z)$ be a Pixley term for $\mathscr{V}$. Suppose $\mathscr{W}=d(\mathscr{V})_{q}, q \in Q$, $\mathbf{Q} \in \mathscr{V}_{F S}$. Since $m(q, q, q)=q, m \in \tau_{q}$, and hence $\mathscr{W}$ has a Pixley term, too. Thus $\mathscr{W}$ is arithmetical.

By Theorem 5.1, an arithmetical variety has FHAP if all its subdirectly irreducible algebras have the Apple Property. In Example $1.2 \mathscr{B}_{k}$ meets this requirement, and so do the derived varieties, since they are of the same kind: $d\left(\mathscr{B}_{k}\right)=\left\langle\mathscr{B}_{0}, \mathscr{B}_{k-1}\right\rangle$. $\mathscr{B}_{k}$ is an example of what are called congruence relative Stone varieties with permuting congruences in Blok, Köhler and Pigozzi [1984]. A congruence relative Stone variety is any variety with equationally definable principal congruences 
(EDPC) whose subdirectly irreducibles have linearly ordered congruence lattices. A variety $\mathscr{V}$ is a congruence relative Stone variety of length $n(n<\omega)$ if $\operatorname{Co}(\mathbf{A})$ has length $\leqslant n$, for all $\mathbf{A} \in \mathscr{V}_{S I}$. Recall that any variety with EDPC is congruence distributive and that a sufficient condition for a congruence distributive variety to have EDPC is that it is locally finite, and that it has the congruence extension property. A quaternary function on an algebra $\mathbf{A}$ is called a normal transform on $\mathbf{A}$ if for all $a, b, c, d \in A$

$$
t(a, b, c, d)= \begin{cases}d & \text { if } c \equiv d \theta(a, b) \\ c & \text { otherwise. }\end{cases}
$$

Observe that the normal transform completely determines the congruence lattice of A. We recall the following theorem (Blok, Köhler and Pigozzi [1984, Theorem 4.3]).

THEOREM 8.6. Let $\mathscr{V}$ be a variety. The following are equivalent.

(i) $\mathscr{V}$ is a congruence relative Stone variety (of length $\leqslant n$ ) with permuting congruences.

(ii) There is a quaternary term $t$ of $\mathscr{V}$ such that for every subdirectly irreducible $\mathbf{A} \in \mathscr{V}, \operatorname{Co}(\mathbf{A})$ is a chain (of length $\leqslant n$ ) and $t$ coincides with the normal transform on $\mathbf{A}$.

The following lemma will be needed.

LEMmA 8.7. Let $\mathbf{A}$ be an algebra, such that $\mathrm{Co}(\mathbf{A})$ is a chain and $t$ a quaternary term which coincides with the normal transform on $\mathbf{A}$.

(i) Let $\mathbf{B}$ be any subalgebra of a reduct of $\mathbf{A}$ such that $t$ is a term for $\mathbf{B}$. Then $t$ coincides with the normal transform on $\mathbf{B}$, and $\mathrm{Co}(\mathbf{B})$ is a chain as well.

(ii) If $\mathbf{B} \in H(\mathbf{A})$, then $t$ coincides with the normal transform on $\mathbf{B}$, too.

Proof. (i) Let $a, b, c, d \in B$. If $c \equiv d \theta_{\mathbf{B}}(a, b)$, then certainly $c \equiv d \theta_{\mathbf{A}}(a, b)$, and hence $t(a, b, c, d)=d$. Since, conversely, $t(a, b, c, d)=d$ implies

$$
d=t(a, b, c, d) \equiv t(a, a, c, d)=c \quad \bmod \theta_{\mathbf{B}}(a, b),
$$

we get $t(a, b, c, d)=c$ if $c \not \equiv d \theta_{\mathbf{B}}(a, b)$. Thus $t$ coincides with the normal transform on $\mathbf{B}$. The last remark follows since $\theta_{\mathbf{A}}(a, b) \cap B^{2}=\theta_{\mathbf{B}}(a, b)$.

(ii) Let $\mathbf{B}=\mathbf{A} / \varphi, \varphi \in \operatorname{Co}(\mathbf{A})$, and $a, b, c, d \in A$. Then $c / \varphi \equiv d / \varphi, \theta(a / \varphi, b / \varphi)$ implies $c \equiv d \theta(a, b) \vee \varphi$. Since $\operatorname{Co}(\mathbf{A})$ is a chain, $\theta(a, b) \vee \varphi=\theta(a, b)$ or $\theta(a, b)$ $\vee \varphi=\varphi$. In the first case we have $t(a, b, c, d)=d$, and hence $t(a / \varphi, b / \varphi, c / \varphi, d / \varphi)=d / \varphi$. In the second case we get $c / \varphi=d / \varphi$, hence $t(a / \varphi, b / \varphi, c / \varphi, d / \varphi)=d / \varphi$ as well. If $c / \varphi \neq d / \varphi, \theta(a / \varphi, b / \varphi)$, then $c \neq \equiv d$, $\theta(a, b)$, and hence $t(a, b, c, d)=c$. Thus $t(a / \varphi, b / \varphi, c / \varphi, d / \varphi)=c / \varphi$, and $t$ coincides with the normal transform on $\mathbf{B}$.

We are now ready to state the main result of this section.

THEOREM 8.8. Let $\mathscr{V}$ be a finitely generated congruence relative Stone variety with permuting congruences. Then $\mathscr{V}$ has FHAP hereditarily. Furthermore, if $\mathscr{V}$ is of length $\leqslant n$, then $T(\mathscr{V})$ is of height $\leqslant n$. 
Proof. Since $\mathscr{V}$ is finitely generated, $\mathscr{V}$ is of length $\leqslant n$ for some $n<\omega$. By Theorems 8.6 and 5.1 any congruence relative Stone variety with permuting congruences has FHAP. In order to prove $\mathscr{V}$ has FHAP hereditarily, it is sufficient to show that every $\mathscr{W} \in T(\mathscr{V})$ is finitely generated, congruence relative Stone, and congruence permutable. We will do this by induction on the height of $\mathscr{W}$. If $h(\mathscr{W})=0$, then $\mathscr{W}=\mathscr{V}$ and by the hypotheses on $\mathscr{V}$ we are done. Suppose $\mathscr{W}^{\prime} \in T(\mathscr{V}), h\left(\mathscr{W}^{\prime}\right)>0$. Then $\mathscr{W}^{\prime}=d(\mathscr{W})_{q}$ for some $q \in Q, \mathbf{Q} \in \mathscr{W}_{F S}$ and we may assume $\mathscr{W}$ is finitely generated, congruence relative Stone, and congruence permutable. By 6.3(iii) $\mathscr{W}^{\prime}$ is finitely generated. Let $t$ be a quaternary term of $\mathscr{W}$ such that $t$ coincides with the normal transform on all subdirectly irreducible algebras in $\mathscr{W}$. Since $t(q, q, q, q)=q, t \in \tau_{q}$, and hence $t$ is a $d(\mathscr{W})_{q}$-term. We claim that $t$ coincides with the normal transform on all subdirectly irreducibles in $d(\mathscr{W})_{q}$ as well, and that $\operatorname{Co}(\mathbf{B})$ is a chain, for all $\mathbf{B} \in\left(d(\mathscr{W})_{q}\right)_{F S I}$. By Theorem 6.3(i), $d(\mathscr{W})_{q}=H S P\left\{\mathbf{A}_{q} \mid \mathbf{A} \in \mathscr{W}_{F S I}\right\}$. Since $\mathscr{W}$ is finitely generated, the set $\left\{\mathbf{A}_{q} \mid \mathbf{A}\right.$ $\left.\in \mathscr{W}_{F S I}\right\}$ is finite. By Lemma $8.5 d(\mathscr{W})_{q}$ is congruence distributive, and therefore $\left(d(\mathscr{W})_{q}\right)_{S I} \subseteq H S\left\{\mathbf{A}_{q}: \mathbf{A} \in \mathscr{W}_{F S I}\right\}$. It follows from Lemma 8.7(i) that $t$ coincides with the normal transform on the $\mathbf{A}_{q}$, and that $\operatorname{Co}\left(\mathbf{A}_{q}\right)$ is a chain, for all $\mathbf{A} \in \mathscr{W}_{F S I}$. Applying (i) and (ii) of the same Lemma 8.7, we see that $t$ coincides with the normal transform on all algebras in $\left(d(\mathscr{W})_{q}\right)_{S I}$, and that all of them have a congruence lattice which is linearly ordered. Thus by Theorem $8.6, d(\mathscr{W})_{q}$ is a congruence relative Stone variety with permuting congruences. Finally, if $\mathscr{W}$ is of length $\leqslant k$, then $\operatorname{Co}(\mathbf{A})$ is of length $\leqslant k$, for all $\mathbf{A} \in \mathscr{W}_{F S I}$. Let $\alpha$ be the coatom of $\operatorname{Co}(\mathbf{A})$. The map

$$
\theta_{\mathbf{A}_{q}}(a, b) \mapsto \theta_{\mathbf{A}}(a, b), \quad a, b \in A_{q},
$$

defines an embedding of $\operatorname{Co}\left(\mathbf{A}_{q}\right)$ into $(\alpha] \subseteq \operatorname{Co}(\mathbf{A})$. Thus $\operatorname{Co}\left(\mathbf{A}_{q}\right)$ is of length $\leqslant k-1, \mathbf{A} \in \mathscr{W}_{F S I}$, and hence so is $\operatorname{Co}(\mathbf{B})$, for all $\mathbf{B} \in\left(\mathscr{W}_{q}\right)_{S I}$. Thus $d(\mathscr{W})_{q}$ is of length $k-1$. It follows that no path of $T(\mathscr{V})$ can have length exceeding $n$, and thus that $T(\mathscr{V})$ has height $\leqslant n$.

The condition of the theorem is not necessary: there are finitely generated varieties (with CEP) which have FHAP, hereditarily but fail to be congruence relative Stone.

EXAMPLE 8.9. Let $A=\{0,1,2,3,4,5\}$, and let $\alpha, \beta, \gamma, \mu$ be equivalence relations on $A$ with partitions $P_{\alpha}=01\left|2345, P_{\beta}=01\right| 23\left|45, P_{\gamma}=01\right| 24 \mid 35$, and $P_{\mu}=$ $01|2| 3|4| 5$. Let $\mathbf{L}$ be the lattice $\{\Delta, \mu, \beta, \gamma, \alpha, \nabla\}$ in Figure 2. Then $\mathbf{L}$ is an arithmetical sublattice of the lattice of equivalence relations on $A$, and hence, by Pixley [1985, Lemma 3.1] there is a function $f: A^{3} \rightarrow A$, compatible with $\mathbf{L}$ such that for all $u, v, x, y \in A, f(u, v, v)=u, f(u, v, u)=u, f(u, u, v)=v$, and in addition, for any algebra whose congruence lattice is $\mathbf{L}$,

$$
x \equiv y \theta(u, v) \text { if and only if } f(u, v, x)=f(u, v, y) .
$$

Let $\mathbf{A}=\langle A, f, a\rangle_{a \in A}$. Then $\operatorname{Co}(\mathbf{A})=\mathbf{L}$, and $\mathscr{V}=H S P(\mathbf{A})$ is a finitely generated, arithmetical variety with CEP, but is clearly not congruence relative Stone. We will now show that $\mathscr{V}$ has FHAP hereditarily. Note that $\mathscr{V}_{S I}=I\{\mathbf{A}, \mathbf{A} / \beta, \mathbf{A} / \gamma, \mathbf{A} / \alpha\}$. 


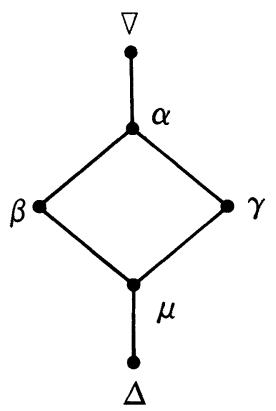

By Theorem 5.1, then, $\mathscr{V}$ has FHAP. Further, $\mathscr{V}_{F S}=I(\mathbf{A} / \alpha)$, and $\mathbf{A} / \alpha$ is polynomially equivalent to the 2-element Boolean algebra on $\{0,1\}$. By applying Theorem 6.3(iii) we see that $d(\mathscr{V})_{0}$ is polynomially equivalent to the variety of Boolean algebras. Similarly, $d(\mathscr{V})_{1}$ is the variety generated by the 4-element algebra $\mathbf{A}_{1}=$ $\langle\{2,3,4,5\}, f, i\rangle_{2 \leqslant i \leqslant 5}$, and hence polynomially equivalent to a variety of Boolean algebras with two extra constants. Thus $d(\mathscr{V})_{0}$ and $d(\mathscr{V})_{1}$ have FHAP, and since both are semisimple, we can conclude that $\mathscr{V}$ has FHAP hereditarily.

We conclude with some examples, illustrating the results and methods of this section.

EXAMPLE 8.10. Let $S_{2 n}=\{-n, \ldots,-2,-1,1,2, \ldots, n\}$, and let $S_{2 n+1}=S_{2 n} \cup\{0\}$, for $1 \leqslant n<\omega$, and $S_{1}=\{0\}$. Let $\mathbf{S}_{k}=\left\langle S_{k}, \vee, \wedge, \neg, \rightarrow\right\rangle, 1 \leqslant k<\omega$, where $x \vee$ $y=\max \{x, y\}, x \wedge y=\min \{x, y\}, \neg x=-x$, and

$$
x \rightarrow y= \begin{cases}\neg x \vee y & \text { if } x \leqslant y, \\ \neg x \wedge y & \text { if } x>y,\end{cases}
$$

and let $\mathscr{S}_{k}=\operatorname{HSP}\left(\mathbf{S}_{k}\right), k=1,2, \ldots$

The algebras in $\mathscr{S}_{k}$ are the Sugihara algebras of order $k, k<\omega$. They are the algebras associated with certain relevance logics, cf. Dunn [1970], Tokarz [1980], and Blok and Dziobiak [1985].

Since $\mathbf{S}_{k}$ has a lattice reduct, $\mathscr{S}_{k}$ is congruence distributive, and hence $\left(\mathscr{S}_{k}\right)_{S I} \subseteq$ $H S\left(\mathbf{S}_{k}\right)$. Note that $S\left(\mathbf{S}_{2 n}\right)=\left\{\mathbf{S}_{2 k}: 1 \leqslant k \leqslant n\right\}, S\left(\mathbf{S}_{2 n+1}\right)=\left\{\mathbf{S}_{k}: 1 \leqslant k \leqslant 2 n+1\right\}$. For $a, b \in S_{k},|a|<|b|$,

$$
x \equiv y \theta(a, b) \quad \text { iff } \quad x=y \text { or }-|b| \leqslant x, y \leqslant|b|,
$$

hence $\operatorname{Co}\left(\mathbf{S}_{2 n}\right) \cong \operatorname{Co}\left(\mathbf{S}_{2 n+1}\right) \cong \mathbf{n}+\mathbf{1}$, the $(n+1)$-element chain. We find that $H S\left(\mathbf{S}_{k}\right)=I\left\{\mathbf{S}_{j}: 1 \leqslant j \leqslant k\right.$, and thus $\left(\mathscr{S}_{k}\right)_{S I}=I\left\{\mathbf{S}_{j}: 2 \leqslant j \leqslant k\right\}$. It is easy to verify that the algebras $\mathbf{S}_{j}$ have the CEP, and hence, by Davey [1977], so does $\mathscr{S}_{k}$. Thus $\mathscr{S}_{k}$ is a congruence relative Stone variety. Finally,

$$
p(x, y, z)=[x \vee((z \rightarrow y) \rightarrow x)] \wedge[y \vee((x \rightarrow y) \rightarrow z)]
$$

is a $\mathrm{Mal}$ 'cev term for $\mathbf{S}_{k}$, hence $\mathscr{S}_{k}$ is congruence permutable as well. By Theorem $8.8, \mathscr{S}_{k}$ has FHAP hereditarily, and its height is $\left.\mid k\right\rceil / 2$.

We could have obtained this result in a more direct manner using Theorem 5.2. Let $k>2$ and $\mathscr{K}=\left\{\mathbf{S}_{j}: 2 \leqslant j \leqslant k\right\}$. Clearly $\mathbf{F}_{\mathscr{V}}(n) \in P_{S D}(\mathscr{K})$. The simples in 
$H(\mathscr{K})$ are isomorphic to $\mathbf{S}_{2}$ or $\mathbf{S}_{3}$, and since $\mathbf{S}_{3}$ is quasiprimal (cf. Berman [1983, \#086\#]), it therefore follows that $(H(\mathscr{K}))_{S}$ is multiplicative. Since $\operatorname{Co}\left(\mathbf{S}_{j}\right)$ is a finite chain, it has a unique coatom $\alpha$. Finally, by choosing $0_{S_{j}}, 1_{S_{i}},+, \cdot,^{\prime}$ to be $-j$, $+j, \vee, \wedge$, id, respectively, we see that (iv), (v), and (vi) of the hypotheses of Theorem 5.2 are satisfied as well. Thus the variety $\mathscr{S}_{k}$ has FHAP. Now let $\alpha$ be the coatom of $\operatorname{Co}\left(\mathbf{S}_{k}\right)$ and let $\mathbf{S}_{k} / \alpha=\mathbf{S}_{3}$. The $\alpha$-blocks of $\mathbf{S}_{k}$ are $\{-k\},[-k+1, k-1]$, and $\{k\}$, so that $\left(\mathbf{S}_{k}\right)_{-1}$ and $\left(\mathbf{S}_{k}\right)_{+1}$ are 1-element algebras, while $\left(\mathbf{S}_{k}\right)_{0}=\mathbf{S}_{k-2}$. Using Theorem 6.3 we infer $d\left(\mathscr{S}_{k}\right)_{-1}$ and $d\left(\mathscr{S}_{k}\right)_{+1}$ are trivial varieties, while $d\left(\mathscr{S}_{k}\right)_{0}=\mathscr{S}_{k-2}$. It thus follows again that $\mathscr{S}_{k}$ has FHAP hereditarily. For $k$ odd, $T\left(\mathscr{S}_{k}\right)$ looks like Diagram F.

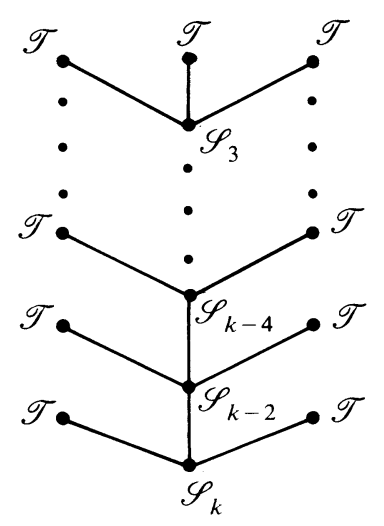

DIAGRAM F

Here $\mathscr{T}$ denotes the trivial variety.

Using the results of the previous section we can determine the structure and cardinality of the finitely generated free algebras in any of the $\mathscr{S}_{k}$. Of course, since $\mathscr{S}_{2}$ is the variety of Boolean algebras, $\left|\mathbf{F}_{\mathscr{S}_{2}}(n)\right|=2^{2^{n}}$ and $\mathbf{F}_{\mathscr{S}_{2}}(n) \cong \mathbf{S}_{2}^{2^{n}}$. The algebra $\mathbf{S}_{3}$ is \#086\# of Berman [1983]; $\left|\mathbf{F}_{\mathscr{S}_{3}}(n)\right|=2^{2^{n}} \cdot 3^{3^{n}-2^{n}-1}$ and $\mathbf{F}_{\mathscr{S}_{3}}(n) \cong \mathbf{S}_{2}^{2^{n}} \times$ $\mathbf{S}_{3}^{2^{n}-2^{n}-1}$.

First we discuss the free directly indecomposables associated with $\mathbf{S}_{3}$. Let $k>3$, and let for $l \geqslant 3, f_{l}: \mathbf{S}_{l} \rightarrow \mathbf{S}_{3}$ be the unique surjective homomorphism onto $\mathbf{S}_{3}$. We claim that Theorem 7.11 and Remark 7.13 apply. Note that $\left(\mathbf{S}_{k}\right)_{S I}=\left\{\mathbf{S}_{l}: 2 \leqslant l \leqslant k\right\}$. It is clear that for every $l, 2<l \leqslant k$, an embedding $j_{l}: \mathbf{S}_{l} \rightarrow \mathbf{S}_{k}$ can be found such that in fact $j_{l}:\left(\mathbf{S}_{l}, f_{l}\right) \rightarrow\left(\mathbf{S}_{k}, f_{k}\right)$. Therefore, given $X=\left\{x_{1}, \ldots, x_{n}\right\}, f: X \rightarrow S_{3}$ such that $[f(X)]=\mathbf{S}_{3}$, in applying Theorem 7.11 we can restrict ourselves to checking 7.12 for all $j:(X, f) \rightarrow\left(S_{k}, f_{k}\right)$. For example, if $f\left(x_{1}\right)=f\left(x_{2}\right)=0$, then for any fundamental operation $c\left(x_{1}, x_{2}\right)$ of $\mathbf{S}_{k}$ we can take $c_{0}=c$, as $\{0\}$ is a subalgebra of $\mathbf{S}_{3}$. If $f\left(x_{1}\right)=-1, f\left(x_{2}\right)=0$ then we can take $\neg_{1}\left(x_{1}\right)=x_{1} \vee \neg x_{1}$, $\vee_{0}\left(x_{1}, x_{2}\right)=x_{2}$, and $\rightarrow_{1}\left(x_{1}, x_{2}\right)=x_{1} \vee \neg x_{1}$. The various other cases can be treated similarly. It follows from the theorem that

$$
\left|F_{\left(\mathscr{S}_{k}\right)_{F D} \downarrow \mathrm{S}_{3}}(X, f)\right|=\sum_{i=-1}^{+1}\left|F_{d\left(\mathscr{S}_{k}\right)_{i}}\left(X_{i}\right)\right| .
$$


Since $d\left(\mathscr{S}_{k}\right)_{-1}=d\left(\mathscr{S}_{k}\right)_{+1}=\mathscr{T}$, and $d\left(\mathscr{S}_{k}\right)_{0}=\mathscr{S}_{k-2}$ we get

$$
\left|F_{\left(\mathscr{S}_{k}\right)_{F D} \downarrow \mathbf{S}_{3}}(X, f)\right|=\left|F_{\mathscr{S}_{k-2}}\left(\left|X_{0}\right|\right)\right|+2 \text {. }
$$

Actually, from the proof of Theorem 7.7 we can infer that

$$
\mathbf{F}_{\left(\mathscr{S}_{k}\right)_{F D I} \downarrow \mathbf{S}_{3}}(X, f)=\perp \oplus \mathbf{F}_{\mathscr{K}_{k-2}}\left(\left|X_{0}\right|\right) \oplus \top,
$$

where $\perp$ and $T$ are a new smallest and largest element, respectively, adjoined to $\mathbf{F}_{\mathscr{S}_{k-2}}\left(X_{0}\right)$ (cf. Blok and Dziobiak [1985]).

Since $\left(\mathscr{S}_{k}\right)_{F D I} \downarrow \mathbf{S}_{2}=\left\{\mathbf{S}_{2}\right\}$, the only free directly indecomposable associated with $\mathbf{S}_{2}$ is $\mathbf{S}_{2}$ itself. In order to find $\mathbf{F}_{\mathscr{S}_{k}}(X)$, we apply Corollary 7.5. Note that $\left(\mathscr{S}_{k}\right)_{0}=\mathscr{S}_{3}$, and as we observed already, $\mathbf{F}_{\mathscr{S}_{3}}(X)=\prod_{i=1}^{2^{n}} \mathbf{S}_{2} \times \prod_{i=2^{n}+1}^{3^{n}-1} \mathbf{S}_{3}$. For $1 \leqslant i$ $\leqslant 3^{n-1}$ let $f_{i}: X \rightarrow S_{3}$ be the map $\left.p_{i}\right|_{X}$. Then $\left\{f_{i}: 1 \leqslant i \leqslant 2^{n}\right\}=\{0,1\}^{X}$, and $\left\{f_{i}\right.$ : $\left.2^{n}+1 \leqslant i \leqslant 3^{n}-1\right\}$ is the set of all maps $f: X \rightarrow S_{3}$ such that $[f(X)]=\mathbf{S}_{3}$. Thus

$$
\mathbf{F}_{\left(\mathscr{S}_{k}\right)_{F D I} \downarrow \mathbf{S}_{2}}\left(X, f_{i}\right) \cong \mathbf{S}_{3}, \quad 1 \leqslant i \leqslant 2^{n},
$$

and

$$
\mathbf{F}_{\left(\mathscr{S}_{k}\right)_{F D I} \downarrow \mathbf{S}_{3}}\left(X, f_{i}\right) \cong \perp \oplus \mathbf{F}_{\mathscr{S}_{k-2}}\left(X_{0}\right) \oplus \mathrm{T}, \quad 2^{n}+1 \leqslant i \leqslant 3^{n}-1
$$

and hence

$$
\mathbf{F}_{\mathscr{S}_{k}}(n) \cong \mathbf{S}_{2}^{2^{n}} \times \prod_{\substack{n_{-1}+n_{0}+n_{1}=n \\
1 \leqslant n_{0} \leqslant n-1}}\left(\perp \oplus \mathbf{F}_{\mathscr{S}_{k-2}}\left(n_{0}\right) \oplus T\right)^{\left(\begin{array}{c}
n \\
n_{-1} n^{n_{1}}
\end{array}\right)}
$$

or, equivalently,

$$
\mathbf{F}_{\mathscr{S}_{k}}(n) \cong \mathbf{S}_{2}^{2^{n}} \times \prod_{n_{0}=1}^{n-1}\left(\perp \oplus \mathbf{F}_{\mathscr{S}_{k-2}}\left(n_{0}\right) \oplus \mathrm{T}\right)^{\left(n_{0}^{n}\right) 2^{n-n_{0}}} .
$$

Thus

$$
\left|F_{\mathscr{S}_{k}}(n)\right|=2^{2^{n}} \cdot \prod_{n_{0}=1}^{n-1}\left(\left|F_{\mathscr{S}_{k-2}}\left(n_{0}\right)\right|+2\right)^{\left(n_{0}^{n}\right) 2^{n-n_{0}}} .
$$

In a similar way it can be verified that Examples 1.2 and 1.4 have FHAP hereditarily, and recursive formulas for the structure and cardinality of their free objects can be found. Indeed, for $k \geqslant 2$, both $\mathscr{B}_{k}$ and $\mathscr{I}_{k}$ are congruence relative Stone with permuting congruences, of length $k$, and hence have FHAP hereditarily, by Theorem 8.8 . Up to isomorphism, the only simple algebra in $\mathscr{B}_{k}$ is the 2-element generalized Boolean algebra $\mathbf{Q}$ with 1 distinguished on $\{0,1\}$. Hence $\left(\mathscr{B}_{k}\right)_{0}$ is the variety of generalized Boolean algebras, and $\mathbf{F}_{\left(\mathscr{B}_{k}\right)_{0}}(n)=\mathbf{Q}^{2^{n}-1}$. More specifically, if $X=\left\{x_{1}, \ldots, x_{n}\right\}$, and $\mathscr{F}=\{f: X \rightarrow Q \mid[f(X)]=\mathbf{Q}\}$, then $\mathbf{F}_{\left(\mathscr{B}_{k}\right)_{0}}(X)=\Pi_{f \in F} \mathbf{Q}$. As in the Sugihara case, we can apply Theorem 7.11 and Remark 7.13 to determine the free directly indecomposables, and find that for $f \in \mathscr{F}$

$$
\mathbf{F}_{\left(\mathscr{B}_{k}\right)_{F D \downarrow} \downarrow \mathbf{Q}}(X, f)=\perp \oplus \mathbf{F}_{\mathscr{B}_{k-1}}\left(\left|X_{1}\right|\right)
$$


Here $X_{1}=f^{-1}(\{1\})$, and $\perp$ is a new smallest element. By Corollary 7.5

$$
\begin{aligned}
\mathbf{F}_{\mathscr{B}_{k}}(X) & \cong \prod_{f \in \mathscr{T}}\left(\perp \oplus \mathbf{F}_{\mathscr{B}_{k-1}}\left(f^{-1}(\{1\})\right)\right) \\
& \cong \prod_{0 \leqslant i \leqslant n-1}\left(\perp \oplus \mathbf{F}_{\mathscr{B}_{k-1}}(i)\right)^{\left(\begin{array}{l}
n \\
i
\end{array}\right)}
\end{aligned}
$$

(cf. Köhler [1973]).

Up to isomorphism, the only simple algebra in $\mathscr{I}_{k}$ is the 2-element interior algebra $\mathbf{I}_{2}$, with universe $\{0,1\}$. Hence the prime variety of $\mathscr{I}_{k},\left(\mathscr{I}_{k}\right)_{0}$, is $\mathscr{I}_{2}$. Since $\square 0=0, \square 1=1, \mathbf{I}_{2}$ is essentially a Boolean algebra, and $\mathbf{F}_{\mathscr{J}_{2}}(X)=\prod_{f \in\{0,1\}} \mathbf{I}_{2}$. As in Example 7.18 we can argue that $d\left(\mathscr{I}_{k}\right)_{1}=\mathscr{I}_{k-1}^{-}$, the variety of generalized interior algebras with 1 , generated by $\mathbf{I}_{k-1}^{-}$, while $d\left(\mathscr{I}_{k}\right)_{0}=\tilde{\mathscr{I}}_{k-1}^{-}$, the dual variety of $\mathscr{I}_{k-1}^{-}$, consisting of generalized closure algebras with 0 , and generated by $\tilde{\mathbf{I}}_{k-1}^{-}$. Corollary 7.17 applies again, hence, for $f \in\{0,1\}^{X}$,

$$
\left|\mathbf{F}_{\left(\mathscr{I}_{k}\right)_{F D I} \downarrow \mathbf{I}_{2}}(X, f)\right|=\left|\mathbf{F}_{\mathscr{I}^{-}-1}(X)\right|+\left|\mathbf{F}_{\tilde{\mathscr{F}}^{-}-1}(X)\right|=2 \cdot\left|\mathbf{F}_{\mathscr{F}_{k-1}^{-}}(X)\right|,
$$

and $\left|\mathbf{F}_{\mathscr{F}_{k}}(n)\right|=\left(2 \cdot\left|\mathbf{F}_{\mathscr{F}^{-}-1}(n)\right|\right)^{2^{n}}$. By similar reasoning $\left|\mathbf{F}_{\mathscr{F}^{-}-1}(n)\right|$ can be expressed in terms of $\left|\mathbf{F}_{\mathscr{I}_{k-2}}(n)\right|$ and continuing this way we obtain the cardinality of $\mathbf{F}_{\mathscr{f}_{k}}(n)$. The formula given in Example 1.4 reveals in addition the structure of $\mathbf{F}_{\mathscr{J}_{k}}(n)$; this goes beyond the methods of this paper and we refer the interested reader to Blok [1976] for details.

TABLE 1

Summary of results for some varieties $\mathscr{V}$ generated by a 3 -element algebra. The prime variety $\mathscr{V}_{0}$ contains a single simple algebra $\mathbf{Q}$ with universe $\{0,1\}$. The notation \#xyz refers to the entry number

\begin{tabular}{|c|c|c|c|c|c|}
\hline $\mathscr{V}$ & $\mathscr{V}_{0}$ & $\mathscr{U}=d(\mathscr{V})_{0}$ & $\mathscr{W}=d(\mathscr{V})_{1}$ & $\left|\mathbf{F}_{\mathscr{V}_{F D I} \downarrow \mathbf{Q}}(n, f)\right|$ & $\left|\mathbf{F}_{\mathscr{r}}(n)\right|$ \\
\hline $\begin{array}{l}\text { Stone alg. } \\
\text { Example } 1.1 \\
\# 186 \#\end{array}$ & $\begin{array}{l}\text { B. Alg. } \\
2^{n} \text { factors }\end{array}$ & $\begin{array}{l}\text { Trivial } \\
\left|\mathbf{F}_{\mathscr{U}}(i)\right|=1\end{array}$ & $\begin{array}{l}\text { Dist. lattices } \\
\text { with unit } \\
\mathbf{F}_{\mathscr{W}}(j)=\mathbf{F}_{\mathscr{D}_{1}}(j)\end{array}$ & $1+\left|\mathbf{F}_{\mathscr{D}_{1}}(j)\right|$ & $\prod_{j=0}^{n}\left(1+\left|\mathbf{F}_{\mathscr{D}_{1}}(j)\right|\right)^{\left(\begin{array}{c}n \\
j\end{array}\right)}$ \\
\hline $\begin{array}{l}\mathscr{B}_{3} \\
\text { Example } 1.2 \\
\# 103 \#\end{array}$ & $\begin{array}{l}\text { Gen. B. Alg. } \\
2^{n}-1 \text { factors }\end{array}$ & $\begin{array}{l}\text { Trivial } \\
\left|\mathbf{F}_{\mathscr{U}}(i)\right|=1\end{array}$ & $\begin{array}{l}\text { Gen. B. Alg. } \\
\left|\mathbf{F}_{\mathscr{W}}(j)\right|=2^{2^{j}-1}\end{array}$ & $1+2^{2^{j}-1}$ & $\prod_{j=0}^{n-1}\left(1+2^{2^{j}-1}\right)^{\left(\begin{array}{c}n \\
j\end{array}\right)}$ \\
\hline $\begin{array}{l}\text { Example } 1.3 \\
\text { with } \alpha=12 / 3 \\
\# 229 \#\end{array}$ & $\begin{array}{l}\text { B. Alg. } \\
2^{n} \text { factors }\end{array}$ & $\begin{array}{l}\text { B. Alg. } \\
\left|\mathbf{F}_{\mathscr{U}}(i)\right|=2^{2^{i}}\end{array}$ & $\begin{array}{l}\text { Trivial } \\
\left|\mathbf{F}_{\mathscr{W}}(j)\right|=1\end{array}$ & $1+2^{2^{i}}$ & $\prod_{i=0}^{n}\left(1+2^{2^{i}}\right)^{\left(\begin{array}{c}n \\
i\end{array}\right)}$ \\
\hline $\begin{array}{l}\text { Raca [1969] } \\
\text { Example 6.5 } \\
\# 185 \#\end{array}$ & $\begin{array}{l}\text { B. Alg. } \\
2^{n} \text { factors }\end{array}$ & $\begin{array}{l}\text { Trivial } \\
\left|\mathbf{F}_{\mathscr{U}}(i)\right|=1\end{array}$ & $\begin{array}{l}\text { Boolean groups } \\
\left|\mathbf{F}_{\mathscr{W}}(j)\right|=2^{j}\end{array}$ & $1+2^{j}$ & ]$_{0}\left(1+2^{j}\right)^{\left(\begin{array}{l}n \\
j\end{array}\right)}$ \\
\hline Example 6.6 & $\begin{array}{l}\text { B. Alg. } \\
2^{n} \text { factors }\end{array}$ & $\begin{array}{l}\text { Trivial } \\
\left|\mathbf{F}_{\mathscr{U}}(i)\right|=1\end{array}$ & $\begin{array}{l}\text { Bounded semilattices } \\
\left|\mathbf{F}_{\mathscr{W}}(j)\right|=1+2^{j}\end{array}$ & $1+1+2^{j}$ & $\prod_{j=0}\left(2+2^{j}\right)^{\left(\begin{array}{l}n \\
j\end{array}\right)}$ \\
\hline $\begin{array}{l}\text { Raca [1969] } \\
\text { Example } 6.8 \\
\# 130 \#\end{array}$ & $\begin{array}{l}\text { B. Rings } \\
2^{n}-1 \text { factors }\end{array}$ & $\begin{array}{l}\text { Trivial } \\
\left|\mathbf{F}_{\mathscr{Z}}(i)\right|=1\end{array}$ & $\begin{array}{l}\text { B. Rings } \\
\left|\mathbf{F}_{\mathscr{W}}(j)\right|=2^{2^{j}-1}\end{array}$ & $1+2^{2^{\prime}-1}$ & $\prod_{j=1}^{n}\left(1+2^{2^{j}-1}\right)^{\left(\begin{array}{c}n \\
j\end{array}\right)}$ \\
\hline $\begin{array}{l}\text { Heyting alg. } \\
\# 187\end{array}$ & $\begin{array}{l}\text { B. Alg. } \\
2^{n} \text { factors }\end{array}$ & $\begin{array}{l}\text { Trivial } \\
\left|\mathbf{F}_{\mathscr{U}}(i)\right|=1\end{array}$ & $\begin{array}{l}\text { Gen. B. Alg. } \\
\left|\mathbf{F}_{\mathscr{W}}(j)\right|=2^{2^{j}-1}\end{array}$ & $1+2^{2^{j}-1}$ & $\prod_{j=0}^{n}\left(1+2^{2^{j}-1}\right)^{\left(\begin{array}{c}n \\
j\end{array}\right)}$ \\
\hline
\end{tabular}
in Berman [1983]. The column with heading $\left|\mathbf{F}_{\mathscr{V}_{F D I} \downarrow \mathbf{Q}}(n, f)\right|$ is the cardinality of the free directly indecomposable with $n$ free generators, $i=\left|f^{-1}(0)\right|, j=\left|f^{-1}(1)\right|, n=i+j$. 
TABLE 2

Summary of results, for the example varieties. The column headed $\mathscr{V}_{0}$ gives a description of the simple algebras in $\mathscr{V}_{0}$ and the number of directly indecomposable factors of $\mathbf{F}_{\mathscr{V}_{0}}(n)$ (and also of $\mathbf{F}_{\mathscr{V}}(n)$ ). The column with heading $\left|\mathbf{F}_{\mathscr{V}_{F D I} \downarrow \mathbf{Q}}(n, f)\right|$ gives the cardinality of the free directly indecomposable with $n$ free generators, for $i \in Q, n_{i}=\left|f^{-1}(i)\right|, \sum n_{i}=n$.

\begin{tabular}{|c|c|c|c|c|}
\hline $\mathscr{V}$ & $\mathscr{V}_{0}$ & $d(\mathscr{V})$ & $\left|\mathbf{F}_{\mathscr{V}_{F D I} \downarrow \mathbf{Q}}(n, f)\right|$ & $\left|\mathbf{F}_{\mathscr{Y}}(n)\right|$ \\
\hline $\begin{array}{l}\mathscr{B}_{k} \\
\text { Brouwerian alg. } \\
\text { Example } 1.2 \\
\end{array}$ & $\begin{array}{l}\mathbf{Q} \text { is a gen. B. alg. } \\
Q=\{0,1\} \\
2^{n}-1 \text { factors }\end{array}$ & $\begin{array}{l}d(\mathscr{V})_{0} \text { is trivial } \\
d(\mathscr{V})_{1}=\mathscr{B}_{k-1}\end{array}$ & $1+\left|\mathbf{F}_{\mathscr{B}_{k-1}}\left(n_{1}\right)\right|$ & $\prod_{n_{1}=0}^{n-1}\left(1+\left|\mathbf{F}_{\mathscr{D}_{k-1}}\left(n_{1}\right)\right|\right)^{\left(n_{1} 1\right)}$ \\
\hline $\begin{array}{l}\mathscr{E} \\
\text { Example } 1.3\end{array}$ & $\begin{array}{l}\mathbf{Q} \text { is primal } \\
Q=\{1, \ldots, k\} \\
k^{n} \text { factors }\end{array}$ & $\begin{array}{l}d(\mathscr{V})_{i}=H S P\left(\mathbf{A}_{i}\right) \\
\mathbf{A}_{i} \text { is primal } \\
\left|A_{i}\right|=c_{i}\end{array}$ & $\begin{array}{l}c_{1}^{m}+\cdots+c_{k}^{m} \\
\text { where } m= \\
c_{1}^{n_{1}} c_{2}^{n_{2}} \cdots c_{k}^{n_{k}}\end{array}$ & $\begin{array}{l}\text { As displayed } \\
\text { in Example } 1.3\end{array}$ \\
\hline $\begin{array}{l}\mathscr{I}_{k} \\
\text { Interior alg. } \\
\text { Example } 1.4 \text {, Fact } 7.8\end{array}$ & $\begin{array}{l}\mathbf{Q} \text { is a } \mathbf{B} . \text { alg. } \\
\mathbf{Q}=\{0,1\} \\
2^{n} \text { factors }\end{array}$ & $\begin{array}{l}d(\mathscr{V})_{0}=\tilde{\mathscr{I}}_{k-1}^{-} \\
d(\mathscr{V})_{1}=\mathscr{I}_{k-1}^{-}\end{array}$ & $2\left|\mathbf{F}_{\mathscr{F}^{-}-1}(n)\right|$ & $\left(2\left|\mathbf{F}_{\mathscr{I}^{-}-1}(n)\right|\right)^{2^{n}}$ \\
\hline $\begin{array}{l}H S P\left(\mathbf{Z}_{p^{2}}\right) \\
\text { Examples 5.5, } 7.19\end{array}$ & $\begin{array}{l}Z_{p}=\{0,1, \ldots, p-1\} \\
p^{n} \text { factors }\end{array}$ & $\begin{array}{l}d(\mathscr{V})_{i}=\mathscr{A} b_{p}^{*} \\
i=0, \ldots, p-1\end{array}$ & $p^{n+2}$ & $\left(p^{n+2}\right)^{p^{n}}$ \\
\hline Example 6.7 & $\begin{array}{l}\mathbf{Q} \text { is primal } \\
Q=\{0,1, \ldots, m-1\} \\
m^{n} \text { factors }\end{array}$ & $d(\mathscr{V})_{i}=\operatorname{HSP}\left(\mathbf{A}_{i}\right)$ & $\sum_{i=1}^{m}\left|\mathbf{F}_{d(\mathscr{V})_{i}}\left(n_{i}\right)\right|$ & $\begin{array}{l}\text { As displayed } \\
\text { in }(7.14)\end{array}$ \\
\hline $\begin{array}{l}\text { Complemented } \\
\text { Semigroups } \\
\text { Example } 6.9\end{array}$ & $\begin{array}{l}\mathbf{Q}_{1}=\langle\{0,1\}, \cdot, *\rangle \\
\mathbf{Q}_{2}=\langle\{2,3\}, \cdot, *\rangle \\
\text { each has } \\
2^{n}-1 \text { factors }\end{array}$ & $\begin{array}{l}d(\mathscr{V})_{1}=\mathrm{B} . \text { rings } \\
d(\mathscr{V})_{i} \text { is trivial } \\
\text { ior } i=0,2,3\end{array}$ & $\begin{array}{l}\mathbf{Q}_{1}: 1+2^{2^{n_{1}}-1} \\
\mathbf{Q}_{2}: 2\end{array}$ & $2^{2^{n}-1} \prod_{n_{1}=0}^{n-1}\left(2^{2^{n_{1}}-1}+1\right)^{\left(n_{1}^{n}\right.}$ \\
\hline $\begin{array}{l}S_{k} \\
\text { Sugihara Alg. } \\
\text { Example } 8.10\end{array}$ & $\begin{array}{l}\mathbf{Q}_{1}=\mathbf{S}_{3} \\
Q_{1}=\{-1,0,1\} \\
3^{n}-2^{n}-1 \text { factors } \\
\mathbf{Q}_{2} \cong \mathbf{S}_{2} \\
Q_{2}=\{2,3\} \\
2^{n} \text { factors }\end{array}$ & $\begin{array}{l}d(\mathscr{V})_{0}=\mathscr{S}_{k-2} \\
d(\mathscr{V})_{i} \text { is trivial } \\
\text { for } i=-1,1,2,3\end{array}$ & $\begin{array}{l}\mathbf{Q}_{1}: \\
\left|\mathbf{F}_{\mathscr{S}_{k-2}}\left(n_{0}\right)\right|+2 \\
\mathbf{Q}_{2}: 2\end{array}$ & $\begin{array}{l}\text { As displayed } \\
\text { in }(8.11)\end{array}$ \\
\hline
\end{tabular}

\section{REFERENCES}

R. Balbes and Ph. Dwinger [1974], Distributive lattices, Univ. of Missouri Press.

R. Balbes and A. Horn [1970], Stone lattices, Duke Math. J. 37, 537-546.

J. Berman [1983], Free spectra of 3-element algebras, Universal Algebra and Lattice Theory, Proceedings 1982, Lecture Notes in Math., vol. 1004, Springer-Verlag.

W. J. Blok [1976], Varieties of interior algebras, Dissertation, Univ. of Amsterdam.

W. J. Blok and W. Dziobiak [1985], On the lattice of quasivarieties of Sugihara algebras, Studia Logica (to appear).

W. J. Blok, P. Köhler and D. Pigozzi [1984], On the structure of varieties with equationally definable principal congruences. II, Algebra Universalis 18, 334-379.

B. Bosbach [1969], Komplementare Halbgruppen, Fund. Math. 64, 257-287.

S. Burris [1982], Discriminator polynomials and arithmetical varieties, manuscript.

S. Burris and J. Lawrence [1981], A correction to "Definable principal congruences in varieties of groups and rings," Algebra Universalis 13, 264-267.

S. Burris and H. Sankappanavar [1981], A course in universal algebra, Graduate Texts in Math., No. 78, Springer-Verlag.

W. H. Cornish [1983], Antimorphic action, preprint, Flinders Univ., 186 pp.

B. A. Davey [1977], Weak injectivity and congruence extension in congruence-distributive equational classes, Canad. J. Math. 29, 449-459.

J. Demetrovics, L. Hannak and L. Ronyai [1982], On the free spectra of maximal clones, C. R. Math. Rep. Acad. Sci. Canada 4, 363-366. 
J. M. Dunn [1970], Algebraic completeness results for R-mingle and its extensions, J. Symbolic Logic 35 , $1-13$.

A. L. Foster and A. F. Pixley [1964], Semicategorical algebras, II, Math. Z. 85, 169-184.

G. A. Fraser and A. Horn [1970], Congruence relations in direct products, Proc. Amer. Math. Soc. 26, 390-394.

R. S. Freese and J. B. Nation [1973], Congruence lattices of semilattices, Pacific J. Math. 49, 51-58.

G. Gratzer [1979], Universal algebra, 2nd ed., Springer-Verlag.

G. Gratzer and E. T. Schmidt [1957], On a problem of M. H. Stone, Acta Math. Acad. Sci. Hungar. 8, $455-460$.

A. Horn [1969], Free L-algebras, J. Symbolic Logic 34, 475-480.

T. K. Hu [1970], On equational classes of algebras in which congruences on finite products are induced by congruences on their factors, manuscript.

B. Jónsson [1967], Algebras whose congruence lattices are distributive, Math. Scand. 21, 110-121.

P. Köhler [1973], Freie endlich erzeugte Heyting Algebren, Diplomarbeit, Justus Liebig Universität, Giessen.

H. Lakser [1982], Principal congruences in N-permutable varieties, Algebra Universalis 14, 64-67.

S. Mac Lane [1971], Categories for the working mathematician, Springer-Verlag.

R. McKenzie [1982], Narrowness implies uniformity, Algebra Universalis 15, 67-85. [1984], A new product of algebras and a type reduction theorem, Algebra Universalis 18, 29-69.

R. McKenzie and D. Hobby [1986], The structure of finite algebras (tame congruence theory), manuscript.

A. F. Pixley [1971], The ternary discriminator function in universal algebra, Math. Ann. 191, 167-180. [1985], Principal congruence formulas in arithmetical varieties, Universal Algebra and Lattice Theory, Lecture Notes in Math., vol. 1149, Springer, pp. 238-254.

J. Plonka [1971], On free algebras and algebraic decomposition of algebras from some equational classes defined by regular equations, Algebra Universalis 1, 261-264.

E. L. Post [1921], Introduction to a general theory of elementary propositions, Amer. J. Math. 43, $163-185$.

R. W. Quackenbush [1974], Structure theory for equational classes generated by quasi-primal algebras, Trans. Amer. Math. Soc. 187, 127-145.

M. F. Raca [1969], The class of functions of the three-valued logic that corresponds to the first matrix of Jas'kovski, Problemy Kibernet. 21, 185-214. (Russian)

W. Taylor [1975], The fine spectrum of a variety, Algebra Universalis 5, 263-303.

M. Tokarz [1980], Essays in matrix semantics of relevant logics, Polish Acad Sci., Institute of Philosophy and Sociology, Warszawa.

H. Werner [1970], Eine Charakterisierung funktional vollständiger Algebren, Arch. Math. (Basel) 21, $381-385$.

Department of Mathematics, University of Illinois at Chicago, Box 4348, Chicago, Illinois 60680 